\title{
Boulesic-Doxastic Logic
}

\author{
Daniel Rönnedal \\ Department of Philosophy, Stockholm University \\ daniel.ronnedal@philosophy.su.se
}

\begin{abstract}
In this paper, I will develop a set of boulesic-doxastic tableau systems and prove that they are sound and complete. Boulesic-doxastic logic consists of two main parts: a boulesic part and a doxastic part. By 'boulesic logic' I mean 'the logic of the will', and by 'doxastic logic' I mean 'the logic of belief'. The first part deals with 'boulesic' concepts, expressions, sentences, arguments and theorems. I will concentrate on two types of boulesic expression: 'individual $x$ wants it to be the case that' and 'individual $x$ accepts that it is the case that'. The second part deals with 'doxastic' concepts, expressions, sentences, arguments and theorems. I will concentrate on two types of doxastic expression: 'individual $x$ believes that' and 'it is imaginable to individual $x$ that'. Boulesic-doxastic logic investigates how these concepts are related to each other. Boulesic logic is a new kind of logic. Doxastic logic has been around for a while, but the approach to this branch of logic in this paper is new. Each system is combined with modal logic with two kinds of modal operators for historical and absolute necessity and predicate logic with necessary identity and 'possibilist' quantifiers. I use a kind of possible world semantics to describe the systems semantically. I also sketch out how our basic language can be extended with propositional quantifiers. All the systems developed in this paper are new.
\end{abstract}

Keywords: Boulesic-doxastic logic, Boulesic logic, doxastic logic, modal logic, semantic tableaux, practical rationality.

\section{Introduction}

There are two important types of propositional attitudes: boulesic (from the Greek 'boulesis') and doxastic (from the Greek 'doxa'). Boulesic attitudes include attitudes such as wanting, willing, accepting, consenting, intending, desiring, rejecting, loving and hating, while doxastic attitudes include attitudes such as believing, holding true, conceiving and imagining. Boulesic logic deals with the former, dox- 
astic logic with the latter ${ }^{1}$, and boulesic-doxastic logic investigates both. In this paper, I will focus on two types of boulesic expression: 'individual $x$ wants it to be the case that' and 'individual $x$ accepts that it is the case that', and two types of doxastic expression: 'individual $x$ believes that' and 'it is imaginable to individual $x$ that'. Boulesic-doxastic logic deals with both boulesic and doxastic words, concepts, expressions, sentences, principles, arguments and systems, and with the relationships between these concepts, and so on.

In this paper, I will develop a set of boulesic-doxastic tableau systems ${ }^{2}$, I will investigate several interesting boulesic-doxastic principles and I will establish some important theorems. Each system is combined with modal logic with two kinds of modal operators for historical and absolute necessity, and predicate logic with necessary identity and 'possibilist' quantifiers. ${ }^{3}$ I also sketch out how our basic language can be extended with propositional quantifiers, and how we can define several interesting concepts that can be used to say something about what it might mean to be (perfectly) rational or wise, for example the concept of perfect self-awareness. ${ }^{4}$ All the systems developed in this paper are new. ${ }^{5}$ I will use a kind of possible world semantics to describe them semantically and I will prove that each (non-augmented) logic is sound and complete with respect to its semantics. A non-augmented logic does not include the propositional quantifiers.

The systems in this paper include four operators that can be used to represent various propositional attitudes: $\mathcal{W}, \mathcal{A}, \mathcal{B}$ and $\mathcal{C}$. Let $t$ be a term (that represents some individual) and let $D$ be any well-formed sentence. Then all of the following formulas are well-formed in our language: $\mathcal{W}_{t} D, \mathcal{A}_{t} D, \mathcal{B}_{t} D$ and $\mathcal{C}_{t} D$. ' $\mathcal{W}_{t} D$ ' says that individual $t$ wants it to be the case that $D,{ }^{\prime} \mathcal{A}_{t} D$ ' says that $t$ accepts that it

\footnotetext{
${ }^{1}$ For more on doxastic logic (and epistemic logic, which deals with the concept of knowledge) see, for example, Fagin, Halpern, Moses and Vardi (1995), Gochet and Gribomont (2006), Hintikka (1962), Meyer and van der Hoek (1995) and van Ditmarsch, van der Hoek and Kooi (2008).

${ }^{2}$ The kind of technique I use is inspired by, for example, Jeffrey (1967), Priest (2008) and Smullyan (1968). A general introduction to the tableau method can be found in D'Agostino, Gabbay, Hähnle and Posegga (1999). See also Fitting and Mendelsohn (1998).

${ }^{3}$ For various introductions to ordinary (alethic) modal logic, see, for example, Blackburn, de Rijke and Venema (2001), Blackburn, van Benthem, Wolter (2007), Chellas (1980), Fitting and Mendelsohn (1998), Gabbay (1976), Garson (2006), Kracht (1999) and Lewis and Langford (1932). For more on modal predicate logic see, for example, Barcan (Marcus) (1946), Carnap (1946), Garson (1984, 2006), Hughes and Cresswell (1968) and Priest (2008).

${ }^{4}$ For more on propositional quantifiers see, for example, Bull (1969), Fine (1970, 1980), Gallin (1975), Grover (1972), Kaplan (1970), Kripke (1959) and Lewis and Langford (1932, pp. 178-198), and for more on the concept of rationality, see Mele (2004).

${ }^{5}$ Boulesic logic is an entirely new kind of logic. However, for some vaguely similar approaches, see, for example, Broersen (2011), Broersen, Dastani and van der Torre (2001), Cohen and Levesque (1990), Gensler (2002), Chapter 10, Lorini and Herzig (2008), Marra and Klein (2015) and Semmling and Wansing (2008).
} 
is the case that $D$ (or that $t$ consents to the state of affairs that $D$ ), ' $\mathcal{B}_{t} D$ ' says that $t$ believes that it is the case that $D$ and ' $\mathcal{C}_{t} D$ ' says that it is (doxastically) conceivable to $t$ that $D$. Since $D$ is any well-formed sentence it is meaningful to speak about wanting anything, believing anything, etc. $D$ can be about the present time (I want to eat (now); I believe that it is raining (now)), about the future (Mary wants to go for a walk tomorrow; He believes that Germany will win the soccer tournament), or about the past (I want [hope, desire, wish] that I made a good impression yesterday ${ }^{6}$; I believe that I paid the bill last week); it can be about a contingent state of affairs (Harry wants to go to the movies this weekend; She believes that Paris is the capital of France) or a necessary state of affairs (He wants the Goldbach conjecture to be true; She believes that the law of non-contradiction is valid); it can be about facts concerning the "natural' world (She wants the weather to be sunny; He believes that water includes oxygen) or about the 'mental' world (She wants to believe that he is faithful to her; He believes that he wants to be faithful to her); it can be about $t$ (He wants to be smart; She believes that she is smart) or about some other individual or individuals (She wants Will to take out the trash; He believes that Sally believes that he is a doctor), it can be about things within $t$ 's control (He wants to go to the store; She believes that she will call him) or about things that are not within $t$ 's control (Fanny wants Peter to love her; Paul believes that the sun will rise tomorrow); it can be about something that involves $t$ 's own agency (He wants to go for a swim; She believes that she is thinking about philosophy) or about something that does not involve $t$ 's own agency (She wants to be famous; He believes that he is tall); and so on. If $t$ is not perfectly rational (reasonable or wise), it is even possible that $t$ wants or believes impossible states of affairs. A person who is not rational can both want and reject something at the same time according to our systems in this paper (He wants to be with her and he wants not to be with her.). It does not appear to be rational to have inconsistent propositional attitudes of this kind, but it is not logically impossible.

When we say that some individual $t$ wants something (or accepts something) $A$, we usually mean that $t$ wants (accepts) $A$ in an all-things-considered sense in this paper. For example, $t$ might not feel like going to the dentist, in fact, $t$ might very much dislike going to the dentist. Nevertheless, all-things-considered $t$ wants to go to the dentist. Going to the dentist is a means to an end, namely, healthy teeth. So, when we say that $t$ wants (or accepts) something, we do not necessarily mean that $t$ wants (or accepts) this 'thing' in itself. It is possible to want (and

\footnotetext{
${ }^{6}$ Some philosophers seem to think that all desires (and wants) are oriented towards the future (see, for example, Sumner, 1996, pp. 128-130 and Sumner, 2000), while other philosophers appear to reject this idea (see, for example, Fred Feldman, 2004, pp. 61-63). Most wants (desires) that people have seem to be directed at the future or the present and concern contingent states of affairs. Yet, we will not rule out the possibility of past-directed wants in this paper.
} 
accept) something as a means to something else and it is possible to want (and accept) something in itself. Furthermore, it is possible for $t$ to want (or accept) $A$ in an all-things-considered sense even if $t$ does not like every aspect of $A$ or every consequence of $A$ and even if $t$ has some desire (a prima facie desire) for not- $A$.

For a perfectly rational individual wanting is a 'stronger' attitude than consenting (at least, in every system that includes the tableau rule $T-b D$ (Table 13)). If a perfectly rational individual wants $A$, she also consents to $A$ (given that we accept condition $C-b D$ (Table 2)). However, it is possible for a perfectly rational individual to consent to something that she does not want. A perfectly rational individual may, for example, consent to not getting any help in a particular situation even though not getting any help is not something she wants. Instead of 'consent' or 'acceptance', we can sometimes use the words 'agree', 'allow', 'approve', 'condone' or 'tolerate'. As I have already mentioned, consenting to something usually means consenting to it all-things-considered in this paper. It is possible for someone to consent to $A$ even though she objects to some aspects of or consequences of $A$. In every system in this paper, it is possible (even for a perfectly rational individual) to consent to $A$ and (at the same time) to consent to not- $A$.

In the sense that I am using the terms in this paper, intentions and wants are not the same thing. It is possible to want someone else to do something, but it is not possible that you intend someone else to do something. Intentions are directed towards (our own) actions, while it is possible to want and desire and accept all sorts of things. Wanting to do something and intending to do it is perhaps the same thing, but it is not obvious that this is the case. Even if this were true (and I am not denying that it is true), wants would have a 'wider scope' than intentions. Wanting something is also not the same as wishing it were true. Wishing something impossible were true might be possible even for a perfectly rational individual, even though it seems to be reasonable to claim that no perfectly rational individual wants impossible things (in an all-things-considered sense).

Since all the systems in this paper are new, the results are technically valuable. There are also many good philosophical reasons to be interested in the systems in this paper. Space does not permit me to discuss every possible argument, but I will briefly mention some of the most obvious ones.

Reason 1. There are several problems with standard systems of doxastic logic that we can avoid in our systems, for example the problem of logical omniscience (see Meyer and van der Hoek (1995, pp. 71-89) for an introduction). According to this problem, the notions of knowledge and belief that are used in ordinary epistemic and doxastic symbolic systems are too strong; they are only reasonable for 'ideal' individuals. For example, the following rules of inference hold in most standard systems $\left(B_{i} A\right.$ reads 'individual $i$ believes that $A$ '): 
If $A \leftrightarrow C$ is a theorem, then $B_{i} A \leftrightarrow B_{i} C$ is a theorem (for every $i$ and $A$ and $C$ ) (Belief of equivalent formulas).

If $A \rightarrow C$ is a theorem, then $B_{i} A \rightarrow B_{i} C$ is a theorem (for every $i$ and $A$ and $C$ ) (Closure under valid implication).

If $A$ is a theorem, then $B_{i} A$ is a theorem (for every $i$ and $A$ ) (Belief of valid formulas).

If we want to use doxastic logic to describe ordinary human or human-like agents (non-human animals, computers, robots, etc.), then all these inference patterns are unreasonable. It is implausible to assume that an organism such as a human being can believe every valid sentence no matter how complex it is. It is also unreasonable to suppose that a human-like agent's beliefs are closed under valid implication. As far as we know, no human-like agent believes every logical consequence of what she believes no matter how complicated it is. Moreover, even 'Belief of equivalent formulas' is problematic. Suppose that $A$ and $B$ are logically equivalent. Does it follow that the agent believes $A$ iff she believes $B$ ? For some $A$ and some $B$ this is perhaps the case, but is the principle true for every $A$ and $B$ no matter how complicated these propositions are? The rules of inference above hold in standard doxastic systems due to the fact that doxastic (and epistemic) logic traditionally has been developed as a form of normal modal logic. The first rule (Belief of equivalent formulas) is a problem also for many non-normal systems. Many formulas that can be proved in (most) standard systems are also problematic. Consider the following examples:

$$
\begin{aligned}
& \left(B_{i} A \wedge B_{i}(A \rightarrow C)\right) \rightarrow B_{i} C . \\
& \neg\left(B_{i} A \wedge B_{i} \neg A\right) . \\
& B_{i}(A \wedge C) \leftrightarrow\left(B_{i} A \wedge B_{i} C\right) .
\end{aligned}
$$

In our systems, the rules of inference and the formulas above do not hold. Hence, we can solve the problem of logical omniscience. Almost nothing of interest follows from the fact that someone believes something.

Let us consider a more concrete example of a situation that is problematic for many standard systems of doxastic logic. The following scenario appears to be perfectly logically possible:

Example 1. (The Triangle Scenario) Mike believes that this figure is a triangle (because he can see that this figure has three edges and three vertices). Mike believes that if this figure is a triangle this figure is equiangular (because someone in the past told him that all triangles are equiangular). But it is not the case that he believes that this figure is equiangular, in fact, he believes that this figure is not 
equiangular (because he can see that the angles are not equal). Of course, what Mike believes is inconsistent. Hence, his beliefs are inconsistent. Accordingly, it is reasonable to conclude that he is not perfectly rational. But people sometimes have inconsistent beliefs and not everyone is perfectly rational. (It might even be the case that no-one is.)

Yet, according to normal systems of doxastic logic The Triangle Scenario does not describe a logically possible situation. Any set of the following form is inconsistent in most standard doxastic logics (and in every normal doxastic logic): $\left\{B_{i} A, B_{i}(A \rightarrow C), \neg B_{i} C\right\}$. In our systems, the propositions in The Triangle Scenario can be symbolised in the following way: $\mathcal{B}_{c} T t, \mathcal{B}_{c}(T t \rightarrow Q t), \neg \mathcal{B}_{c} Q t$ and $\mathcal{B}_{c} \neg Q t$, where ' $T$ ' stands for 'is a triangle', ' $Q$ ' stands for 'is equiangular', ' $c$ ' refers to Mike and ' $t$ ' refers to this figure. According to our semantics, it is possible that there is a possible world in which all these sentences are true. In Section 3.2 , I will verify this assertion. This example clearly shows that the systems in this paper are much more realistic than many other systems in the literature.

However, if the inference rules and the principles above are restricted to perfectly rational (reasonable or wise) individuals they might be reasonable. In every system that includes the tableau rule $T-d D$ (see Table 14 in Section 4.2.7) we can, for example, prove that no perfectly rational (reasonable or wise) individual has inconsistent beliefs; that is, we can prove the following formula: $\neg \Sigma x\left(R x \wedge\left(\mathcal{B}_{x} A \wedge \mathcal{B}_{x} \neg A\right)\right)$ (there is no $x$ such that $x$ is perfectly rational and $x$ believes that $A$ and $x$ believes that not $-A$ ).

It is logically possible that no-one has any inconsistent beliefs at some time. It might even be factually true that there is some moment in time when no-one has any inconsistent beliefs (if there is a time when there are no agents that believe anything, this proposition will be vacuously true). But even if this is possible, it appears to be implausible to assume that it is a logical truth that no-one has inconsistent beliefs. Not all truths are logical truths and we are not primarily interested in contingent truths in this paper.

Many different solutions to the problem of logical omniscience have been suggested in the literature. ${ }^{7}$ I will not try to discuss these solutions in the present paper. However, every solution seems to suffer from the same kind of problem: it is based on some system that is intuitively too strong, intuitively too weak or simultaneously both too strong and too weak. A system is too strong if it includes too many theorems, that is, if we can prove things in this system that are counterintuitive; and it is

\footnotetext{
${ }^{7}$ For more on this, see, for example, Fagin and Halpern (1988), Girle (1998), Gochet and Gillet (1991), Gochet and Gribomont (2006), Hocutt (1972), Jaspars (1991), Levesque (1984), McLane (1979), Rantala (1982), Sim (1997, 2000), Thijsse (1992), van der Hoek and Meyer (1989), Yap (2014).
}

Australasian Journal of Logic (16:3) 2019, Article no. 3 
too weak, if it contains too few theorems, that is, if we cannot prove all intuitively plausible principles that we want to establish. For example, classical systems appear to be too strong, since Belief of equivalent formulas (see above) still holds in such systems, and systems that use impossible worlds often seem to be too weak. It is intuitively reasonable to assume that a perfectly rational individual will not believe any contradiction. We cannot show this in systems based on some kind of 'standard' impossible world semantics. The systems in this paper are partly weaker and partly stronger than many standard systems. Some intuitively problematic sentences and arguments that can be established in standard systems are not valid in our systems, and some intuitively plausible sentences and arguments that are not valid in standard systems can be established in our systems. It follows that we can avoid many problems with classical doxastic logic and with many other solutions to the problem of logical omniscience. According to some solutions, we must postulate various new kinds of entities that might be ontologically problematic, for example impossible things or impossible worlds. Our semantics do not presuppose the existence (or being) of any entities of this kind. In conclusion, there are good reasons to be interested in the results in this paper.

Reason 2. The systems in this paper can be used to investigate many interesting principles, for example the principle that no-one who is perfectly rational or wise wants it to be the case that $A$ at the same time as she wants it to be the case that not- $A$, and the principle that no-one who is perfectly rational or wise believes that it is the case that $A$ at the same time as she believes that it is the case that not- $A$. In particular, we can investigate many interesting principles that include both boulesic and doxastic expressions. Consider the following examples:

\section{Example 2. (Some boulesic-doxastic principles)}

(i) If someone is perfectly rational (wise), then if she wants something she believes that she wants it; that is, she is aware of everything she wants.

(ii) If $x$ is perfectly rational (wise), then $x$ wants $A$ only if $x$ believes that it is possible that $A$.

(iii) If $x$ is perfectly rational (wise), then if $x$ wants it to be the case that $A$ and $x$ believes that it is necessary that if $A$ then $B$ then $x$ wants it to be the case that $B$.

To be able to study such principles we need boulesic-doxastic logic. (i)-(iii) can be symbolised in the following way in our systems: (i) $\Pi x\left(R x \rightarrow\left(\mathcal{W}_{x} A \rightarrow\right.\right.$ $\left.\left.\mathcal{B}_{x} \mathcal{W}_{x} A\right)\right)$, (ii) $\Pi x\left(R x \rightarrow\left(\mathcal{W}_{x} A \rightarrow \mathcal{B}_{x} \diamond A\right)\right)\left(\right.$ or $\left.\Pi x\left(R x \rightarrow\left(\mathcal{W}_{x} A \rightarrow \mathcal{B}_{x} M A\right)\right)\right)$, and (iii) $\Pi x\left(R x \rightarrow\left(\left(\mathcal{W}_{x} A \wedge \mathcal{B}_{x} \square(A \rightarrow B)\right) \rightarrow \mathcal{W}_{x} B\right)\right)$ (or $\Pi x\left(R x \rightarrow\left(\left(\mathcal{W}_{x} A \wedge \mathcal{B}_{x} U(A \rightarrow\right.\right.\right.$ $\left.\left.B)) \rightarrow \mathcal{W}_{x} B\right)\right)$ ). (iii) is one possible interpretation of the so-called hypothetical imperative that was introduced by $\mathrm{Kant}^{8}$. Hence, this is a philosophically very

\footnotetext{
${ }^{8}$ See Kant, 1785, p. 45; English translation in Paton, 1948, pp. 80-81. According to Kant, 'Who
} 
interesting principle. ${ }^{9}$ (i) and (ii) are also interesting. (i) is a theorem in every system that includes the tableau rules $T-d b 4$ (Table 18) and $T-U R$ (Table 15), or $T-d b 4, T-M \mathcal{B}$ (Table 17) and $T-F T R$ (Table 15); I will prove this in Section 5 ; (ii) is a theorem in every system that includes the tableau rules $T-M \mathcal{B}, T-\mathcal{W} P$ (Table 16) and $T-a b 4$ (Table 16); and (iii) is a theorem in every system that includes the tableau rules $T-M \mathcal{W}$ (Table 16) and $T-d T$ (Table 14); in Section 3.4, I will use a 'semantic' argument to show that (iii) is valid in every model that satisfies the semantic conditions $C-M \mathcal{W}$ (Table 4) and $C-d T$ (Table 3). Hence, it is not only possible to symbolise these principle in our systems, we can also investigate what we must assume to be able to prove that they are valid and what follows from them if we accept them. It appears to be impossible to do this in any other logics in the literature. In most systems, we cannot even symbolise such expressions as 'everyone who is such and such believes that...', 'someone who is such and such believes that...', 'everyone who is such and such wants it to be the case that...', 'someone who is such and such wants it to be the case that...', etc.

(i)-(iii) seem more or less intuitively plausible to me. Consider, for example, the following instance of (ii): if $x$ is perfectly rational, then $x$ wants to pass right through this stonewall only if $x$ believes that it is possible to pass right through this stonewall. This instance of (ii) can be symbolised in the following way in our systems: $\Pi x\left(R x \rightarrow\left(\mathcal{W}_{x} P x \rightarrow \mathcal{B}_{x} \diamond P x\right)\right)$, where ' $P x$ ' says that $x$ passes right through this stonewall. In Section 7, I will prove that this formula is a theorem in every system that includes $T-M \mathcal{B}, T-\mathcal{W} P$ and $T-a b 4$. If this sentence is not true, there will be someone, say $c$, such that $c$ wants to pass right through this stonewall even though it is not the case that $c$ believes that it is possible to pass right through this stonewall. It might even be the case that $c$ believes that it is impossible to pass right through this stonewall. Suppose that $c$ believes this and still wants to pass right through this stonewall. She starts walking and hits her head against the wall. Furthermore, suppose she does not change any beliefs as a consequence of this event. So, she keeps hitting her head against the wall, like a fly that repeatedly tries to pass through a window until it drops dead. It might be the case that $c$ wants to pass right through this stonewall because she wants to enter a garden and if it were possible to pass right through this stonewall this would be the quickest way to enter this garden. Suppose also that there is a gate just a few feet from the place

wills the end, wills (so far as reason has decisive influence on his actions) also the means which are indispensably necessary and in his power' and “'If I fully will the effect, I also will the action required for it" is analytic'.

${ }^{9}$ For more on the hypothetical imperative, see, for example, Bedke (2009), Broome (1999), Brunero (2010), Downie (1984), Feldman (1986, Chapter 5), Foot (1972), Gensler (1985), Greenspan (1975), Harsanyi (1958), Hill (1973, 1989), Korsgaard (2008), Marshall (1982), Shaver (2006), Schroeder (2004, 2005, 2009, 2015), Wallace (2001) and Way (2010).

Australasian Journal of Logic (16:3) 2019, Article no. 3 
where $c$ is and that it would be very easy to enter the garden through the gate. In this situation, it seems difficult to call $c$ perfectly rational. ${ }^{10}$

Reason 3. Our systems can be used to define many interesting concepts in a rigorous way, for example, the concepts of (perfect) self-infallibility, (perfect) self-acceptance and (perfect) self-awareness (see Section 5).

Reason 4. The systems in this paper can be used to explain the validity of several intuitively valid arguments that cannot be proved in any other system and they can be used to generate countermodels that show that an argument is not valid. Consider the following example of an intuitively valid (complex) argument:

Example 3. (The Doctoral Student Argument) Every student in the room wants to become a doctor some time in the future. Carl is a student in the room. So, Carl wants to become a doctor some time in the future. Carl believes that it is (historically) necessary that he will become a doctor some time in the future only if he studies hard. Hence, if Carl is perfectly rational (wise), Carl wants to study hard.

Many people think that arguments of this kind, which include a kind of meansend reasoning, are good. In some boulesic-doxastic systems introduced in this paper, the conclusion follows from the premises. Yet, we cannot find even a plausible symbolisation of this argument in any other system. In fact, the whole deduction includes two subarguments and two conclusions. In Section 7, I will show how we can symbolise these arguments and use semantic tableaux to prove that the first is valid in all systems in this paper and that the second is valid in every system that includes the tableau rules $T-d T$ and $T-M \mathcal{W}$ (see sections 4.2.7 and 4.2.9, Table 14 and Table 16).

Even though the argument in Example 3 (The Doctoral Student Argument) is intuitively plausible, the following argument appears to be invalid:

\footnotetext{
${ }^{10}$ It might be possible to think of bizarre scenarios where someone has (at least some) reasons to want something even though she believes that it is not possible. We can, for example, think of a situation where there is some mad scientist that is able to scan this person's brain and decide what she wants and believes. Suppose this scientist, as a part of some crazy experiment, says that he will punish this person if she does not want something she believes is impossible. Then this person might have some reasons to want something even though she does not believe that it is possible. But it is not obvious that those reasons are 'rational' reasons. We can perhaps say that this person in this case has 'pragmatic' or 'instrumental' or 'self-interested' reasons not to be perfectly rational. In a similar way someone can have 'external' reasons to believe in a contradiction. This does not necessarily entail that it is rational, reasonable or wise to believe in a contradiction, at least not in the sense that we are using these terms in the present paper. Whether or not this is a reasonable position, (ii) is a philosophically interesting principle that is worth exploring further. And without a boulesic-doxastic logic we cannot even symbolise principles of this kind in any plausible way. This is a strong reason to be interested in the systems in this paper.
} 
Example 4. (The Doctoral Student Argument II) Every student in the room wants to become a doctor some time in the future. Carl is a student in the room. Carl believes that it is (historically) necessary that he will become a doctor some time in the future only if he studies hard. Hence, Carl wants to study hard.

Note that in this argument the conclusion is not a conditional where the antecedent says that Carl is perfectly rational (wise). The conclusion is the categorical claim that Carl wants to study hard. But this proposition does not seem to follow from the premises. If Carl is not perfectly rational, he may want many things without wanting the things he believes are necessary means to the things he wants. From the first and the second premise, we can still conclude that Carl wants to become a doctor some time in the future. But from this sentence and the proposition that Carl believes that it is (historically) necessary that he will become a doctor some time in the future only if he studies hard, it does not follow that Carl wants to study hard. In Section 7, I will show how one can use semantic tableaux to generate countermodels and prove that this argument is not valid (in the class of all models).

Reason 5. Boulesic-doxastic logic can be used to solve several puzzles in a rather conservative and economical way. For example, we do not have to postulate any new entities, such as impossible worlds or impossible objects, to solve the problem of logical omniscience (see reason 1 above). The tableau rules for the propositional connectives, for the modal operators and for the (possibilist) quantifiers are standard. Furthermore, all normal doxastic systems assume that the domain (of agents) is non-empty. And since Belief of valid formulas holds in all those systems, they entail the existence of at least one individual that believes every valid sentence (at least if we assume that the domain only includes existing things). All systems in the present paper are compatible both with the existence and the nonexistence of a perfectly rational individual. Hence, they are ontologically more neutral than many systems in the literature. I therefore conclude that we have both very good technical and very good philosophical reasons to be interested in the systems that are developed in this paper.

The paper consists of seven main sections. Section 2 is about syntax and Section 3 about semantics. In Section 4, I introduce the proof theory of our systems, while Section 5 includes some examples of theorems. Section 6 contains soundness and completeness proofs for every non-augmented system, that is, every system without the propositional quantifiers. Finally, Section 7 includes some examples of valid and invalid arguments and principles. 


\section{Syntax}

\subsection{Alphabet}

Terms: variables $x_{1}, x_{2}, x_{3} \ldots$; constants (rigid designators) $k_{d_{1}}, k_{d_{2}}, k_{d_{3}}, \ldots$

Predicates: $n$-place predicate symbols $P_{n}^{1}, P_{n}^{2}, P_{n}^{3} \ldots$, for every natural number $n, E$ (existence), $R$ (rationality) and = (necessary identity).

Connectives: $\neg$ (negation), $\wedge$ (conjunction), $\vee($ disjunction $), \rightarrow$ (material implication) and $\leftrightarrow$ (material equivalence).

Operators: modal: $U$ (absolute necessity), $M$ (absolute possibility), $\square$ (historical necessity), $\diamond$ (historical possibility); boulesic: $\mathcal{W}$ (want), $\mathcal{A}$ (acceptability); doxastic: $\mathcal{B}$ (belief) and $\mathcal{C}$ (imaginability, conceivability).

Quantifiers: $\Pi$ (all) and $\Sigma$ (some).

Parentheses: ) and (.

We will use $x, y$ and $z \ldots$ for arbitrary variables, $a, b, c \ldots$ for arbitrary constants, $s$ and $t$ for arbitrary terms (with or without primes or subscripts) and $F_{n}, G_{n}$, $H_{n} \ldots$ for arbitrary $n$-place predicates (we will omit the subscript if it can be read off from the context).

\subsection{Languages}

The language $\mathcal{L}$ is the set of well-formed formulas generated by the following clauses:

(i) Any constant or variable is a term.

(ii) If $t_{1}, \ldots, t_{n}$ are any terms and $P$ is any $n$-place predicate, $P t_{1} \ldots t_{n}$ is an atomic formula.

(iii) If $t$ is a term, Et (' $t$ exists') is an atomic formula and $R t$ (' $t$ is perfectly rational') is an atomic formula.

(iv) If $s$ and $t$ are terms, then $s=t$ (' $s$ is identical with $t$ ') is an atomic formula.

(v) If $A$ and $B$ are formulas, so are $\neg A,(A \wedge B),(A \vee B),(A \rightarrow B)$ and $(A \leftrightarrow B)$.

(vi) If $A$ is a formula, so are $U A$ ('it is universally [or absolutely] necessary that $A^{\prime}$ ), $M A$ ('it is universally [or absolutely] possible that $A^{\prime}$ ), $\square A$ ('it is [historically] necessary that $A$ ') and $\diamond A$ ('it is [historically] possible that $A$ ').

(vii) If $D$ is any formula and $t$ is any term, then $\mathcal{W}_{t} D\left({ }^{\prime} t\right.$ wants it to be the case that $D$ ') and $\mathcal{A}_{t} D$ (' $t$ accepts that it is the case that $D$ ') are formulas.

(viii) If $D$ is any formula and $t$ is any term, then $\mathcal{B}_{t} D$ (' $t$ believes that $D$ ') and $\mathcal{C}_{t} D$ ('it is imaginable [or conceivable] to $t$ that $D$ ') are formulas.

(ix) If $A$ is any formula and $x$ is any variable, then $\Pi x A$ ('for every [possible] $\mathrm{x}: A^{\prime}$ ) and $\Sigma x A$ ('for some [possible] x: $A^{\prime}$ ) are formulas.

(x) Nothing else is a formula. 
$A, B, C, D \ldots$ represent arbitrary formulas, and $\Gamma, \Phi \ldots$ finite sets of closed formulas. The concepts of bound and free variables, and open and closed formulas, are defined as usual. $(A)[t / x]$ is the formula obtained by substituting $t$ for every free occurrence of $x$ in $A$. The definition is standard. Brackets are usually dropped if the result is not ambiguous.

The language $\mathcal{L}_{S u b}$ is the language $\mathcal{L}$ augmented with propositional quantifiers. To obtain $\mathcal{L}_{S u b}$, we augment our language with a set of propositional variables $X$, $Y, Z, W$ (with or without subscripts). Then we modify the formation-clauses, as standard. In particular, we add the following clause: if $A$ is any formula (in our extended language $\mathcal{L}_{S u b}$ ) and $X$ is any propositional variable, then $\Pi X A$ and $\Sigma X A$ are formulas. $(A)[B / X]$ is the result of uniformly replacing free occurrences of $X$ in $A$ by $B$ and $(A)\left[B_{1} / X_{1}, \ldots, B_{n} / X_{n}\right]$ is the result of simultaneously replacing free occurrences of $X_{1}$ in $A$ by $B_{1}, \ldots$, and $X_{n}$ in $A$ by $B_{n}$.

\section{Semantics}

\subsection{Models}

Definition 5. A model $\mathcal{M}$ is a structure $\langle D, W, \mathfrak{R}, \mathfrak{A}, \mathfrak{D}, v\rangle$, where $D$ is a non-empty set of individuals (the domain), $W$ is a non-empty set of possible worlds, $\mathfrak{R}$ is a binary alethic accessibility relation $(\mathfrak{R} \subseteq W \times W), \mathfrak{A}$ is a ternary boulesic accessibility relation $(\mathfrak{A} \subseteq D \times W \times W)$, $\mathfrak{D}$ is a ternary doxastic accessibility relation $(\mathfrak{D} \subseteq$ $D \times W \times W)$, and $v$ is an interpretation function.

Informally, $\mathfrak{R} \omega \omega^{\prime}$ says that the possible world $\omega^{\prime}$ is alethically (historically) accessible from the possible world $\omega, \mathfrak{A} \delta \omega \omega^{\prime}$ that the possible world $\omega^{\prime}$ is acceptable to the individual $\delta$ in (or relative to) the possible world $\omega$, or that $\delta$ accepts $\omega^{\prime}$ in (or relative to) $\omega$, and $\mathfrak{D} \delta \omega \omega^{\prime}$ that the possible world $\omega^{\prime}$ is doxastically accessible to the individual $\delta$ from the possible world $\omega$, or that $\delta$ can see $\omega^{\prime}$ from $\omega$.

The valuation function $v$ assigns every constant $c$ an element $v(c) \in D$, and every possible world $\omega \in W$ and $n$-place predicate $P$ a subset $v_{\omega}(P)$ (the extension of $P$ in $\omega$ ) of $D^{n}$. Thus, the constants are rigid designators while the extension of a predicate may change from world to world.

The extension of the identity predicate is the same in every possible world (in a model): $v_{\omega}(=)=\{\langle d, d\rangle: d \in D\}$. Hence, all identities (and non-identities) are both absolutely and historically necessary. The existence predicate $E$ functions as an ordinary predicate. ' $E c$ ' is true in a possible world iff $v(c)$ exists in this world.

The predicate $R$ has a special meaning. Informally, ' $R c$ ' says that $c$ is perfectly rational, perfectly reasonable or perfectly wise. Since $R$ functions as an 
ordinary predicate, an individual $\delta$ may be in $R$ 's extension in one possible world even though $\delta$ is not in $R$ 's extension in every possible world. Hence, the fact that an individual $\delta$ is perfectly rational, reasonable or wise in a possible world does not entail that $\delta$ is perfectly rational, reasonable or wise in every possible world. Exactly what it means to be perfectly rational, reasonable or wise will depend on the conditions we impose on the boulesic and doxastic accessibility relations (Section 3.3). $R$ plays an important role in the definitions of the truth conditions for our boulesic and doxastic operators.

The valuation function assigns extensions to so-called matrices. Given any closed boulesic or doxastic formula of the form $\mathcal{W}_{t} A, \mathcal{A}_{t} A, \mathcal{B}_{t} A$ or $\mathcal{C}_{t} A$, we shall construct its matrix as follows. Let $m$ be the least number greater than every $n$ such that $x_{n}$ occurs bound in $A$. From left to right, replace every occurrence of an individual constant with $x_{m}, x_{m+1}$, etc. The result is the formula's matrix. Consider the following examples: the matrix of $\mathcal{W}_{c} P d$ is $\mathcal{W}_{x_{1}} P x_{2}$; the matrix of $\mathcal{A}_{a} P c c$ is $\mathcal{A}_{x_{1}} P x_{2} x_{3}$; the matrix of $\mathcal{B}_{c}(F a \wedge G b c)$ is $\mathcal{B}_{x_{1}}\left(F x_{2} \wedge G x_{3} x_{4}\right)$; the matrix of $\mathcal{W}_{a} \Sigma x_{1}\left(F x_{1} \rightarrow G c\right)$ is $\mathcal{W}_{x_{2}} \Sigma x_{1}\left(F x_{1} \rightarrow G x_{3}\right)$; the matrix of $\mathcal{W}_{c} \mathcal{B}_{d} \Pi x_{2} P x_{2}$ is $\mathcal{W}_{x_{3}} \mathcal{B}_{x_{4}} \Pi x_{2} P x_{2}$, etc. $(A)\left[a_{1}, \ldots, a_{n} / x_{1}, \ldots, x_{n}\right]$ is the result of replacing every free occurrence of $x_{1}$ by $a_{1}, \ldots$, and every free occurrence of $x_{n}$ by $a_{n}$ in A. (A) $\left[a_{1}, \ldots, a_{n} / x_{1}, \ldots, x_{n}\right]$ will be abbreviated as $(A)\left[a_{1}, \ldots, a_{n} / \vec{x}\right]$. If $M$ is any matrix of the form $\mathcal{W}_{t} A, \mathcal{A}_{t} A, \mathcal{B}_{t} A$ or $\mathcal{C}_{t} A$ with free variables $x_{1}, \ldots, x_{n}$, then $v_{\omega}(M) \subseteq D^{n}$. Note that $M$ always includes at least one free variable. ${ }^{11}$ Let $M$ be a matrix where $x_{m}$ is the first free variable in $M$ and $a_{m}$ is the constant in $M\left[a_{1}, \ldots, a_{n} / \vec{x}\right]$ that replaces $x_{m}$. Then the truth conditions for closed boulesic or doxastic formulas of the form $M\left[a_{1}, \ldots, a_{n} / \vec{x}\right]$, when $v_{\omega}\left(R a_{m}\right)=0$, are defined in terms of the extension of $M$ in $\omega$ (see condition (ii) in Section 3.2 below). For some examples of matrices and how they function, see sections 3.2 and 7.

Let $\mathcal{M}$ be a model. Then the language of $\mathcal{M}, \mathcal{L}(\mathcal{M})$, is obtained by adding a constant $k_{d}$, such that $v\left(k_{d}\right)=d$, to the language for every member $d \in D$. Consequently, every object in the domain of a model has at least one name in our language.

\subsection{Truth conditions}

We now extend the valuation function. Every closed formula in $\mathcal{L}\left(\mathcal{L}_{S u b}\right)$ is assigned exactly one truth-value ( $1=$ True or $0=$ False), $v_{\omega}(A)$, in each world $\omega$ in a model $\mathcal{M}$. The truth conditions for the omitted truth-functional connectives are standard.

(i) $v_{\omega}\left(P a_{1} \ldots a_{n}\right)=1$ iff $\left\langle v\left(a_{1}\right), \ldots, v\left(a_{n}\right)\right\rangle \in v_{\omega}(P)$.

\footnotetext{
${ }^{11}$ The idea of using matrices is borrowed from Priest (2005, Ch. 1-2).
} 
Let $M$ be a matrix where $x_{m}$ is the first free variable in $M$ and $a_{m}$ is the constant in $M\left[a_{1}, \ldots, a_{n} / \vec{x}\right]$ that replaces $x_{m}$. Then the truth conditions for closed boulesic and doxastic formulas of the form $M\left[a_{1}, \ldots, a_{n} / \vec{x}\right]$, when $v_{\omega}\left(R a_{m}\right)=0$, are given in (ii) below.

(ii) $v_{\omega}\left(M\left[a_{1}, \ldots, a_{n} / \vec{x}\right]\right)=1$ iff $\left\langle v\left(a_{1}\right), \ldots, v\left(a_{n}\right)\right\rangle \in v_{\omega}(M)$.

(iii) $v_{\omega}(\neg A)=1$ iff $v_{\omega}(A)=0$.

(iv) $v_{\omega}(A \wedge B)=1$ iff $v_{\omega}(A)=1$ and $v_{\omega}(B)=1$.

(v) $v_{\omega}(U A)=1$ iff $\forall \omega^{\prime} \in W: v_{\omega^{\prime}}(A)=1$.

(vi) $v_{\omega}(M A)=1$ iff $\exists \omega^{\prime} \in W: v_{\omega^{\prime}}(A)=1$.

(vii) $v_{\omega}(\square A)=1$ iff $\forall \omega^{\prime} \in W$ s.t. $\mathfrak{R} \omega \omega^{\prime}: v_{\omega^{\prime}}(A)=1$.

(viii) $v_{\omega}(\diamond A)=1$ iff $\exists \omega^{\prime} \in W$ s.t. $\mathfrak{R} \omega \omega^{\prime}: v_{\omega^{\prime}}(A)=1$.

(ix) $v_{\omega}(\Pi x A)=1$ iff for all $k_{d} \in \mathcal{L}(\mathcal{M}), v_{\omega}\left(A\left[k_{d} / x\right]\right)=1$.

(x) $v_{\omega}(\Sigma x A)=1$ iff for some $k_{d} \in \mathcal{L}(\mathcal{M}), v_{\omega}\left(A\left[k_{d} / x\right]\right)=1$.

(xi) $v_{\omega}\left(\mathcal{B}_{a} D\right)=1$ iff for all $\omega^{\prime}$ such that $\mathfrak{D} v(a) \omega \omega^{\prime}: v_{\omega^{\prime}}(D)=1$, given that $v(a)$ is an element in $v_{\omega}(R)$, if $v(a)$ is not an element in $v_{\omega}(R)$, then $\mathcal{B}_{a} D$ is assigned a truth-value in $\omega$ in a way that does not depend on the value of $D$ (see condition (ii) above).

(xii) $v_{\omega}\left(\mathcal{C}_{a} D\right)=1$ iff for at least one $\omega^{\prime}$ such that $\mathfrak{D} v(a) \omega \omega^{\prime}: v_{\omega^{\prime}}(D)=1$, given that $v(a)$ is an element in $v_{\omega}(R)$, if $v(a)$ is not an element in $v_{\omega}(R)$, then $\mathcal{C}_{a} D$ is assigned a truth-value in $\omega$ in a way that does not depend on the value of $D$ (see condition (ii) above).

(xiii) $\mathcal{W}_{a} D$. The same as for $\mathcal{B}_{a} D$, except that we replace $\mathcal{B}$ by $\mathcal{W}$ and $\mathfrak{D}$ by $\mathfrak{A}$.

(xiv) $\mathcal{A}_{a} D$. The same as for $\mathcal{C}_{a} D$, except that we replace $\mathcal{C}$ by $\mathcal{A}$ and $\mathfrak{D}$ by $\mathfrak{A}$.

If $v(a)$ is not perfectly rational in a possible world, $\mathcal{W}_{a} D, \mathcal{A}_{a} D, \mathcal{B}_{a} D$ and $\mathcal{C}_{a} D$ behave as if they are predicates in this world; and if $v(a)$ is perfectly rational in a possible world, $\mathcal{W}_{a}, \mathcal{A}_{a}, \mathcal{B}_{a}$ and $\mathcal{C}_{a}$ behave as ordinary modal operators in this world. In other words, if $v(a)$ is not perfectly rational in a possible world, almost nothing of interest follows from the fact that $v(a)$ wants something, accepts something, believes something or finds something imaginable or conceivable, while all sorts of interesting facts follow if $v(a)$ is perfectly rational.

Let us return to The Triangle Scenario (see Example 1) that we mentioned in the introduction. This example will help explain conditions (ii) and (xi) above and the concept of a matrix. ${ }^{12}$ Recall that the propositions in The Triangle Scenario can be symbolised in the following way: $\mathcal{B}_{c} T t, \mathcal{B}_{c}(T t \rightarrow Q t), \neg \mathcal{B}_{c} Q t$ and $\mathcal{B}_{c} \neg Q t$, where ' $T$ ' stands for 'is a triangle', ' $Q$ ' stands for 'is equiangular', ' $c$ ' refers to Mike and ' $t$ ' refers to this figure. According to our systems, it is possible that there is a possible world in which all these sentences are true. We will now verify this.

\footnotetext{
${ }^{12}$ Since (xi) is (formally) similar to conditions (xii)-(xiv), this example will also help explain those conditions.
} 
$\mathcal{B}_{x_{1}} T x_{2}$ is the matrix of $\mathcal{B}_{c} T t, \mathcal{B}_{x_{1}}\left(T x_{2} \rightarrow Q x_{3}\right)$ is the matrix of $\mathcal{B}_{c}(T t \rightarrow Q t)$, $\mathcal{B}_{x_{1}} Q x_{2}$ is the matrix of $\mathcal{B}_{c} Q t$ and $\mathcal{B}_{x_{1}} \neg Q x_{2}$ is the matrix of $\mathcal{B}_{c} \neg Q t$. Now, consider the following model. $W=\left\{\omega_{0}\right\}, D=\{$ Mike, This Figure $\}$ and $\mathfrak{R}, \mathfrak{A}$ and $\mathfrak{D}$ are empty. $v(c)=$ Mike, $v(t)=$ This Figure, $v_{\omega_{0}}\left(\mathcal{B}_{x_{1}} T x_{2}\right)$ is the extension of $\mathcal{B}_{x_{1}} T x_{2}$ in $\omega_{0}, v_{\omega_{0}}\left(\mathcal{B}_{x_{1}}\left(T x_{2} \rightarrow Q x_{3}\right)\right)$ is the extension of $\mathcal{B}_{x_{1}}\left(T x_{2} \rightarrow Q x_{3}\right)$ in $\omega_{0}$, etc. Let $v_{\omega_{0}}\left(\mathcal{B}_{x_{1}} T x_{2}\right)$ include $\langle v(c), v(t)\rangle$, let $v_{\omega_{0}}\left(\mathcal{B}_{x_{1}}\left(T x_{2} \rightarrow Q x_{3}\right)\right)$ include $\langle v(c), v(t), v(t)\rangle$, let $v_{\omega_{0}}\left(\mathcal{B}_{x_{1}} Q x_{2}\right)$ be empty, and let $v_{\omega_{0}}\left(\mathcal{B}_{x_{1}} \neg Q x_{2}\right)$ include $\langle v(c), v(t)\rangle$. Let $R$ be empty in $\omega_{0}$, that is, assume that no-one is perfectly rational in $\omega_{0}$. Finally, let $T$ include $v(t)$ and let $Q$ be empty (for our purposes, it does not matter what is and what is not included in the extensions of $T$ and $Q$ ). In this model, $\mathcal{B}_{c} T t, \mathcal{B}_{c}(T t \rightarrow Q t), \neg \mathcal{B}_{c} Q t$ and $\mathcal{B}_{c} \neg Q t$ are all true in $\omega_{0}$. Let us verify that $\mathcal{B}_{c} T t$ and $\neg \mathcal{B}_{c} Q t$ are both true in $\omega_{0}$.

If $R c$ is false in $\omega_{0}$, then $\mathcal{B}_{x_{1}} T x_{2}\left[c, t / x_{1}, x_{2}\right]$ is true in $\omega_{0}$ iff $\langle v(c), v(t)\rangle$ is an element in $v_{\omega_{0}}\left(\mathcal{B}_{x_{1}} T x_{2}\right)$ (see condition (ii) above). Since $R c$ is false in $\omega_{0}$, it follows that $\mathcal{B}_{x_{1}} T x_{2}\left[c, t / x_{1}, x_{2}\right]$ is true in $\omega_{0}$ iff $\langle v(c), v(t)\rangle$ is in $v_{\omega_{0}}\left(\mathcal{B}_{x_{1}} T x_{2}\right) .\langle v(c), v(t)\rangle$ is in $v_{\omega_{0}}\left(\mathcal{B}_{x_{1}} T x_{2}\right)$. Hence, $\mathcal{B}_{x_{1}} T x_{2}\left[c, t / x_{1}, x_{2}\right]$ is true in $\omega_{0}$. $\mathcal{B}_{x_{1}} T x_{2}\left[c, t / x_{1}, x_{2}\right]=$ $\mathcal{B}_{c} T t$. Consequently, $\mathcal{B}_{c} T t$ is true in $\omega_{0}$.

If $R c$ is false in $\omega_{0}$, then $\mathcal{B}_{x_{1}} Q x_{2}\left[c, t / x_{1}, x_{2}\right]$ is true in $\omega_{0}$ iff $\langle v(c), v(t)\rangle$ is an element in $v_{\omega_{0}}\left(\mathcal{B}_{x_{1}} Q x_{2}\right)$. Since $R c$ is false in $\omega_{0}$, it follows that $\mathcal{B}_{x_{1}} Q x_{2}\left[c, t / x_{1}, x_{2}\right]$ is true in $\omega_{0}$ iff $\langle v(c), v(t)\rangle$ is an element in $v_{\omega_{0}}\left(\mathcal{B}_{x_{1}} Q x_{2}\right) .\langle v(c), v(t)\rangle$ is not an element in $v_{\omega_{0}}\left(\mathcal{B}_{x_{1}} Q x_{2}\right)$. Hence, $\mathcal{B}_{x_{1}} Q x_{2}\left[c, t / x_{1}, x_{2}\right]$ is not true in $\omega_{0}$, i.e. it is false in $\omega_{0}$. Therefore, $\neg \mathcal{B}_{x_{1}} Q x_{2}\left[c, t / x_{1}, x_{2}\right]$ is true in $\omega_{0} . \neg \mathcal{B}_{x_{1}} Q x_{2}\left[c, t / x_{1}, x_{2}\right]=\neg \mathcal{B}_{c} Q t$. It follows that $\neg \mathcal{B}_{c} Q t$ is true in $\omega_{0}$.

In most doxastic systems in the literature we cannot find any plausible symbolisation of this scenario. In those systems, the set that includes $\mathcal{B}_{c} T t, \mathcal{B}_{c}(T t \rightarrow Q t)$ and $\neg \mathcal{B}_{c} Q t$ is inconsistent. If Mike begins to think about his beliefs, he might become aware of the fact that they are inconsistent. Hence, he might come to reject some of his beliefs. He might, for example, reject the false belief that all triangles are equiangular. An equiangular triangle is a triangle where all three interior angles are equal in measure and it is not the case that all three interior angles are equal in measure in all triangles. This does not entail that his beliefs were consistent all along. This example illustrates the fact that not every instance of the following problematic formula is a theorem in our systems: $\Pi x\left(\left(\mathcal{B}_{x} A \wedge \mathcal{B}_{x}(A \rightarrow B)\right) \rightarrow \mathcal{B}_{x} B\right)$ (see the introduction).

The propositional quantifiers $\Pi$ and $\Sigma$ are a kind of 'substitutional' quantifiers. Intuitively, $\Pi X A$ is true iff every substitution instance of $A$ is true, and $\Sigma X A$ is true iff some substitution instance of $A$ is true. Substitutions are subject to the usual provisos; no free variable should be bound by any quantifier, etc. To avoid circularity, we shall forbid substitutions that include the propositional quantifiers. (Alternatively, we can construct a hierarchy of propositional quantifiers in which the 
substitution instances for a propositional quantifier at a given level contain propositional quantifiers only from lower levels.) In tableau systems that include the tableau rules for the propositional quantifiers, we will allow that open formulas occur in a tree. However, we will only consider substitutions where the free variables are propositional variables. Open sentences are neither true nor false. So, we will define the truth conditions for various formulas in $\mathcal{L}_{S u b}$ with respect to an assignment, where an assignment, $\alpha$, is a function which assigns to each propositional variable a sentence in $\mathcal{L}$. The assignment $\alpha^{\prime}$ is an $X$-variant of the assignment $\alpha$ if $\alpha$ and $\alpha^{\prime}$ agree on all variables except possibly the variable $X$. If $A$ is a formula whose free (propositional) variables are $X_{1}, \ldots, X_{n}$, then $A$ is true in the possible world $\omega$ in the model $\mathcal{M}$ with respect to the assignment $\alpha$ just in case (A) $\left[\alpha\left(X_{1}\right) / X_{1}, \ldots, \alpha\left(X_{n}\right) / X_{n}\right]$. ПXA is true in $\omega$ in $\mathcal{M}$ with respect to $\alpha$ just in case $A$ is true in $\omega$ in $\mathcal{M}$ for every assignment $\alpha^{\prime}$ that is an $X$-variant of $\alpha$. The truth conditions for $\Sigma X A$ are similar. The truth conditions for the constructs that already appear in $\mathcal{L}$ are the same. Since sentences do not contain any free variables, we can continue to talk about sentences as true and false in a possible world in a model (without mentioning any assignments).

The concepts of semantic validity, entailment, satisfiability and so on can be defined in the usual way (see the introduction for some relevant references).

\subsection{Conditions on models}

In this section, I will introduce some conditions that might be imposed on our models. These conditions correspond to some tableau rules in Section 4.2 and to various interesting theorems discussed in Section $5 .{ }^{13}$

\subsubsection{Conditions on the relation $\mathfrak{R}$}

\begin{tabular}{l|l}
\hline Condition & Formalisation of condition \\
\hline$C-a T$ & $\forall x \mathfrak{R} x x$ \\
$C-a D$ & $\forall x \exists y \mathfrak{R} x y$ \\
$C-a B$ & $\forall x \forall y(\mathfrak{R} x y \rightarrow \mathfrak{R} y x)$ \\
$C-a 4$ & $\forall x \forall y \forall z((\mathfrak{R} x y \wedge \mathfrak{R} y z) \rightarrow \mathfrak{R} x z)$ \\
$C-a 5$ & $\forall x \forall y \forall z((\mathfrak{R} x y \wedge \mathfrak{R} x z) \rightarrow \mathfrak{R} y z)$ \\
\hline \multicolumn{2}{c}{ Table 1 }
\end{tabular}

\footnotetext{
${ }^{13}$ Which conditions should we accept? This seems to be something of an open question. I would be willing to defend most of the conditions, but some combinations might generate systems that are intuitively too strong (see Section 4.3). We might want to use different combinations of conditions for different purposes. Similar remarks apply to the tableau rules in Section 4.2 and to the theorems in Section 5.
} 


\subsubsection{Conditions on the relation $\mathfrak{A}$}

\begin{tabular}{l|l}
\hline Condition & Formalisation of condition \\
\hline$C-b D$ & $\forall d \forall x \exists y \mathfrak{A} d x y$ \\
$C-b 4$ & $\forall d \forall x \forall y \forall z((\mathfrak{A} d x y \wedge \mathfrak{A} d y z) \rightarrow \mathfrak{A} d x z)$ \\
$C-b 5$ & $\forall d \forall x \forall y \forall z((\mathfrak{A} d x y \wedge \mathfrak{A} d x z) \rightarrow \mathfrak{A} d y z)$ \\
$C-b T^{\prime}$ & $\forall d \forall x \forall y(\mathfrak{A} d x y \rightarrow \mathfrak{A} d y y)$ \\
$C-b B^{\prime}$ & $\forall d \forall x \forall y \forall z((\mathfrak{A} d x y \wedge \mathfrak{A} d y z) \rightarrow \mathfrak{A} d z y)$ \\
\hline \multicolumn{2}{c}{ Table 2}
\end{tabular}

\subsubsection{Conditions on the relation $\mathfrak{D}$}

\begin{tabular}{l|l}
\hline Condition & Formalisation of condition \\
\hline$C-d O$ & $\forall d \forall x \forall y(\mathfrak{D} d x y \rightarrow x=y)$ \\
$C-d T$ & $\forall d \forall x \mathfrak{D} d x x$ \\
$C-d D$ & $\forall d \forall x \exists y \mathfrak{D} d x y$ \\
$C-d B$ & $\forall d \forall x \forall y(\mathfrak{D} d x y \rightarrow \mathfrak{D} d y x)$ \\
$C-d 4$ & $\forall d \forall x \forall y \forall z((\mathfrak{D} d x y \wedge \mathfrak{D} d y z) \rightarrow \mathfrak{D} d x z)$ \\
$C-d 5$ & $\forall d \forall x \forall y \forall z((\mathfrak{D} d x y \wedge \mathfrak{D} d x z) \rightarrow \mathfrak{D} d y z)$ \\
$C-d T^{\prime}$ & $\forall d \forall x \forall y(\mathfrak{D} d x y \rightarrow \mathfrak{D} d y y)$ \\
$C-d B^{\prime}$ & $\forall d \forall x \forall y \forall z((\mathfrak{D} d x y \wedge \mathfrak{D} d y z) \rightarrow \mathfrak{D} d z y)$ \\
\hline \multicolumn{2}{c}{ Table 3}
\end{tabular}

\subsubsection{Conditions concerning the relation between $\mathfrak{R}$ and $\mathfrak{A}$}

\begin{tabular}{l|l}
\hline Condition & Formalisation of condition \\
\hline$C-M \mathcal{W}$ & $\forall d \forall x \forall y(\mathfrak{A} d x y \rightarrow \mathfrak{R} x y)$ \\
$C-\mathcal{W P}$ & $\forall d \forall x \exists y(\mathfrak{A} d x y \wedge \mathfrak{R} x y)$ \\
$C-M \mathcal{W}^{\prime}$ & $\forall d \forall x \forall y \forall z((\mathfrak{A} d x y \wedge \mathfrak{A} d y z) \rightarrow \mathfrak{R} y z)$ \\
$C-\mathcal{W} P^{\prime}$ & $\forall d \forall x \forall y(\mathfrak{A} d x y \rightarrow \exists z(\mathfrak{A} d y z \wedge \mathfrak{R} y z))$ \\
$C-a b 4$ & $\forall d \forall x \forall y \forall z((\mathfrak{R} x y \wedge \mathfrak{A} d y z) \rightarrow \mathfrak{A} d x z)$ \\
$C-a b 5$ & $\forall d \forall x \forall y \forall z((\mathfrak{R} x y \wedge \mathfrak{A} d x z) \rightarrow \mathfrak{A} d y z)$ \\
$C-\mathcal{A} M P$ & $\forall d \forall x \forall y \forall z((\mathfrak{A} d x y \wedge \mathfrak{R} x z) \rightarrow \exists w(\mathfrak{R} y w \wedge \mathfrak{A} d z w))$ \\
$C-\mathcal{W} M P$ & $\forall d \forall x \forall y \forall z((\mathfrak{R} x y \wedge \mathfrak{A} d y z) \rightarrow \exists w(\mathfrak{A} d x w \wedge \mathfrak{R} w z))$ \\
$C-M \mathcal{W} P$ & $\forall d \forall x \forall y \forall z((\mathfrak{A} d x y \wedge \mathfrak{R} y z) \rightarrow \exists w(\mathfrak{R} x w \wedge \mathfrak{A} d w z))$ \\
\hline \multicolumn{2}{|c}{ Table 4}
\end{tabular}




\subsubsection{Conditions concerning the relation between $\mathfrak{R}$ and $\mathfrak{D}$}

\begin{tabular}{l|l}
\hline Condition & Formalisation of condition \\
\hline$C-M \mathcal{B}$ & $\forall d \forall x \forall y(\mathfrak{D} d x y \rightarrow \mathfrak{R} x y)$ \\
$C-\mathcal{B P}$ & $\forall d \forall x \exists y(\mathfrak{D} d x y \wedge \mathfrak{R} x y)$ \\
$C-M \mathcal{B}^{\prime}$ & $\forall d \forall x \forall y \forall z((\mathfrak{D} d x y \wedge \mathfrak{D} d y z) \rightarrow \mathfrak{R} y z)$ \\
$C-\mathcal{B} P^{\prime}$ & $\forall d \forall x \forall y(\mathfrak{D} d x y \rightarrow \exists z(\mathfrak{D} d y z \wedge \mathfrak{R} y z))$ \\
$C-\mathcal{B} M$ & $\forall d \forall x \forall y(\mathfrak{R} x y \rightarrow \mathfrak{D} d x y)$ \\
$C-a d 4$ & $\forall d \forall x \forall y \forall z((\mathfrak{R} x y \wedge \mathfrak{D} d y z) \rightarrow \mathfrak{D} d x z)$ \\
$C-a d 5$ & $\forall d \forall x \forall y \forall z((\mathfrak{R} x y \wedge \mathfrak{D} d x z) \rightarrow \mathfrak{D} d y z)$ \\
$C-\mathcal{C} M P$ & $\forall d \forall x \forall y \forall z((\mathfrak{D} d x y \wedge \mathfrak{R} x z) \rightarrow \exists w(\mathfrak{R} y w \wedge \mathfrak{D} d z w))$ \\
$C-\mathcal{B} M P$ & $\forall d \forall x \forall y \forall z((\mathfrak{R} x y \wedge \mathfrak{D} d y z) \rightarrow \exists w(\mathfrak{D} d x w \wedge \mathfrak{R} w z))$ \\
$C-M \mathcal{B} P$ & $\forall d \forall x \forall y \forall z((\mathfrak{D} d x y \wedge \mathfrak{R} y z) \rightarrow \exists w(\mathfrak{R} x w \wedge \mathfrak{D} d w z))$ \\
\hline \multicolumn{2}{|c}{ Table 5}
\end{tabular}

\subsubsection{Conditions concerning the relation between $\mathfrak{A}$ and $\mathfrak{D}$}

\begin{tabular}{l|l}
\hline Condition & Formalisation of condition \\
\hline$C-\mathcal{W B}$ & $\forall d \forall x \forall y(\mathfrak{D} d x y \rightarrow \mathfrak{A} d x y)$ \\
$C-\mathcal{B W}$ & $\forall d \forall x \forall y(\mathfrak{A} d x y \rightarrow \mathfrak{D} d x y)$ \\
$C-\mathcal{W C}$ & $\forall d \forall x \exists y(\mathfrak{A} d x y \wedge \mathfrak{D} d x y)$ \\
$C-\mathcal{B A}$ & $\forall d \forall x \exists y(\mathfrak{D} d x y \wedge \mathfrak{A} d x y)$ \\
$C-\mathcal{B W} \mathcal{B}$ & $\forall d \forall x \forall y(\mathfrak{D} d x y \rightarrow \mathfrak{A} d y y)$ \\
$C-\mathcal{W B} \mathcal{W}$ & $\forall d \forall x \forall y(\mathfrak{A} d x y \rightarrow \mathfrak{D} d y y)$ \\
$C-d b 4$ & $\forall d \forall x \forall y \forall z((\mathfrak{D} d x y \wedge \mathfrak{A} d y z) \rightarrow \mathfrak{A} d x z)$ \\
$C-d b 5$ & $\forall d \forall x \forall y \forall z((\mathfrak{D} d x y \wedge \mathfrak{A} d x z) \rightarrow \mathfrak{A} d y z)$ \\
$C-b d 4$ & $\forall d \forall x \forall y \forall z((\mathfrak{A} d x y \wedge \mathfrak{D} d y z) \rightarrow \mathfrak{D} d x z)$ \\
$C-b d 5$ & $\forall d \forall x \forall y \forall z((\mathfrak{A} d x y \wedge \mathfrak{D} d x z) \rightarrow \mathfrak{D} d y z)$ \\
$C-\mathcal{W W} \mathcal{B}$ & $\forall d \forall x \forall y \forall z((\mathfrak{A} d x y \wedge \mathfrak{D} d y z) \rightarrow \mathfrak{A} d x z)$ \\
$C-\mathcal{B B} \mathcal{W}$ & $\forall d \forall x \forall y \forall z((\mathfrak{D} d x y \wedge \mathfrak{A} d y z) \rightarrow \mathfrak{D} d x z)$ \\
$C-\mathcal{A B P}$ & $\forall d \forall x \forall y \forall z((\mathfrak{D} d x y \wedge \mathfrak{A} d x z) \rightarrow \exists w(\mathfrak{A} d y w \wedge \mathfrak{D} d z w))$ \\
$C-\mathcal{C W} P$ & $\forall d \forall x \forall y \forall z((\mathfrak{A} d x y \wedge \mathfrak{D} d x z) \rightarrow \exists w(\mathfrak{D} d y w \wedge \mathfrak{A} d z w))$ \\
$C-\mathcal{B W} P$ & $\forall d \forall x \forall y \forall z((\mathfrak{A} d x y \wedge \mathfrak{D} d y z) \rightarrow \exists w(\mathfrak{D} d x w \wedge \mathfrak{A} d w z))$ \\
$C-\mathcal{W} \mathcal{B} P$ & $\forall d \forall x \forall y \forall z((\mathfrak{D} d x y \wedge \mathfrak{A} d y z) \rightarrow \exists w(\mathfrak{A} d x w \wedge \mathfrak{D} d w z))$ \\
$C-\mathcal{W} O$ & $\forall d \forall x \forall y \forall z((\mathfrak{A} d x y \wedge \mathfrak{D} d y z) \rightarrow y=z)$ \\
\hline \multicolumn{2}{|c}{ Table 6}
\end{tabular}

$C-\mathcal{W C}$ and $C-\mathcal{B A}$ are logically equivalent. I mention both conditions since I will associate them with different tableau rules and theorems (see sections 4.2 and 5). The same goes for $C-\mathcal{A B P}$ and $C-\mathcal{C W} P$. 


\subsubsection{Conditions concerning the relation between $\mathfrak{R}, \mathfrak{A}$ and $\mathfrak{D}$}

\begin{tabular}{l|l}
\hline Condition & Formalisation of condition \\
\hline$C-W N I$ & $\forall d \forall x \forall y \forall z((\mathfrak{A} d x y \wedge \mathfrak{R} y z) \rightarrow \mathfrak{D} d y z)$ \\
$C-W N O$ & $\forall d \forall x \forall y \forall z((\mathfrak{A} d x y \wedge \mathfrak{D} d y z) \rightarrow \mathfrak{R} y z)$ \\
$C-W B P$ & $\forall d \forall x \forall y \forall z(\mathfrak{A} d x y \rightarrow \exists z(\mathfrak{D} d y z \wedge \mathfrak{R} y z))$ \\
$C-B W P$ & $\forall d \forall x \forall y \forall z(\mathfrak{D} d x y \rightarrow \exists z(\mathfrak{A} d y z \wedge \mathfrak{R} y z))$ \\
$C-B M W$ & $\forall d \forall x \forall y \forall z((\mathfrak{D} d x y \wedge \mathfrak{A} d y z) \rightarrow \mathfrak{R} y z)$ \\
\hline \multicolumn{2}{c}{ Table 7 }
\end{tabular}

\subsubsection{Conditions on the valuation function $v$ in a model}

\begin{tabular}{l|l}
\hline Condition & Formalisation of condition \\
\hline$C-F T R$ & $\begin{array}{l}\text { If } \Re \omega_{1} \omega_{2} \text { and } R c \text { is true in } \omega_{1}, \\
\left.\text { then } R c \text { is true in } \omega_{2} \text { (for any } c\right) .\end{array}$ \\
$C-U R$ & If $R c$ is true in $\omega_{1}$, then $R c$ is true in $\omega_{2}$ (for any $c$ ). \\
\hline
\end{tabular}

\subsection{An example of a valid formula}

In this section, I will show that the formalisation of sentence (iii) in Example 2 in the introduction is valid in every model that satisfies the semantic conditions $C-M \mathcal{W}$ (Table 4) and $C-d T$ (Table 3). Recall that (iii) is the following principle: If $x$ is perfectly rational (wise), then if $x$ wants it to be the case that $A$ and $x$ believes that it is necessary that if $A$ then $B$ then $x$ wants it to be the case that $B$. This principle can be symbolised in the following way in our formal language: $\Pi x\left(R x \rightarrow\left(\left(\mathcal{W}_{x} A \wedge \mathcal{B}_{x} \square(A \rightarrow B)\right) \rightarrow \mathcal{W}_{x} B\right)\right)$. To establish this, assume that this formula is not true in some possible world $\omega$ in some model $\mathcal{M}$ that satisfies $C-M \mathcal{W}$ and $C-d T$. Then $R c, \mathcal{W}_{c} A$ and $\left.\mathcal{B}_{x} \square(A \rightarrow B)\right)$ are true in $\omega$ in $\mathcal{M}$, while $\mathcal{W}_{c} B$ is false in $\omega$ in $\mathcal{M}$. Since $\mathcal{W}_{c} B$ is false in $\omega$ in $\mathcal{M}$ and $c$ is perfectly rational in $\omega$ in $\mathcal{M}$, there is a possible world $\omega^{\prime}$ in $\mathcal{M}$ that is boulesically accessible to $c$ from $\omega$ in which $B$ is false. Therefore, $A$ is true in $\omega^{\prime}$ in $\mathcal{M}$. For $c$ is perfectly rational in $\omega$ in $\mathcal{M}, \omega^{\prime}$ is boulesically accessible to $c$ from $\omega$ and $\mathcal{W}_{c} A$ is true in $\omega$ in $\mathcal{M}$. Hence, $\omega^{\prime}$ is alethically accessible from $\omega$, for $\mathcal{M}$ satisfies $C-M \mathcal{W}$. Furthermore, $\omega$ is doxastically accessible to $c$ from itself since $\mathcal{M}$ satisfies $T-d T$. Since $\omega$ is doxastically accessible to $c$ from itself, $c$ is perfectly rational in $\omega$ and $\left.\mathcal{B}_{x} \square(A \rightarrow B)\right)$ is true in $\omega$ in $\mathcal{M}, \square(A \rightarrow B)$ is true in $\omega$ in $\mathcal{M}$. It follows that $A \rightarrow B$ is true in $\omega^{\prime}$ in $\mathcal{M}$, for $\omega^{\prime}$ is alethically accessible from $\omega$. Hence, $B$ is true in $\omega^{\prime}$ in $\mathcal{M}$ (by propositional logic). But this is absurd. Consequently, our assumption cannot be true. In other words, $\Pi x\left(R x \rightarrow\left(\left(\mathcal{W}_{x} A \wedge \mathcal{B}_{x} \square(A \rightarrow B)\right) \rightarrow \mathcal{W}_{x} B\right)\right)$ is valid 
in $\mathcal{M}$. Since, $\omega$ and $\mathcal{M}$ were arbitrary, it follows that $\Pi x\left(R x \rightarrow\left(\left(\mathcal{W}_{x} A \wedge \mathcal{B}_{x} \square(A \rightarrow\right.\right.\right.$ $\left.\left.B)) \rightarrow \mathcal{W}_{x} B\right)\right)$ is valid in every model that satisfies $C-M \mathcal{W}$ and $C-d T$. Q.E.D.

\subsection{Model classes and the logic of a class of models}

Let $\mathcal{M}\left(C_{1}, \ldots, C_{n}\right)$ be the class of all models that satisfy the conditions $C_{1}, \ldots, C_{n}$. For example, $\mathcal{M}(C-d D, C-d b 4, C-b d 5)$ is the class of all models that satisfy the conditions $C-d D, C-d b 4$ and $C-b d 5$.

By imposing different conditions on our models we can define a set of logical systems. The set of all sentences in $\mathcal{L}$ that are valid in a class of models $\mathcal{M}$ is called the (logical) system (or logic) of $\mathcal{M}$, and in symbols, $\mathcal{S}(\mathcal{M})$. For example, $\mathcal{S}(\mathcal{M}(C-d D, C-d b 4, C-b d 5))$ (the system of $\mathcal{M}(C-d D, C-d b 4, C-b d 5))$ is the class of sentences in $\mathcal{L}$ that are valid in the class of all models that satisfy the conditions $C-d D, C-d b 4$ and $C-b d 5$. In an 'augmented system', we use $\mathcal{L}_{S u b}$ instead.

\section{Proof theory}

\subsection{Semantic tableaux}

The concepts of semantic tableau, branch, open and closed branch, proof, derivation, etc. are defined in a standard way. For more on the tableau method, see the references in the introduction.

\subsection{Tableau rules}

\subsubsection{Propositional rules}

I will use the same propositional rules as those in Priest (2008). Let us call them $(\wedge),(\neg \wedge)$, and so on. 


\subsubsection{Basic alethic rules (ba-rules)}

\begin{tabular}{c|c|c|c}
\hline$U$ & $M$ & $\square$ & $\diamond$ \\
\hline$U A, i$ & $M A, i$ & $\square A, i$ & $\diamond A, i$ \\
$\downarrow$ & $\downarrow$ & $i r j$ & $\downarrow$ \\
$A, j$ & $A, j$ & $\downarrow$ & $i r j$ \\
for any $j$ & where $j$ is new & $A, j$ & $A, j$ \\
& & & where $j$ is new \\
\hline$\neg U$ & $\neg M$ & $\neg \square$ & $\neg \diamond$ \\
\hline$\neg U A, i$ & $\neg M A, i$ & $\neg \square A, i$ & $\neg \diamond A, i$ \\
$\downarrow$ & $\downarrow$ & $\downarrow$ & $\downarrow$ \\
$M \neg A, i$ & $U \neg A, i$ & $\diamond \neg A, i$ & $\square \neg A, i$ \\
\hline
\end{tabular}

Table 9

\subsubsection{Basic boulesic and doxastic rules (bb-rules and bd-rules)}

\begin{tabular}{c|c|c|c}
\hline $\mathcal{W}$ & $\mathcal{A}$ & $\mathcal{B}$ & $\mathcal{C}$ \\
\hline$R c, i$ & $R c, i$ & $R c, i$ & $R c, i$ \\
$\mathcal{W}_{c} B, i$ & $\mathcal{A}_{c} B, i$ & $\mathcal{B}_{c} B, i$ & $\mathcal{C}_{c} B, i$ \\
$i A c j$ & $\downarrow$ & $i D c j$ & $\downarrow$ \\
$\downarrow$ & $i A c j$ & $\downarrow$ & $i D c j$ \\
$B, j$ & $B, j$ & $B, j$ & $B, j$ \\
& where $j$ is new & & where $j$ is new \\
\hline$\neg \mathcal{W}$ & $\neg \mathcal{A}$ & $\neg \mathcal{B}$ & $\neg \mathcal{C}$ \\
\hline$R c, i$ & $R c, i$ & $R c, i$ & $R c, i$ \\
$\neg \mathcal{W}_{c} B, i$ & $\neg \mathcal{A}_{c} B, i$ & $\neg \mathcal{B}_{c} B, i$ & $\neg \mathcal{C}_{c} B, i$ \\
$\downarrow$ & $\downarrow$ & $\downarrow$ & $\downarrow$ \\
$\mathcal{A}_{c} \neg B, i$ & $\mathcal{W}_{c} \neg B, i$ & $\mathcal{C}_{c} \neg B, i$ & $\mathcal{B}_{c} \neg B, i$ \\
\hline \multicolumn{4}{|r}{} \\
\hline \multicolumn{2}{|c}{}
\end{tabular}

Table 10

Intuitively, ' $R c, i$ ' in the rules in this section says that the individual denoted by ' $c$ ' is perfectly rational in the possible world denoted by ' $i$ ', ' $i A c j$ ' says that the possible world denoted by ' $j$ ' is acceptable to ' $c$ ' in ' $i$ ', and ' $i D c j$ ' says that ' $j$ ' is conceivable or imaginable to ' $c$ ' in ' $i$ '. All rules of this kind in this section hold for every constant $c$, that is, ' $c$ ' can be replaced by any constant in these rules. 


\subsubsection{Possibilist quantifiers}

\begin{tabular}{c|c|c|c}
\hline$\Pi$ & $\Sigma$ & $\neg \Pi$ & $\neg \Sigma$ \\
\hline$\Pi x A, i$ & $\Sigma x A, i$ & $\neg \prod x A, i$ & $\neg \Sigma x A, i$ \\
$\downarrow$ & $\downarrow$ & $\downarrow$ & $\downarrow$ \\
$A[a / x], i$ & $A[c / x], i$ & $\Sigma x \neg A, i$ & $\Pi x \neg A, i$ \\
for every constant $a$ \\
$\begin{array}{c}\text { on the branch, } \\
\text { where } c \text { is new } \\
\text { to the branch }\end{array}$ & & \\
$\begin{array}{c}\text { a new if there are no } \\
\text { constants on the branch }\end{array}$ & & & \\
\hline
\end{tabular}

Table 11

The terms $a$ and $c$ in the quantifier rules are rigid constants-we never instantiate with variables; $a$ is any constant on the branch and $c$ is a constant new to the branch.

\subsubsection{Alethic accessibility rules (a-rules)}

\begin{tabular}{c|c|c|c|c}
\hline$T-a D$ & $T-a T$ & $T-a B$ & $T-a 4$ & $T-a 5$ \\
\hline$i$ & $i$ & $i r j$ & $i r j$ & $i r j$ \\
$\downarrow$ & $\downarrow$ & $\downarrow$ & $j r k$ & $i r k$ \\
$i r j$ & $i r i$ & $j r i$ & $\downarrow$ & $\downarrow$ \\
where $j$ is new & & & $i r k$ & $j r k$ \\
\hline
\end{tabular}

Table 12

\subsubsection{Boulesic accessibility rules (b-rules)}

\begin{tabular}{c|c|c|c|c}
\hline$T-b D$ & $T-b 4$ & $T-b 5$ & $T-b T^{\prime}$ & $T-b B^{\prime}$ \\
\hline$i$ & $i A c j$ & $i A c j$ & $i A c j$ & $i A c j$ \\
$\downarrow$ & $j A c k$ & $i A c k$ & $\downarrow$ & $j A c k$ \\
$i A c j$ & $\downarrow$ & $\downarrow$ & $j A c j$ & $\downarrow$ \\
where $j$ is new & $i A c k$ & $j A c k$ & & $k A c j$ \\
\hline
\end{tabular}

Table 13 
4.2.7 Doxastic accessibility rules (d-rules)

\begin{tabular}{c|c|c|c|c|c|c|c}
\hline$T-d O$ & $T-d T$ & $T-d B$ & $T-d D$ & $T-d 4$ & $T-d 5$ & $T-d T^{\prime}$ & $T-d B^{\prime}$ \\
\hline$i D c j$ & $i$ & $i D c j$ & $i$ & $i D c j$ & $i D c j$ & $i D c j$ & $i D c j$ \\
$\downarrow$ & $\downarrow$ & $\downarrow$ & $\downarrow$ & $j D c k$ & $i D c k$ & $\downarrow$ & $j D c k$ \\
$i=j$ & $i D c i$ & $j D c i$ & $i D c j$ & $\downarrow$ & $\downarrow$ & $j D c j$ & $\downarrow$ \\
& & & where $j$ & $i D c k$ & $j D c k$ & & $k D c j$ \\
& & & is new & & & & \\
\hline
\end{tabular}

Table 14

\subsubsection{Transfer-rules, and the CUT-rule (CUT)}

\begin{tabular}{c|c|c}
\hline$T-F T R$ & $T-U R$ & $C U T$ \\
\hline$R c, i$ & $R c, i$ & $i$ \\
$i r j$ & $\downarrow$ & $\swarrow \searrow$ \\
$\downarrow$ & $R c, j$ & $A, i \neg A, i$ \\
$R c, j$ & for any $j$ & for every $A$ and $i$ \\
\hline \multicolumn{3}{|c}{ Table 15 }
\end{tabular}

It is possible to replace the $C U T$ rule by a weaker rule, $C U T R$. In $C U T R$ ' $A$ ' (in $C U T$ ) is replaced by ' $R c$ ' where $c$ is a constant (that occurs as an index to some boulesic operator) on the branch. In fact, in the completeness proofs (see Section 6) we do not need $C U T$ if our systems include $C U T R$. In Section 7, we will also assume that our weakest system $\mathcal{B D}$ (see Section 4.3) includes $C U T R$ and not $C U T$. However, $C U T$ is often more useful in proving theorems and deriving non-primitive rules. 
4.2.9 Alethic-boulesic accessibility rules (ab-rules)

\begin{tabular}{|c|c|c|c|c|}
\hline$T-M \mathcal{W}$ & $T-M \mathcal{W}^{\prime}$ & $T-\mathcal{W} P$ & $T-\mathcal{W} P^{\prime}$ & \\
\hline$i A c j$ & $i A c j$ & $i$ & $i A c j$ & \\
\hline$\downarrow$ & $j A c k$ & $\downarrow$ & $\downarrow$ & \\
\hline \multirow[t]{3}{*}{$i r j$} & $\downarrow$ & $i A c j$ & jAck & \\
\hline & jrk & $i r j$ & jrk & \\
\hline & & $\begin{array}{l}\text { where } j \\
\text { is new }\end{array}$ & $\begin{array}{c}\text { where } k \\
\text { is new }\end{array}$ & \\
\hline$T-a b 4$ & $T-a b 5$ & $T-\mathcal{A} M P$ & $T-\mathcal{W} M P$ & $T-M \mathcal{W} P$ \\
\hline$i r j$ & $i r j$ & $i A c j$ & $i r j$ & $i A c j$ \\
\hline jAck & iAck & irk & jAck & jrk \\
\hline$\downarrow$ & $\downarrow$ & $\downarrow$ & $\downarrow$ & $\downarrow$ \\
\hline \multirow[t]{4}{*}{ iAck } & jAck & $j r l$ & $i A c l$ & irl \\
\hline & & $k A c l$ & lrk & lAck \\
\hline & & where $l$ & where $l$ & where $l$ \\
\hline & & is new & is new & is new \\
\hline
\end{tabular}

\subsubsection{Alethic-doxastic accessibility rules (ad-rules)}

\begin{tabular}{|c|c|c|c|c|}
\hline$T-M \mathcal{B}$ & $T-M \mathcal{B}^{\prime}$ & $T-\mathcal{B P}$ & $T-\mathcal{B} P^{\prime}$ & $T-\mathcal{B} M$ \\
\hline$i D c j$ & $i D c j$ & $i$ & $i D c j$ & $i r j$ \\
\hline$\downarrow$ & jDck & $\downarrow$ & $\downarrow$ & $\downarrow$ \\
\hline \multirow[t]{2}{*}{$i r j$} & $\downarrow$ & $i D c j$ & jDck & $i D c j$ \\
\hline & jrk & $\begin{array}{c}\text { irj } \\
\text { where } j \\
\text { is new }\end{array}$ & $\begin{array}{c}j r k \\
\text { where } k \\
\text { is new }\end{array}$ & \\
\hline$T-a d 4$ & $T-a d 5$ & $T-\mathcal{C} M P$ & $T-\mathcal{B} M P$ & $T-M \mathcal{B} P$ \\
\hline$i r j$ & $i r j$ & $i D c j$ & $i r j$ & $i D c j$ \\
\hline jDck & $i D c k$ & irk & jDck & jrk \\
\hline$\downarrow$ & $\downarrow$ & $\downarrow$ & $\downarrow$ & $\downarrow$ \\
\hline \multirow[t]{4}{*}{$i D c k$} & $j D c k$ & jrl & $i D c l$ & irl \\
\hline & & $k D c l$ & lrk & $l D c k$ \\
\hline & & where $l$ & where $l$ & where $l$ \\
\hline & & is new & is new & is new \\
\hline
\end{tabular}

Australasian Journal of Logic (16:3) 2019, Article no. 3 
4.2.11 Boulesic-doxastic accessibility rules (b-d-rules)

\begin{tabular}{c|c|c|c|c|c}
\hline$T-\mathcal{W B}$ & $T-\mathcal{B W}$ & $T-\mathcal{W C}$ & $T-\mathcal{B A}$ & $T-d b 4$ & $T-d b 5$ \\
\hline$i D c j$ & $i A c j$ & $i$ & $i$ & $i D c j$ & $i D c j$ \\
$\downarrow$ & $\downarrow$ & $\downarrow$ & $\downarrow$ & $j A c k$ & $i A c k$ \\
$i A c j$ & $i D c j$ & $i A c j$ & $i D c j$ & $\downarrow$ & $\downarrow$ \\
& & $i D c j$ & $i A c j$ & $i A c k$ & $j A c k$ \\
& & where $j$ & where $j$ & & \\
\hline$T-b d 4$ & $T-b d 5$ & $T-\mathcal{A B} P$ & $T-\mathcal{C} \mathcal{W} P$ & $T-\mathcal{B} \mathcal{W} P$ & $T-\mathcal{W B P} P$ \\
\hline$i A c j$ & $i A c j$ & $i D c j$ & $i A c j$ & $i A c j$ & $i D c j$ \\
$j D c k$ & $i D c k$ & $i A c k$ & $i D c k$ & $j D c k$ & $j A c k$ \\
$\downarrow$ & $\downarrow$ & $\downarrow$ & $\downarrow$ & $\downarrow$ & $\downarrow$ \\
$i D c k$ & $j D c k$ & $j A c l$ & $j D c l$ & $i D c l$ & $i A c l$ \\
& & $k D c l$ & $k A c l$ & $l A c k$ & $l D c k$ \\
& & where $l$ & where $l$ & where $l$ & where $l$ \\
& & is new & is new & is new & is new \\
\hline$T-\mathcal{B} \mathcal{W B}$ & $T-\mathcal{W B \mathcal { B }}$ & $T-\mathcal{W} \mathcal{W B}$ & $T-\mathcal{B B} \mathcal{W}$ & $T-\mathcal{W} O$ & \\
\hline$i D c j$ & $i A c j$ & $i A c j$ & $i D c j$ & $i A c j$ & \\
$\downarrow$ & $\downarrow$ & $j D c k$ & $j A c k$ & $j D c k$ & \\
$j A c j$ & $j D c j$ & $\downarrow$ & $\downarrow$ & $\downarrow$ & \\
& & $i A c k$ & $i D c k$ & $j=k$ & \\
\hline
\end{tabular}

Table 18

$T-\mathcal{W C}$ is equivalent to $T-\mathcal{B A}$ and $T-\mathcal{A B P}$ is equivalent to $T-\mathcal{C W} P$ (Table 18). Everything that can be proved with $T-\mathcal{W C}$ can be proved with $T-\mathcal{B A}$ and vice versa, and similarly for $T-\mathcal{A B} P$ and $T-\mathcal{C W} P$.

\subsubsection{Alethic-boulesic-doxastic rules (abd rules)}

\begin{tabular}{c|c|c|c|c}
\hline$T-W N I$ & $T-W N O$ & $T-W B P$ & $T-B W P$ & $T-B M W$ \\
\hline$i A c j$ & $i A c j$ & $i A c j$ & $i D c j$ & $i D c j$ \\
$j r k$ & $j D c k$ & $\downarrow$ & $\downarrow$ & $j A c k$ \\
$\downarrow$ & $\downarrow$ & $j D c k$ & $j A c k$ & $\downarrow$ \\
$j D c k$ & $j r k$ & $j r k$ & $j r k$ & $j r k$ \\
& & where $k$ is new & where $k$ is new & \\
\hline
\end{tabular}

Table 19

Australasian Journal of Logic (16:3) 2019, Article no. 3 


\subsubsection{Identity rules}

\begin{tabular}{c|c|c|c|c|c|c}
\hline$T-R=$ & $T-S=$ & $T-N=$, & $T-A=$ & $T-D=$ & $T-I d I$ & $T-I d I I$ \\
$*$ & $s=t, i$ & $a=b, i$ & $a=b, i$ & $a=b, i$ & $\alpha(i)$ & $\alpha(i)$ \\
$\downarrow$ & $A[s / x], i$ & $\downarrow$ & $j A a k$ & $j D a k$ & $i=j$ & $j=i$ \\
$t=t, i$ & $\downarrow$ & $a=b, j$ & $\downarrow$ & $\downarrow$ & $\downarrow$ & $\downarrow$ \\
for every $t$ & $A[t / x], i$ & for any $j$ & $j A b k$ & $j D b k$ & $\alpha(j)$ & $\alpha(j)$ \\
on the branch & & & & & & \\
\hline
\end{tabular}

Table 20

There are two world identity rules: $T-I d I$ and $T-I d I I$ (both abbreviated $I d$ ). $\alpha(i)$ is a line in a tableau that includes ' $i$ ', and $\alpha(j)$ is like $\alpha(i)$ except that ' $i$ ' is replaced by ' $j$ '. That is, if $\alpha(i)$ is $A$, $i$, then $\alpha(j)$ is $A$, $j$; if $\alpha(i)$ is $k r i$, then $\alpha(j)$ is $k r j$; if $\alpha(i)$ is $i=k$, then $\alpha(j)$ is $j=k$, etc. If $\alpha(i)$ is $A, i$ we only apply the rule when $A$ is atomic or of the form $\mathcal{W}_{s} D, \mathcal{A}_{s} D, \mathcal{B}_{s} D$ or $\mathcal{C}_{s} D$ given that $\neg R s, i$ is on the branch.

( $T-S=$ ) is applied only 'within worlds', and we usually only apply the rule when $A$ is atomic. However, we shall also allow applications of the following kind. Let $M$ be a matrix where $x_{m}$ is the first free variable in $M$ and $a_{m}$ is the constant in $M\left[a_{1}, \ldots, a, \ldots, a_{n} / \vec{x}\right]$ that replaces $x_{m}$. Furthermore, suppose we have $a=b, i$, $M\left[a_{1}, \ldots, a, \ldots, a_{n} \mid \vec{x}\right], i$ and $\neg R a_{m}, i$ on the branch. Then we may apply $(T-S=)$ to obtain an extension of the branch that includes $M\left[a_{1}, \ldots, b, \ldots, a_{n} / \vec{x}\right], i$.

\subsubsection{Propositional quantifiers}

\begin{tabular}{c|c|c|c}
\hline$P \Pi$ & $P \Sigma$ & $P \neg \Pi$ & $P \neg \Sigma$ \\
\hline$\Pi X A, i$ & $\Sigma X A, i$ & $\neg \prod X A, i$ & $\neg \Sigma X A, i$ \\
$\downarrow$ & $\downarrow$ & $\downarrow$ & $\downarrow$ \\
$A[B / X], i$ & $A[Y / X], i$ & $\Sigma X \neg A, i$ & $\prod X \neg A, i$ \\
for any $B$ & $\begin{array}{c}\text { where } Y \text { is new } \\
\text { to the branch }\end{array}$ & & \\
\hline
\end{tabular}

Table 21

The propositional quantifiers are similar to the possibilist quantifiers. Nonetheless, in these rules $X, Y$ and $B$ do not refer to individuals; $X$ and $Y$ are propositional variables and $B$ is a formula, with the usual provisos (see Section 3.2). ' $P$ ' in ' $P \Pi$ ' stands for 'propositional'. We can omit the initial ' $P$ ' if it is clear from the context that we are talking about the propositional quantifiers. 


\subsection{Tableau systems and some basic proof-theoretical concepts}

A tableau system is a set of tableau rules. A boulesic-doxastic (tableau) system (or logic) is a tableau system that includes the propositional rules, the basic alethic, boulesic and doxastic rules, the rules for the possibilist quantifiers, the $C U T$-rule and the identity rules. The smallest boulesic-doxastic system is called $\mathcal{B D}$. By adding various tableau rules to $\mathcal{B D}$, we obtain a large class of stronger boulesicdoxastic systems, and by adding the rules for the propositional quantifiers, we can generate a set of 'augmented systems'. A non-augmented system is based on the language $\mathcal{L}$ and an augmented system is based on $\mathcal{L}_{S u b}$.

Among all the many boulesic-doxastic systems, which system is the correct one? I do not think that this question has a simple answer. Different systems may be useful for different purposes. However, I would like to mention a logic that seems particularly interesting to me. This logic contains every tableau rule in Section 4.2 except $T-d O, T-M \mathcal{W} P, T-\mathcal{W B}, T-\mathcal{B} \mathcal{W B}, T-\mathcal{W} \mathcal{W B}, T-\mathcal{B} \mathcal{W} P, T-\mathcal{W} O$, $T-U R$ and the propositional quantifiers. Let us call this system strong boulesicdoxastic logic. By adding the propositional quantifiers we obtain augmented strong boulesic-doxastic logic. Strong boulesic-doxastic logic includes many redundant rules and there are many systems that are deductively equivalent that contain fewer primitive rules. It is beyond the scope of this paper to try to argue for this system and defend it against various criticisms. It is possible to derive some surprising and perhaps prima facie counterintuitive theorems in it, but it nevertheless seems quite attractive to me.

Important proof theoretical concepts like the concepts of proof, theorem, derivation, consistency, inconsistency in a system, the logic of a tableau system, etc. are defined in a standard way (for more on the tableau method, see the references in the introduction).

\section{Examples of theorems}

In this section, I will present some sentences that can be proved in various systems. The informal reading of the theses should be obvious. A 'system' means a 'boulesic-doxastic system' and a ' $T$-system' means a 'boulesic-doxastic system that includes $T$ '.

Furthermore, I use the following abbreviations. We have omitted the initial ' $T$-' in the names of the rules. $T_{1} \cdot T_{2}$ stands for a system that includes both $T_{1}$ and $T_{2}$, where $T_{1}$ and $T_{2}$ are some non-basic tableau rules. $T_{1}: T_{2}$ stands for a system that includes $T_{1}$ or $T_{2}$. For example, consider Table 27. $\mathcal{A B P}$. $(U R$ : $(M \mathcal{B} . M \mathcal{W} . F T R))$ means that the theorem $\mathcal{A B P}=\Pi x\left(R x \rightarrow\left(\mathcal{A}_{x} \mathcal{B}_{x} A \rightarrow \mathcal{B}_{x} \mathcal{A}_{x} A\right)\right)$ is provable in every logic that includes the tableau rules $\mathcal{A B P}$, and $U R$ or $M \mathcal{B}, M \mathcal{W}$ 
and FTR. In other words, this sentence is a theorem in every system that includes $\mathcal{A B P}$ and $U R$, and it is a theorem in every system that includes $\mathcal{A B P}, M \mathcal{B}, M \mathcal{W}$ and FTR. $\mathcal{A B P}$ is also a theorem in every logic that includes the tableau rules $\mathcal{C W} P$, and $U R$ or $M \mathcal{B}, M \mathcal{W}$ and $F T R$.

\begin{tabular}{l|l}
\hline Theorem & Systems \\
\hline$\Pi x\left(R x \rightarrow\left(\mathcal{W}_{x} B \leftrightarrow \neg \mathcal{A}_{x} \neg B\right)\right)$ & Every \\
$\Pi x\left(R x \rightarrow\left(\neg \mathcal{W}_{x} B \leftrightarrow \mathcal{A}_{x} \neg B\right)\right)$ & Every \\
$\Pi x\left(R x \rightarrow\left(\mathcal{W}_{x} \neg B \leftrightarrow \neg \mathcal{A}_{x} B\right)\right)$ & Every \\
$\Pi x\left(R x \rightarrow\left(\mathcal{A}_{x} B \leftrightarrow \neg \mathcal{W}_{x} \neg B\right)\right)$ & Every \\
$\Pi x\left(R x \rightarrow\left(\mathcal{W}_{x}(A \wedge B) \leftrightarrow\left(\mathcal{W}_{x} A \wedge \mathcal{W}_{x} B\right)\right)\right)$ & Every \\
$\Pi x\left(R x \rightarrow\left(\left(\mathcal{W}_{x} A \vee \mathcal{W}_{x} B\right) \rightarrow \mathcal{W}_{x}(A \vee B)\right)\right)$ & Every \\
$\Pi x\left(R x \rightarrow\left(\mathcal{A}_{x}(A \wedge B) \rightarrow\left(\mathcal{A}_{x} A \wedge \mathcal{A}_{x} B\right)\right)\right)$ & Every \\
$\Pi x\left(R x \rightarrow\left(\mathcal{A}_{x}(A \vee B) \leftrightarrow\left(\mathcal{A}_{x} A \vee \mathcal{A}_{x} B\right)\right)\right)$ & Every \\
$\Pi x\left(R x \rightarrow\left(\mathcal{W}_{x}(A \rightarrow B) \rightarrow\left(\mathcal{W}_{x} A \rightarrow \mathcal{W}_{x} B\right)\right)\right)$ & Every \\
$\Pi x\left(R x \rightarrow\left(\mathcal{W}_{x}(A \rightarrow B) \rightarrow\left(\mathcal{A}_{x} A \rightarrow \mathcal{A}_{x} B\right)\right)\right)$ & Every \\
$\Pi x\left(R x \rightarrow\left(\mathcal{W}_{x}(A \rightarrow B) \rightarrow\left(\mathcal{W}_{x} \neg B \rightarrow \mathcal{W}_{x} \neg A\right)\right)\right)$ & Every \\
$\Pi x\left(R x \rightarrow\left(\mathcal{W}_{x}(A \rightarrow B) \rightarrow\left(\neg \mathcal{W}_{x} B \rightarrow \neg \mathcal{W}_{x} A\right)\right)\right)$ & Every \\
$\Pi x\left(R x \rightarrow\left(\mathcal{W}_{x}(A \leftrightarrow B) \rightarrow\left(\mathcal{W}_{x} A \leftrightarrow \mathcal{W}_{x} B\right)\right)\right)$ & Every \\
$\Pi x\left(R x \rightarrow\left(\mathcal{W}_{x}(A \leftrightarrow B) \rightarrow\left(\mathcal{A}_{x} A \leftrightarrow \mathcal{A}_{x} B\right)\right)\right)$ & Every \\
$\Pi x\left(R x \rightarrow\left(\mathcal{W}_{x}(A \leftrightarrow B) \rightarrow\left(\mathcal{W}_{x} \neg A \leftrightarrow \mathcal{W}_{x} \neg B\right)\right)\right)$ & Every \\
$\Pi x\left(R x \rightarrow\left(\mathcal{W}_{x}(A \leftrightarrow B) \rightarrow\left(\neg \mathcal{W}_{x} A \leftrightarrow \neg \mathcal{W}_{x} B\right)\right)\right)$ & Every \\
\hline
\end{tabular}

Table 22 (Some pure boulesic sentences that are theorems in every system)

Let $A$ be a theorem in Table 22 and let $T(A)$ be the sentence that is obtained from $A$ by replacing every occurrence of $\mathcal{W}$ by $\mathcal{B}$ and every occurrence of $\mathcal{A}$ by $\mathcal{C}$. Then $T(A)$ is a theorem in every system.

\begin{tabular}{l|l|l}
\hline Name & Theorem & Systems \\
\hline$b D$ & $\Pi x\left(R x \rightarrow \neg\left(\mathcal{W}_{x} B \wedge \mathcal{W}_{x} \neg B\right)\right)$ & $b D$ \\
$b 4$ & $\Pi x\left(R x \rightarrow\left(\mathcal{W}_{x} B \rightarrow \mathcal{W}_{x} \mathcal{W}_{x} B\right)\right)$ & $b 4 .(U R:(M \mathcal{W} . F T R))$ \\
$b 5$ & $\Pi x\left(R x \rightarrow\left(\mathcal{A}_{x} B \rightarrow \mathcal{W}_{x} \mathcal{A}_{x} B\right)\right)$ & $b 5 .(U R:(M \mathcal{W} . F T R))$ \\
$b T^{\prime}$ & $\Pi x\left(R x \rightarrow \mathcal{W}_{x}\left(\mathcal{W}_{x} B \rightarrow B\right)\right)$ & $b T^{\prime} .(U R:(M \mathcal{W} . F T R))$ \\
$b B^{\prime}$ & $\Pi x\left(R x \rightarrow \mathcal{W}_{x}\left(\mathcal{A}_{x} \mathcal{W}_{x} B \rightarrow B\right)\right)$ & $b B^{\prime} .(U R:(M \mathcal{W} . F T R))$ \\
\hline
\end{tabular}

Table 23 (Some pure boulesic sentences that are theorems in various systems) 


\begin{tabular}{|c|c|c|}
\hline Name & Theorem & Systems \\
\hline$d O$ & $\Pi x\left(R x \rightarrow\left(A \rightarrow \mathcal{B}_{x} A\right)\right)$ & $d O$ \\
\hline$d T$ & $\Pi x\left(R x \rightarrow\left(\mathcal{B}_{x} A \rightarrow A\right)\right)$ & $d T$ \\
\hline$d D$ & $\Pi x\left(R x \rightarrow \neg\left(\mathcal{B}_{x} D \wedge \mathcal{B}_{x} \neg D\right)\right)$ & $d D$ \\
\hline$d B$ & $\Pi x\left(R x \rightarrow\left(D \rightarrow \mathcal{B}_{x} \mathcal{C}_{x} D\right)\right)$ & dB. $(U R:(M \mathcal{B} . F T R))$ \\
\hline$d 4$ & $\Pi x\left(R x \rightarrow\left(\mathcal{B}_{x} D \rightarrow \mathcal{B}_{x} \mathcal{B}_{x} D\right)\right)$ & d4. $(U R:(M \mathcal{B} . F T R))$ \\
\hline$d 5$ & $\Pi x\left(R x \rightarrow\left(\mathcal{C}_{x} D \rightarrow \mathcal{B}_{x} \mathcal{C}_{x} D\right)\right)$ & d5. (UR: $(M \mathcal{B} . F T R))$ \\
\hline$d T^{\prime}$ & $\Pi x\left(R x \rightarrow \mathcal{B}_{x}\left(\mathcal{B}_{x} D \rightarrow D\right)\right)$ & $d T^{\prime} .(U R:(M \mathcal{B} . F T R))$ \\
\hline$d B^{\prime}$ & $\Pi x\left(R x \rightarrow \mathcal{B}_{x}\left(\mathcal{C}_{x} \mathcal{B}_{x} D \rightarrow D\right)\right)$ & $d B^{\prime} .(U R:(M \mathcal{B} . F T R))$ \\
\hline
\end{tabular}

Table 24 (Some pure doxastic sentences that are theorems in various systems)

\begin{tabular}{l|l|l}
\hline Name & Theorem & Systems \\
\hline$M \mathcal{W}$ & $\Pi x\left(R x \rightarrow\left(\square A \rightarrow \mathcal{W}_{x} A\right)\right)$ & $M \mathcal{W}$ \\
$\mathcal{W} P$ & $\Pi x\left(R x \rightarrow\left(\mathcal{W}_{x} A \rightarrow \diamond A\right)\right)$ & $\mathcal{W} C$ \\
$M \mathcal{W}^{\prime}$ & $\Pi x\left(R x \rightarrow \mathcal{W}_{x}\left(\square A \rightarrow \mathcal{W}_{x} A\right)\right)$ & $M \mathcal{W}^{\prime} . U R$ \\
$\mathcal{W} P^{\prime}$ & $\Pi x\left(R x \rightarrow \mathcal{W}_{x}\left(\mathcal{W}_{x} A \rightarrow \diamond A\right)\right)$ & $\mathcal{W} C^{\prime} .(U R:(M \mathcal{W} . F T R))$ \\
$a b 4$ & $\Pi x\left(R x \rightarrow\left(\mathcal{W}_{x} A \rightarrow \square \mathcal{W}_{x} A\right)\right)$ & $a b 4 .(U R: F T R)$ \\
$a b 5$ & $\Pi x\left(R x \rightarrow\left(\mathcal{A}_{x} B \rightarrow \square \mathcal{A}_{x} B\right)\right)$ & $a b 5 .(U R: F T R)$ \\
$\mathcal{A M P}$ & $\Pi x\left(R x \rightarrow\left(\mathcal{A}_{x} \square B \rightarrow \square \mathcal{A}_{x} B\right)\right)$ & $\mathcal{A M P .}(U R: F T R)$ \\
$\mathcal{W} M P$ & $\Pi x\left(R x \rightarrow\left(\mathcal{W}_{x} \square A \rightarrow \square \mathcal{W}_{x} A\right)\right)$ & $\mathcal{W} M P .(U R: F T R)$ \\
$M \mathcal{W} P$ & $\Pi x\left(R x \rightarrow\left(\square \mathcal{W}_{x} A \rightarrow \mathcal{W}_{x} \square A\right)\right)$ & $M \mathcal{W} P .(U R: F T R)$ \\
\hline
\end{tabular}

Table 25 (Some alethic-boulesic sentences that are theorems in various systems)

\begin{tabular}{l|l|l}
\hline Name & Theorem & Systems \\
\hline$M \mathcal{B}$ & $\prod x\left(R x \rightarrow\left(\square A \rightarrow \mathcal{B}_{x} A\right)\right)$ & $M \mathcal{B}$ \\
$\mathcal{B P}$ & $\prod x\left(R x \rightarrow\left(\mathcal{B}_{x} A \rightarrow \diamond A\right)\right)$ & $\mathcal{B} C$ \\
$M \mathcal{B}^{\prime}$ & $\prod x\left(R x \rightarrow \mathcal{B}_{x}\left(\square A \rightarrow \mathcal{B}_{x} A\right)\right)$ & $M \mathcal{B}^{\prime} . U R$ \\
$\mathcal{B} P^{\prime}$ & $\prod x\left(R x \rightarrow \mathcal{B}_{x}\left(\mathcal{B}_{x} A \rightarrow \diamond A\right)\right)$ & $\mathcal{B} C^{\prime} .($ UR:(MB.FTR $\left.)\right)$ \\
$\mathcal{B} M$ & $\prod x\left(R x \rightarrow\left(\mathcal{B}_{x} A \rightarrow \square A\right)\right)$ & $\mathcal{B} M$ \\
$a d 4$ & $\prod x\left(R x \rightarrow\left(\mathcal{B}_{x} A \rightarrow \square \mathcal{B}_{x} A\right)\right)$ & $a d 4 .(U R: F T R)$ \\
$a d 5$ & $\prod x\left(R x \rightarrow\left(\mathcal{C}_{x} B \rightarrow \square \mathcal{C}_{x} B\right)\right)$ & $a d 5 .(U R: F T R)$ \\
$\mathcal{C} M P$ & $\prod x\left(R x \rightarrow\left(\mathcal{C}_{x} \square B \rightarrow \square \mathcal{C}_{x} B\right)\right)$ & $\mathcal{C} M P .(U R: F T R)$ \\
$\mathcal{B} M P$ & $\prod x\left(R x \rightarrow\left(\mathcal{B}_{x} \square A \rightarrow \square \mathcal{B}_{x} A\right)\right)$ & $\mathcal{B} M P .(U R: F T R)$ \\
$M \mathcal{B} P$ & $\prod x\left(R x \rightarrow\left(\square \mathcal{B}_{x} A \rightarrow \mathcal{B}_{x} \square A\right)\right)$ & $M \mathcal{B P} .(U R: F T R)$ \\
\hline
\end{tabular}

Table $\overline{26 \text { (Some alethic-doxastic sentences that are theorems in various systems) }}$ 


\begin{tabular}{|c|c|c|}
\hline Name & Theorem & Systems \\
\hline $\mathcal{W B}$ & $\Pi x\left(R x \rightarrow\left(\mathcal{W}_{x} A \rightarrow \mathcal{B}_{x} A\right)\right)$ & $\mathcal{W B}$ \\
\hline $\mathcal{B W}$ & $\Pi x\left(R x \rightarrow\left(\mathcal{B}_{x} A \rightarrow \mathcal{W}_{x} A\right)\right)$ & $\mathcal{B W}$ \\
\hline $\mathcal{W C}$ & $\Pi x\left(R x \rightarrow\left(\mathcal{W}_{x} A \rightarrow \mathcal{C}_{x} A\right)\right)$ & $\mathcal{W C}: \mathcal{B A}$ \\
\hline $\mathcal{B A}$ & $\Pi x\left(R x \rightarrow\left(\mathcal{B}_{x} A \rightarrow \mathcal{A}_{x} A\right)\right)$ & $\mathcal{B A}: \mathcal{W C}$ \\
\hline $\mathcal{B W B}$ & $\Pi x\left(R x \rightarrow\left(\mathcal{B}_{x} \mathcal{W}_{x} A \rightarrow \mathcal{B}_{x} A\right)\right)$ & BWB. $(U R:(M \mathcal{B} . F T R))$ \\
\hline $\mathcal{W B W}$ & $\Pi x\left(R x \rightarrow\left(\mathcal{W}_{x} \mathcal{B}_{x} A \rightarrow \mathcal{W}_{x} A\right)\right)$ & $\mathcal{W B W} \cdot(U R:(M \mathcal{W} . F T R))$ \\
\hline $\mathcal{W I}$ & $\Pi x\left(R x \rightarrow \mathcal{W}_{x}\left(\mathcal{B}_{x} A \rightarrow A\right)\right)$ & $\mathcal{W B W} \cdot(U R:(M \mathcal{W} . F T R))$ \\
\hline$d b 4$ & $\Pi x\left(R x \rightarrow\left(\mathcal{W}_{x} A \rightarrow \mathcal{B}_{x} \mathcal{W}_{x} A\right)\right)$ & $d b 4 .(U R:(M \mathcal{B} . F T R))$ \\
\hline$d b 5$ & $\Pi x\left(R x \rightarrow\left(\mathcal{A}_{x} A \rightarrow \mathcal{B}_{x} \mathcal{A}_{x} A\right)\right)$ & db5.(UR: (MB.FTR $))$ \\
\hline$b d 4$ & $\Pi x\left(R x \rightarrow\left(\mathcal{B}_{x} A \rightarrow \mathcal{W}_{x} \mathcal{B}_{x} A\right)\right)$ & bd4. $(U R:(M \mathcal{W} . F T R))$ \\
\hline$b d 5$ & $\Pi x\left(R x \rightarrow\left(\mathcal{C}_{x} A \rightarrow \mathcal{W}_{x} \mathcal{C}_{x} A\right)\right)$ & bd5.(UR: $(M \mathcal{W} . F T R))$ \\
\hline $\mathcal{W W B}$ & $\Pi x\left(R x \rightarrow\left(\mathcal{W}_{x} A \rightarrow \mathcal{W}_{x} \mathcal{B}_{x} A\right)\right)$ & WWWB. $(U R:(M \mathcal{W} . F T R))$ \\
\hline $\mathcal{B B W}$ & $\Pi x\left(R x \rightarrow\left(\mathcal{B}_{x} A \rightarrow \mathcal{B}_{x} \mathcal{W}_{x} A\right)\right)$ & $\mathcal{B B W} \cdot(U R:(M \mathcal{B} . F T R))$ \\
\hline $\mathcal{A B P}$ & $\Pi x\left(R x \rightarrow\left(\mathcal{A}_{x} \mathcal{B}_{x} A \rightarrow \mathcal{B}_{x} \mathcal{A}_{x} A\right)\right)$ & $\begin{array}{l}\mathcal{A B P} .(U R:(M \mathcal{B} . M \mathcal{W} . F T R)) \\
\mathcal{C} \mathcal{W} P .(U R:(M \mathcal{B} . M \mathcal{W} . F T R))\end{array}$ \\
\hline $\mathcal{C W P}$ & $\Pi x\left(R x \rightarrow\left(\mathcal{C}_{x} \mathcal{W}_{x} A \rightarrow \mathcal{W}_{x} \mathcal{C}_{x} A\right)\right)$ & $\begin{array}{l}\mathcal{C W} P .(U R:(M \mathcal{B} . M \mathcal{W} . F T R)) \\
\mathcal{A B P} .(U R:(M \mathcal{B} . M \mathcal{W} . F T R))\end{array}$ \\
\hline $\mathcal{B W P}$ & $\Pi x\left(R x \rightarrow\left(\mathcal{B}_{x} \mathcal{W}_{x} A \rightarrow \mathcal{W}_{x} \mathcal{B}_{x} A\right)\right)$ & $\mathcal{B} \mathcal{W} P .(U R:(M \mathcal{B} . M \mathcal{W} . F T R))$ \\
\hline $\mathcal{W B P}$ & $\Pi x\left(R x \rightarrow\left(\mathcal{W}_{x} \mathcal{B}_{x} A \rightarrow \mathcal{B}_{x} \mathcal{W}_{x} A\right)\right)$ & WBP. $(U R:(M \mathcal{B} . M \mathcal{W} . F T R))$ \\
\hline $\mathcal{W O}$ & $\Pi x\left(R x \rightarrow \mathcal{W}_{x}\left(A \rightarrow \mathcal{B}_{x} A\right)\right)$ & $\mathcal{W} O .(U R:(M \mathcal{W} . F T R))$ \\
\hline
\end{tabular}

Table 27 (Some boulesic-doxastic sentences that are theorems in various systems) 
Consider $d b 4$. This is a formalisation of (i) in Example 2 (see the introduction). I will now show that this formula is a theorem in every system that includes $T-d b 4$, $T-M \mathcal{B}$ and $T-F T R$. This will illustrate how to use semantic tableaux. To prove that a sentence $A$ is a theorem in some system $S$, we construct a closed semantic $S$-tableau that starts with $\neg A, 0$. An $S$-tableau is a tableau that has been generated in accordance with the rules in $S$.

$$
\begin{gathered}
(1) \neg \prod x\left(R x \rightarrow\left(\mathcal{W}_{x} A \rightarrow \mathcal{B}_{x} \mathcal{W}_{x} A\right)\right), 0 \\
(2) \Sigma x \neg\left(R x \rightarrow\left(\mathcal{W}_{x} A \rightarrow \mathcal{B}_{x} \mathcal{W}_{x} A\right)\right), 0[1, \neg \Pi] \\
(3) \neg\left(R c \rightarrow\left(\mathcal{W}_{c} A \rightarrow \mathcal{B}_{c} \mathcal{W}_{c} A\right)\right), 0[2, \Sigma] \\
(4) R c, 0[3, \neg \rightarrow] \\
(5) \neg\left(\mathcal{W}_{c} A \rightarrow \mathcal{B}_{c} \mathcal{W}_{c} A\right)[3, \neg \rightarrow] \\
(6) \mathcal{W}_{c} A, 0[5, \neg \rightarrow] \\
(7) \neg \mathcal{B}_{c} \mathcal{W}_{c} A, 0[5, \neg \rightarrow] \\
(8) \mathcal{C}_{c} \neg \mathcal{W}_{c} A, 0[4,7, \neg \mathcal{B}] \\
\text { (9) } 0 D c 1[4,8, \mathcal{C}] \\
(10) \neg \mathcal{W}_{c} A, 1[4,8, \mathcal{C}] \\
\text { (11) } 0 r 1[9, T-M \mathcal{B}] \\
(12) \operatorname{Rc}, 1[4,11, T-F T R] \\
\text { (13) } \mathcal{A}_{c} \neg A, 1[10,12, \neg \mathcal{W}] \\
\text { (14) } 1 A c 2[12,13, \mathcal{A}] \\
\text { (15) } \neg A, 2[12,13, \mathcal{A}] \\
\text { (16) } 0 A c 2[9,14, T-d b 4] \\
\text { (17) } A, 2[4,6,16, \mathcal{W}] \\
(18) *[15,17]
\end{gathered}
$$

\begin{tabular}{l|l}
\hline Theorem & Systems \\
\hline$\Pi x\left(R x \rightarrow\left(\Pi y \mathcal{W}_{x} D \leftrightarrow \mathcal{W}_{x} \Pi y D\right)\right)$ & Every \\
$\Pi x\left(R x \rightarrow\left(\Sigma y \mathcal{A}_{x} D \leftrightarrow \mathcal{A}_{x} \Sigma y D\right)\right)$ & Every \\
$\Pi x\left(R x \rightarrow\left(\mathcal{A}_{x} \Pi y D \rightarrow \Pi y \mathcal{A}_{x} D\right)\right)$ & Every \\
$\Pi x\left(R x \rightarrow\left(\Sigma y \mathcal{W}_{x} D \rightarrow \mathcal{W}_{x} \Sigma y D\right)\right)$ & Every \\
$\Pi x\left(R x \rightarrow\left(\Pi y \mathcal{B}_{x} D \leftrightarrow \mathcal{B}_{x} \Pi y D\right)\right)$ & Every \\
$\Pi x\left(R x \rightarrow\left(\Sigma y \mathcal{C}_{x} D \leftrightarrow \mathcal{C}_{x} \Sigma y D\right)\right)$ & Every \\
$\Pi x\left(R x \rightarrow\left(\mathcal{C}_{x} \Pi y D \rightarrow \Pi y \mathcal{C}_{x} D\right)\right)$ & Every \\
$\Pi x\left(R x \rightarrow\left(\Sigma y \mathcal{B}_{x} D \rightarrow \mathcal{B}_{x} \Sigma y D\right)\right)$ & Every \\
\hline
\end{tabular}

Table 28 (Some Barcan-like sentences that are theorems in every system) 


\begin{tabular}{l|l}
\hline Theorem & Systems \\
\hline$\Pi x\left(R x \rightarrow\left(\square(A \rightarrow B) \rightarrow\left(\mathcal{W}_{x} A \rightarrow \mathcal{W}_{x} B\right)\right)\right)$ & $M \mathcal{W}$ \\
$\Pi x\left(R x \rightarrow\left(\square(A \rightarrow B) \rightarrow\left(\mathcal{A}_{x} A \rightarrow \mathcal{A}_{x} B\right)\right)\right)$ & $M \mathcal{W}$ \\
$\Pi x\left(R x \rightarrow\left(\square(A \rightarrow B) \rightarrow\left(\mathcal{W}_{x} \neg B \rightarrow \mathcal{W}_{x} \neg A\right)\right)\right)$ & $M \mathcal{W}$ \\
$\Pi x\left(R x \rightarrow\left(\square(A \rightarrow B) \rightarrow\left(\neg \mathcal{W}_{x} B \rightarrow \neg \mathcal{W}_{x} A\right)\right)\right)$ & $M \mathcal{W}$ \\
$\Pi x\left(R x \rightarrow\left(\square(A \leftrightarrow B) \rightarrow\left(\mathcal{W}_{x} A \leftrightarrow \mathcal{W}_{x} B\right)\right)\right)$ & $M \mathcal{W}$ \\
$\Pi x\left(R x \rightarrow\left(\square(A \leftrightarrow B) \rightarrow\left(\mathcal{A}_{x} A \leftrightarrow \mathcal{A}_{x} B\right)\right)\right)$ & $M \mathcal{W}$ \\
$\Pi x\left(R x \rightarrow\left(\square(A \leftrightarrow B) \rightarrow\left(\mathcal{W}_{x} \neg A \leftrightarrow \mathcal{W}_{x} \neg B\right)\right)\right)$ & $M \mathcal{W}$ \\
$\Pi x\left(R x \rightarrow\left(\square(A \leftrightarrow B) \rightarrow\left(\neg \mathcal{W}_{x} A \leftrightarrow \neg \mathcal{W}_{x} B\right)\right)\right)$ & $M \mathcal{W}$ \\
\hline
\end{tabular}

Table 29 (Some alethic-boulesic sentences that are theorems in every $M \mathcal{W}$-system)

Let $A$ be a theorem in Table 29 and let $T(A)$ be the sentence that is obtained from $A$ by replacing every occurrence of $\mathcal{W}$ by $\mathcal{B}$ and every occurrence of $\mathcal{A}$ by $\mathcal{C}$. Then $T(A)$ is a theorem in every $M \mathcal{B}$-system.

\begin{tabular}{l|l|l}
\hline Name & Theorem & Systems \\
\hline$W N I$ & $\Pi x\left(R x \rightarrow \mathcal{W}_{x}\left(\mathcal{B}_{x} A \rightarrow \square A\right)\right)$ & WNI. $(U R:(F T R . M \mathcal{W})$ \\
$W N O$ & $\Pi x\left(R x \rightarrow \mathcal{W}_{x}\left(\square A \rightarrow \mathcal{B}_{x} A\right)\right)$ & WNO. $(U R:(F T R . M \mathcal{W})$ \\
$W B P$ & $\Pi x\left(R x \rightarrow \mathcal{W}_{x}\left(\mathcal{B}_{x} A \rightarrow \diamond A\right)\right)$ & WBP. $(U R:(F T R . M \mathcal{W})$ \\
$B W P$ & $\Pi x\left(R x \rightarrow \mathcal{B}_{x}\left(\mathcal{W}_{x} A \rightarrow \diamond A\right)\right)$ & BWC. $(U R:(F T R . M \mathcal{B})$ \\
$B M W$ & $\Pi x\left(R x \rightarrow \mathcal{B}_{x}\left(\square A \rightarrow \mathcal{W}_{x} A\right)\right)$ & BMW. $(U R:(F T R . M \mathcal{B})$ \\
\hline \multicolumn{2}{|c}{ Table 30 (Some alethic-boulesic-doxastic theorems) }
\end{tabular}

\begin{tabular}{l|l|l}
\hline Name & Theorem & Systems \\
\hline$U R$ & $\Pi x(R x \rightarrow U R x)$ & $U R$ \\
& $\Pi x\left(R x \rightarrow \mathcal{W}_{x} R x\right)$ & $U R:(F T R . M \mathcal{W})$ \\
& $\Pi x\left(R x \rightarrow \mathcal{A}_{x} R x\right)$ & $(U R . b D):(F T R . M \mathcal{W} . b D)$ \\
& $\Pi x\left(R x \rightarrow \mathcal{B}_{x} R x\right)$ & UR:(FTR.MB $)$ \\
& $\Pi x\left(R x \rightarrow \mathcal{C}_{x} R x\right)$ & $(U R . d D):(F T R . M \mathcal{B} . d D)$ \\
\hline \multicolumn{3}{|c}{ Table 31 (Some theorems concerning $R)$}
\end{tabular}




\begin{tabular}{l|l|l}
\hline Name & Theorem & Systems \\
\hline & $\left(\mathcal{W}_{c} D \wedge c=d\right) \rightarrow \mathcal{W}_{d} D$ & Every \\
& $\left(\mathcal{A}_{c} D \wedge c=d\right) \rightarrow \mathcal{A}_{d} D$ & Every \\
& $\Pi x \Pi y\left(\left(\mathcal{W}_{x} D \wedge x=y\right) \rightarrow \mathcal{W}_{y} D\right)$ & Every \\
& $\Pi x \Pi y\left(\left(\mathcal{A}_{x} D \wedge x=y\right) \rightarrow \mathcal{A}_{y} D\right)$ & Every \\
& $\left(\mathcal{B}_{c} D \wedge c=d\right) \rightarrow \mathcal{B}_{d} D$ & Every \\
& $\left(\mathcal{C}_{c} D \wedge c=d\right) \rightarrow \mathcal{C}_{d} D$ & Every \\
& $\Pi x \Pi y\left(\left(\mathcal{B}_{x} D \wedge x=y\right) \rightarrow \mathcal{B}_{y} D\right)$ & Every \\
& $\Pi x \Pi y\left(\left(\mathcal{C}_{x} D \wedge x=y\right) \rightarrow \mathcal{C}_{y} D\right)$ & Every \\
& $\Pi x \Pi y(x=y \rightarrow U x=y)$ & Every \\
& $\Pi x \Pi y(x=y \rightarrow \square x=y)$ & Every \\
& $\Pi x \Pi y(\neg x=y \rightarrow U \neg x=y)$ & Every \\
& $\Pi x \Pi y(\neg x=y \rightarrow \square \neg x=y)$ & Every \\
\hline
\end{tabular}

Table 32 (Some theorems that include the identity sign)

\begin{tabular}{l|l|l|l}
\hline 1 & 2 & 3 & 4 \\
\hline$(a) P B S$ & $(a) N B S$ & $(a) P B I$ & $(a) N B I$ \\
$\mathcal{B}_{c} D \rightarrow \mathcal{B}_{c} \mathcal{B}_{c} D$ & $\neg \mathcal{B}_{c} D \rightarrow \mathcal{B}_{c} \neg \mathcal{B}_{c} D$ & $\mathcal{B}_{c} \mathcal{B}_{c} D \rightarrow \mathcal{B}_{c} D$ & $\mathcal{B}_{c} \neg \mathcal{B}_{c} D \rightarrow \neg \mathcal{B}_{c} D$ \\
\hline$(b) N M S$ & $(b) P M S$ & $(b) N M I$ & $(b) P M I$ \\
$\neg \mathcal{C}_{c} D \rightarrow \mathcal{B}_{c} \neg \mathcal{C}_{c} D$ & $\mathcal{C}_{c} D \rightarrow \mathcal{B}_{c} \mathcal{C}_{c} D$ & $\mathcal{B}_{c} \neg \mathcal{C}_{c} D \rightarrow \neg \mathcal{C}_{c} D$ & $\mathcal{B}_{c} \mathcal{C}_{c} D \rightarrow \mathcal{C}_{c} D$ \\
\hline$(c) P M C$ & $(c) P B C$ & $(c) P M M$ & $(c) N M M$ \\
$\mathcal{C}_{c} \mathcal{C}_{c} D \rightarrow \mathcal{C}_{c} D$ & $\mathcal{C}_{c} \mathcal{B}_{c} D \rightarrow \mathcal{B}_{c} D$ & $\mathcal{C}_{c} D \rightarrow \mathcal{C}_{c} \mathcal{C}_{c} D$ & $\neg \mathcal{C}_{c} D \rightarrow \mathcal{C}_{c} \neg \mathcal{C}_{c} D$ \\
\hline$(d) N B C$ & $(d) N M C$ & $(d) N B M$ & $(d) P B M$ \\
$\mathcal{C}_{c} \neg \mathcal{B}_{c} D \rightarrow \neg \mathcal{B}_{c} D$ & $\mathcal{C}_{c} \neg \mathcal{C}_{c} D \rightarrow \neg \mathcal{C}_{c} D$ & $\neg \mathcal{B}_{c} D \rightarrow \mathcal{C}_{c} \neg \mathcal{B}_{c} D$ & $\mathcal{B}_{c} D \rightarrow \mathcal{C}_{c} \mathcal{B}_{c} D$ \\
\hline
\end{tabular}

Table 33 (Some boulesic-doxastic principles)

\begin{tabular}{l|l|l|l}
\hline 5 & 6 & 7 & 8 \\
\hline$(a) P W L$ & $(a) N W L$ & $(a) P W G$ & $(a) N W G$ \\
$\mathcal{W}_{c} D \rightarrow \mathcal{W}_{c} \mathcal{W}_{c} D$ & $\neg \mathcal{W}_{c} D \rightarrow \mathcal{W}_{c} \neg \mathcal{W}_{c} D$ & $\mathcal{W}_{c} \mathcal{W}_{c} D \rightarrow \mathcal{W}_{c} D$ & $\mathcal{W}_{c} \neg \mathcal{W}_{c} D \rightarrow \neg \mathcal{W}_{c} D$ \\
\hline$(b) N A L$ & $(b) P A L$ & $(b) N A G$ & $(b) P A G$ \\
$\neg \mathcal{A}_{c} D \rightarrow \mathcal{W}_{c} \neg \mathcal{A}_{c} D$ & $\mathcal{A}_{c} D \rightarrow \mathcal{W}_{c} \mathcal{A}_{c} D$ & $\mathcal{W}_{c} \neg \mathcal{A}_{c} D \rightarrow \neg \mathcal{A}_{c} D$ & $\mathcal{W}_{c} \mathcal{A}_{c} D \rightarrow \mathcal{A}_{c} D$ \\
\hline$(c) P A P$ & $(c) P W P$ & $(c) P A A$ & $(c) N A A$ \\
$\mathcal{A}_{c} \mathcal{A}_{c} D \rightarrow \mathcal{A}_{c} D$ & $\mathcal{A}_{c} \mathcal{W}_{c} D \rightarrow \mathcal{W}_{c} D$ & $\mathcal{A}_{c} D \rightarrow \mathcal{A}_{c} \mathcal{A}_{c} D$ & $\neg \mathcal{A}_{c} D \rightarrow \mathcal{A}_{c} \neg \mathcal{A}_{c} D$ \\
\hline$(d) N W P$ & $(d) N A P$ & $(d) N W A$ & $(d) P W A$ \\
$\mathcal{A}_{c} \neg \mathcal{W}_{c} D \rightarrow \neg \mathcal{W}_{c} D$ & $\mathcal{A}_{c} \neg \mathcal{A}_{c} D \rightarrow \neg \mathcal{A}_{c} D$ & $\neg \mathcal{W}_{c} D \rightarrow \mathcal{A}_{c} \neg \mathcal{W}_{c} D$ & $\mathcal{W}_{c} D \rightarrow \mathcal{A}_{c} \mathcal{W}_{c} D$ \\
\hline
\end{tabular}

Table 34 (Some boulesic-doxastic principles) 


\begin{tabular}{l|l|l|l}
\hline 9 & 10 & 11 & 12 \\
\hline$(a) P W S$ & $(a) N W S$ & $(a) P W I$ & $(a) N W I$ \\
$\mathcal{W}_{c} D \rightarrow \mathcal{B}_{c} \mathcal{W}_{c} D$ & $\neg \mathcal{W}_{c} D \rightarrow \mathcal{B}_{c} \neg \mathcal{W}_{c} D$ & $\mathcal{B}_{c} \mathcal{W}_{c} D \rightarrow \mathcal{W}_{c} D$ & $\mathcal{B}_{c} \neg \mathcal{W}_{c} D \rightarrow \neg \mathcal{W}_{c} D$ \\
\hline$(b) N A S$ & $(b) P A S$ & $(b) N A I$ & $(b) P A I$ \\
$\neg \mathcal{A}_{c} D \rightarrow \mathcal{B}_{c} \neg \mathcal{A}_{c} D$ & $\mathcal{A}_{c} D \rightarrow \mathcal{B}_{c} \mathcal{A}_{c} D$ & $\mathcal{B}_{c} \neg \mathcal{A}_{c} D \rightarrow \neg \mathcal{A}_{c} D$ & $\mathcal{B}_{c} \mathcal{A}_{c} D \rightarrow \mathcal{A}_{c} D$ \\
\hline$(c) P A C$ & $(c) P W C$ & $(c) P A M$ & $(c) N A M$ \\
$\mathcal{C}_{c} \mathcal{A}_{c} D \rightarrow \mathcal{A}_{c} D$ & $\mathcal{C}_{c} \mathcal{W}_{c} D \rightarrow \mathcal{W}_{c} D$ & $\mathcal{A}_{c} D \rightarrow \mathcal{C}_{c} \mathcal{A}_{c} D$ & $\neg \mathcal{A}_{c} D \rightarrow \mathcal{C}_{c} \neg \mathcal{A}_{c} D$ \\
\hline$(d) N W C$ & $(d) N A C$ & $(d) N W M$ & $(d) P W M$ \\
$\mathcal{C}_{c} \neg \mathcal{W}_{c} D \rightarrow \neg \mathcal{W}_{c} D$ & $\mathcal{C}_{c} \neg \mathcal{A}_{c} D \rightarrow \neg \mathcal{A}_{c} D$ & $\neg \mathcal{W}_{c} D \rightarrow \mathcal{C}_{c} \neg \mathcal{W}_{c} D$ & $\mathcal{W}_{c} D \rightarrow \mathcal{C}_{c} \mathcal{W}_{c} D$ \\
\hline
\end{tabular}

Table 35 (Some boulesic-doxastic principles)

\begin{tabular}{l|l|l|l}
\hline 13 & 14 & 15 & 16 \\
\hline$(a) P B L$ & $(a) N B L$ & $(a) P B G$ & $(a) N B G$ \\
$\mathcal{B}_{c} D \rightarrow \mathcal{W}_{c} \mathcal{B}_{c} D$ & $\neg \mathcal{B}_{c} D \rightarrow \mathcal{W}_{c} \neg \mathcal{B}_{c} D$ & $\mathcal{W}_{c} \mathcal{B}_{c} D \rightarrow \mathcal{B}_{c} D$ & $\mathcal{W}_{c} \neg \mathcal{B}_{c} D \rightarrow \neg \mathcal{B}_{c} D$ \\
\hline$(b) N M L$ & $(b) P M L$ & $(b) N M G$ & $(b) P M G$ \\
$\neg \mathcal{C}_{c} D \rightarrow \mathcal{W}_{c} \neg \mathcal{C}_{c} D$ & $\mathcal{C}_{c} D \rightarrow \mathcal{W}_{c} \mathcal{C}_{c} D$ & $\mathcal{W}_{c} \neg \mathcal{C}_{c} D \rightarrow \neg \mathcal{C}_{c} D$ & $\mathcal{W}_{c} \mathcal{C}_{c} D \rightarrow \mathcal{C}_{c} D$ \\
\hline$(c) P M P$ & $(c) P B P$ & $(c) P M A$ & $(c) N M A$ \\
$\mathcal{A}_{c} \mathcal{C}_{c} D \rightarrow \mathcal{C}_{c} D$ & $\mathcal{A}_{c} \mathcal{B}_{c} D \rightarrow \mathcal{B}_{c} D$ & $\mathcal{C}_{c} D \rightarrow \mathcal{A}_{c} \mathcal{C}_{c} D$ & $\neg \mathcal{C}_{c} D \rightarrow \mathcal{A}_{c} \neg \mathcal{C}_{c} D$ \\
\hline$(d) N B P$ & $(d) N M P$ & $(d) N B A$ & $(d) P B A$ \\
$\mathcal{A}_{c} \neg \mathcal{B}_{c} D \rightarrow \neg \mathcal{B}_{c} D$ & $\mathcal{A}_{c} \neg \mathcal{C}_{c} D \rightarrow \neg \mathcal{C}_{c} D$ & $\neg \mathcal{B}_{c} D \rightarrow \mathcal{A}_{c} \neg \mathcal{B}_{c} D$ & $\mathcal{B}_{c} D \rightarrow \mathcal{A}_{c} \mathcal{B}_{c} D$ \\
\hline
\end{tabular}

Table 36 (Some boulesic-doxastic principles)

Consider the principles in tables 33-36. The first $P$ and the first $N$ stand for 'positive' and 'negative', respectively. $B, M, W$ and $A$ in the second position stand for 'belief', 'imagination', 'want' and 'acceptance', respectively. $S, C, I, M, L$, $P, G, A$ in the third position stand for 'awareness' (introspection), (access-) 'consciousness', 'infallibility', 'imaginability', 'lovability', 'approvability', 'governability' and 'acceptability', respectively. So, $P B S$, for example, stands for 'the principle of positive belief awareness', NAI for 'the principle of negative acceptance infallibility', and so on.

'1: $d 4 .(U R:(M \mathcal{B} . F T R))$ ' means that every principle in 1 (in Table 33) is provable in every system that includes $d 4$, and $U R$ or $M \mathcal{B}$ and $F T R$, given that $c$ is perfectly rational, and so on. Hence, we can establish the following results:

1: d4. (UR: (MB.FTR )). 2: d5. (UR: (MB.FTR)). 3: d5.(UR:(MB.FTR $))$. 4: (dD.d4). (UR:(MB.FTR $))$.

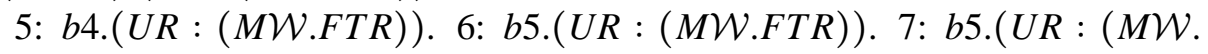
FTR )). 8: (bD.b4).(UR:(MW.FTR $))$.

9: db4.(UR:(MB.FTR)). 10: db5.(UR: (MB.FTR)). 11: (db5.dD).(UR: $(M \mathcal{B} . F T R)) .12:(d b 4 . d D) .(U R:(M \mathcal{B} . F T R))$. 


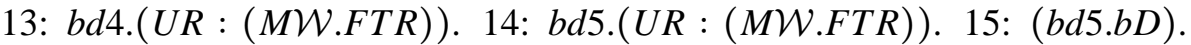
$(U R:(M \mathcal{W} . F T R)) .16:(b d 4 . b D) .(U R:(M \mathcal{W} . F T R))$.

We can employ the principles in tables 33-36 to define 64 different predicates that can be used to describe various individuals. For every principle $P$, we define a corresponding predicate $P x$ in the following way: $\Pi x(P x \leftrightarrow \Pi Y(P[Y / D, x / c]))$, where $P[Y / D, x / c]$ is like $P$ except that every occurrence of $D$ has been replaced by an occurrence of $Y$ and every occurrence of $c$ has been replaced by an occurrence of $x$. For example, from $P B S$ we define the predicate $P B S x$ in the following way: $\Pi x\left(P B S x \leftrightarrow \Pi Y\left(\mathcal{B}_{x} Y \rightarrow \mathcal{B}_{x} \mathcal{B}_{x} Y\right)\right)$, which reads as ' $x$ is (perfectly) positively belief aware iff for every $Y$, if $x$ believes $Y$, then $x$ believes that $x$ believes $Y$ '. In other words, $x$ is (perfectly) positively belief aware iff she is aware of all her beliefs. Furthermore, let $P Y Z x$ be one of the 32 predicates defined in this way that begins with $P$, and let $N Y Z x$ be one of the 32 predicates defined in this way that begins with $N$. Then we can define 32 new predicates in the following way: $\Pi x(Y Z x \leftrightarrow(P Y Z x \wedge N Y Z x))$. For example, we define the predicate $B S x$ in terms of $P B S x$ and NBS $x$ in the following way: $\Pi x(B S x \leftrightarrow(P B S x \wedge N B S x))$, which reads as ' $x$ is (perfectly) belief aware iff $x$ is both positively and negatively belief aware'. In other words, $x$ is (perfectly) belief aware iff she is aware of all her beliefs and non-beliefs. Among these predicates, eight are of the form $B Z x$, eight of the form $W Z x$, eight of the form $M Z x$ and eight of the form $A Z x$, where $Z$ stands for $S, C, I, M, A, G, L$ or $P$. Let $Z$ be interpreted in this way. Then, we can define eight new predicates in the following way: $\Pi x(Z x \leftrightarrow(B Z x \wedge W Z x \wedge M Z x \wedge A Z x))$. For example, $\Pi x(S x \leftrightarrow(B S x \wedge W S x \wedge M S x \wedge A S x))$. The informal interpretation of these predicates is as follows: $S x: x$ is perfectly (self-)aware; in other words, someone is perfectly (self-) aware iff she is aware of everything she believes and does not believe, everything she wants and does not want, everything that is imaginable or conceivable to her and everything that is not, and everything that is acceptable to her and everything that is not; $C x: x$ is perfectly (self-)(access-)conscious; $I x: x$ is perfectly (self-)infallible; $M x: x$ is perfectly (self-)imaginative; $A x: x$ is perfectly (self-)accepting; $G x: x$ is perfectly (self-)governing (autonomous); $L x: x$ is perfectly (self-)loving; $P x: x$ is perfectly (self-)approving. Finally, we will introduce one more predicate that is defined in terms of these eight basic predicates in the following way: $\Pi x(E x \leftrightarrow(S x \wedge C x \wedge I x \wedge M x \wedge A x \wedge G x \wedge L x \wedge P x))$, which says that $x$ is (perfectly) enlightened iff $x$ is perfectly (self-)aware, and so on. 


\begin{tabular}{|c|c|}
\hline Theorem & Systems \\
\hline$\Pi x(R x \rightarrow(P B S x \wedge N M S x \wedge P M C x \wedge N B C x))$ & $d 4 .(U R:(M \mathcal{B} . F T R))$ \\
\hline$\Pi x(R x \rightarrow(N B S x \wedge P M S x \wedge P B C x \wedge N M C x))$ & d5. $(U R:(M \mathcal{B} . F T R))$ \\
\hline$\Pi x(R x \rightarrow(P B I x \wedge N M I x \wedge P M M x \wedge N B M x))$ & d5. $(U R:(M \mathcal{B} . F T R))$ \\
\hline$\Pi x(R x \rightarrow(N B I x \wedge P M I x \wedge N M M x \wedge P B M x))$ & $(d D . d 4) .(U R:(M \mathcal{B} . F T R))$ \\
\hline$\Pi x(R x \rightarrow(P W L x \wedge N A L x \wedge P A P x \wedge N W P x))$ & b4. $(U R:(M \mathcal{W} . F T R))$ \\
\hline$\Pi x(R x \rightarrow(N W L x \wedge P A L x \wedge P W P x \wedge N A P x))$ & b5. $(U R:(M \mathcal{W} . F T R))$ \\
\hline$\Pi x(R x \rightarrow(P W G x \wedge N A G x \wedge P A A x \wedge N W A x))$ & b5. $(U R:(M \mathcal{W} . F T R))$ \\
\hline$\Pi x(R x \rightarrow(N W G x \wedge P A G x \wedge N A A x \wedge P W A x))$ & $(b D . b 4) \cdot(U R:(M \mathcal{W} . F T R))$ \\
\hline$\Pi x(R x \rightarrow(P W S x \wedge N A S x \wedge P A C x \wedge N W C x))$ & db4. $(U R:(M \mathcal{B} . F T R))$ \\
\hline$\Pi x(R x \rightarrow(N W S x \wedge P A S x \wedge P W C x \wedge N A C x))$ & db5. $(U R:(M \mathcal{B} . F T R))$ \\
\hline$\Pi x(R x \rightarrow(P W I x \wedge N A I x \wedge P A M x \wedge N W M x))$ & $(d b 5 . d D) .(U R:(M \mathcal{B} . F T R))$ \\
\hline$\Pi x(R x \rightarrow(N W I x \wedge P A I x \wedge N A M x \wedge P W M x))$ & $(d b 4 . d D) .(U R:(M \mathcal{B} . F T R))$ \\
\hline$\Pi x(R x \rightarrow(P B L x \wedge N M L x \wedge P M P x \wedge N B P x))$ & $b d 4 .(U R:(M \mathcal{W} . F T R))$ \\
\hline$\Pi x(R x \rightarrow(N B L x \wedge P M L x \wedge P B P x \wedge N M P x))$ & $b d 5 .(U R:(M \mathcal{W} . F T R))$ \\
\hline$\Pi x(R x \rightarrow(P B G x \wedge N M G x \wedge P M A x \wedge N B A x))$ & $(b d 5 . b D) .(U R:(M \mathcal{W} . F T R))$ \\
\hline$\Pi x(R x \rightarrow(N B G x \wedge P M G x \wedge N M A x \wedge P B A x))$ & $(b d 4 . b D) \cdot(U R:(M \mathcal{W} \cdot F T R))$ \\
\hline
\end{tabular}

Consider Table 37. We see, for example, that $\Pi x(R x \rightarrow(P B S x \wedge N M S x \wedge$ $P M C x \wedge N B C x)$ ) is provable in every system that includes $d 4$, and $U R$ or $M \mathcal{B}$ and $F T R$. In other words, in every system that contains $d 4$, and $U R$ or $M \mathcal{B}$ and $F T R$, we can prove that every perfectly rational individual is (perfectly) positively belief aware, and so on; that is, if $x$ is (perfectly) rational then for every $Y$ if $x$ believes $Y$ then $x$ believes that $x$ believes $Y$, and so on. The other results are interpreted similarly.

\begin{tabular}{l|l}
\hline Theorem & Systems \\
\hline$\Pi x(R x \rightarrow(B S x \wedge M S x \wedge M C x \wedge B C x))$ & $(d 4 . d 5)(U R:(M \mathcal{B} . F T R))$ \\
$\Pi x(R x \rightarrow(B I x \wedge M I x \wedge M M x \wedge B M x))$ & $(d D . d 4 . d 5) .(U R:(M \mathcal{B} . F T R))$ \\
$\Pi x(R x \rightarrow(W L x \wedge A L x \wedge A P x \wedge W P x))$ & $(b 4 . b 5) .(U R:(M \mathcal{W} . F T R))$ \\
$\Pi x(R x \rightarrow(W G x \wedge A G x \wedge A A x \wedge W A x))$ & $(b D . b 4 . b 5) .(U R:(M \mathcal{W} . F T R))$ \\
$\Pi x(R x \rightarrow(W S x \wedge A S x \wedge A C x \wedge W C x))$ & $(d b 4 . d b 5) .(U R:(M \mathcal{B} . F T R))$ \\
$\Pi x(R x \rightarrow(W I x \wedge A I x \wedge A M x \wedge W M x))$ & $(d D . d b 4 . d b 5) .(U R:(M \mathcal{B} . F T R))$ \\
$\Pi x(R x \rightarrow(B L x \wedge M L x \wedge M P x \wedge B P x))$ & $(b d 4 . b d 5) .(U R:(M \mathcal{W} . F T R))$ \\
$\Pi x(R x \rightarrow(B G x \wedge M G x \wedge M A x \wedge B A x))$ & $(b D . b d 4 . b d 5) .(U R:(M \mathcal{W} . F T R))$ \\
\hline \multicolumn{2}{c}{ Table 38(Some theorems in some systems) }
\end{tabular}




\begin{tabular}{|c|c|}
\hline Theorem & Systems \\
\hline$\Pi x(R x \rightarrow S x)$ & $(d 4 . d 5 . d b 4 . d b 5) .(U R:(M \mathcal{B} . F T R))$ \\
\hline$\Pi x(R x \rightarrow C x)$ & $(d 4 . d 5 . d b 4 . d b 5) .(U R:(M \mathcal{B} . F T R))$ \\
\hline$\Pi x(R x \rightarrow I x)$ & $(d D . d 4 . d 5 . d b 4 . d b 5) \cdot(U R:(M \mathcal{B} . F T R))$ \\
\hline$\Pi x(R x \rightarrow M x)$ & $(d D . d 4 . d 5 . d b 4 . d b 5) .(U R:(M \mathcal{B} . F T R))$ \\
\hline$\Pi x(R x \rightarrow A x)$ & $(b D . b 4 . b 5 . b d 4 . b d 5) \cdot(U R:(M \mathcal{W} \cdot F T R))$ \\
\hline$\Pi x(R x \rightarrow G x)$ & $(b D \cdot b 4 . b 5 . b d 4 . b d 5) \cdot(U R:(M \mathcal{W} \cdot F T R))$ \\
\hline$\Pi x(R x \rightarrow L x)$ & $(b D . b 4 . b 5 . b d 4 . b d 5) \cdot(U R:(M \mathcal{W} \cdot F T R))$ \\
\hline$\Pi x(R x \rightarrow P x)$ & $(b 4 . b 5 . b d 4 . b d 5) \cdot(U R:(M \mathcal{W} . F T R))$ \\
\hline$\Pi x(R x \rightarrow E x)$ & $(b D . b 4 . b 5 . b d 4 . b d 5 . d D \cdot d 4 . d 5 . d b 4 . d b 5) .(U R:(M \mathcal{W} \cdot M \mathcal{B} . F T R))$ \\
\hline
\end{tabular}

\begin{tabular}{l|l}
\hline Theorem & Systems \\
\hline$\Pi X \square X \leftrightarrow \square \Pi X X ; \Pi X U X \leftrightarrow U \Pi X X$ & Every \\
$\Sigma X \diamond X \leftrightarrow \diamond \Sigma X X ; \Sigma X M X \leftrightarrow M \Sigma X X$ & Every \\
$\diamond \Pi X X \rightarrow \Pi X \diamond X ; M \Pi X X \rightarrow \Pi X M X$ & Every \\
$\Sigma X \square X \rightarrow \square \Sigma X X ; \Sigma X U X \rightarrow U \Sigma X X$ & Every \\
$\Pi x\left(R x \rightarrow\left(\Pi Y \mathcal{W}_{x} Y \leftrightarrow \mathcal{W}_{x} \Pi Y Y\right)\right) ; \Pi x\left(R x \rightarrow\left(\Pi Y \mathcal{B}_{x} Y \leftrightarrow \mathcal{B}_{x} \Pi Y Y\right)\right)$ & Every \\
$\Pi x\left(R x \rightarrow\left(\Sigma Y \mathcal{A}_{x} Y \leftrightarrow \mathcal{A}_{x} \Sigma Y Y\right)\right) ; \Pi x\left(R x \rightarrow\left(\Sigma Y \mathcal{C}_{x} Y \leftrightarrow \mathcal{C}_{x} \Sigma Y Y\right)\right)$ & Every \\
$\Pi x\left(R x \rightarrow\left(\mathcal{A}_{x} \Pi Y Y \rightarrow \Pi Y \mathcal{A}_{x} Y\right)\right) ; \Pi x\left(R x \rightarrow\left(\mathcal{C}_{x} \Pi Y Y \rightarrow \Pi Y \mathcal{C}_{x} Y\right)\right)$ & Every \\
$\Pi x\left(R x \rightarrow\left(\Sigma Y \mathcal{W}_{x} Y \rightarrow \mathcal{W}_{x} \Sigma Y Y\right)\right) ; \Pi x\left(R x \rightarrow\left(\Sigma Y \mathcal{B}_{x} Y \rightarrow \mathcal{B}_{x} \Sigma Y Y\right)\right)$ & Every \\
\hline
\end{tabular}

Table 40 (Barcan-like theorems involving propositional quantifiers)

\section{Soundness and completeness theorems}

In this section, I will show that every (non-augmented) system in this paper is sound and complete with respect to its semantics (from now on, 'system' means 'non-augmented system'). The definitions of 'soundness' and 'completeness' are standard (see the introduction for some references).

Lemma 6. (Locality): Let $\mathcal{M}_{1}=\left\langle D, W, \mathfrak{R}, \mathfrak{A}, \mathfrak{D}, v_{1}\right\rangle$ and $\mathcal{M}_{2}=\left\langle D, W, \mathfrak{R}, \mathfrak{A}, \mathfrak{D}, v_{2}\right\rangle$ be two models. Since the domain of $\mathcal{M}_{1}$ is identical with the domain of $\mathcal{M}_{2}$ : $\mathcal{L}\left(\mathcal{M}_{1}\right)=\mathcal{L}\left(\mathcal{M}_{2}\right)$. We will call this language $\mathcal{L}$. Moreover, let $A$ be any closed formula of $\mathcal{L}$ such that $v_{1}$ and $v_{2}$ agree on the denotations of all the predicates, constants and matrices in it. Then for all $\omega \in W: v_{1 \omega}(A)=v_{2 \omega}(A)$.

Proof. Atomic formulas. $v_{1 \omega}\left(P a_{1} \ldots a_{n}\right)=1$ iff $\left\langle v_{1}\left(a_{1}\right), \ldots, v_{1}\left(a_{n}\right)\right\rangle \in v_{1 \omega}(P)$ iff $\left\langle v_{2}\left(a_{1}\right), \ldots, v_{2}\left(a_{n}\right)\right\rangle \in v_{2 \omega \tau}(P)$ iff $v_{2 \omega}\left(P a_{1} \ldots a_{n}\right)=1$.

Suppose that $v_{1 \omega}\left(R a_{m}\right)=0$, that $M$ is a matrix where $x_{m}$ is the first free variable in $M$ and that $a_{m}$ is the constant in $M\left[a_{1}, \ldots, a_{n} / \vec{x}\right]$ that replaces $x_{m}$. Then: 
$v_{2 \omega}\left(R a_{m}\right)=0$ and $v_{1 \omega}\left(M\left[a_{1}, \ldots, a_{n} / \vec{x}\right]\right)=1$ iff $\left\langle v_{1}\left(a_{1}\right), \ldots, v_{1}\left(a_{n}\right)\right\rangle \in v_{1 \omega}(M)$ iff $\left\langle v_{2}\left(a_{1}\right), \ldots, v_{2}\left(a_{n}\right)\right\rangle \in v_{2 \omega}(M)$ iff $v_{2 \omega}\left(M\left[a_{1}, \ldots, a_{n} / \vec{x}\right]\right)=1$.

Truth-functional connectives. Straightforward.

(口). $v_{1 \omega}(\square B)=1$ iff for all $\omega^{\prime}$ such that $\Re \omega \omega^{\prime}, v_{1 \omega^{\prime}}(B)=1$ iff for all $\omega^{\prime}$ such that $\Re \omega \omega^{\prime}, v_{2 \omega^{\prime}}(B)=1$ [the IH (the induction hypothesis)] iff $v_{2 \omega}(\square B)=1$.

Other alethic operators. Similar.

$\left(\mathcal{B}_{c} C\right) . A$ is of the form $\mathcal{B}_{c} C$. Assume that $v_{1 \omega}\left(\mathcal{B}_{c} C\right)=1$. We have two cases: $v_{1 \omega}(R c)=0$ or $v_{1 \omega}(R c)=1$. Suppose $v_{1 \omega}(R c)=0$. Then $v_{2 \omega}(R c)=0$. Hence, $v_{2 \omega}\left(\mathcal{B}_{c} C\right)=1$. And vice versa. Suppose $v_{1 \omega}(R c)=1$. Then for all $\omega^{\prime}$ such that $\mathfrak{D} v_{1}(c) \omega \omega^{\prime}: v_{1 \omega^{\prime}}(C)=1$. Accordingly, for all $\omega^{\prime}$ such that $\mathfrak{D} v_{2}(c) \omega \omega^{\prime}: v_{2 \omega^{\prime}}(C)=$ 1 [by assumption and the IH]. Furthermore, $v_{2 \omega}(R c)=1$. Hence, $v_{2 \omega}\left(\mathcal{B}_{c} C\right)=1$. And vice versa. Consequently, $v_{1 \omega}\left(\mathcal{B}_{c} C\right)=1$ iff $v_{2 \omega}\left(\mathcal{B}_{c} C\right)=1$.

Other boulesic and doxastic operators. Similar.

(П). $v_{1 \omega}(\Pi x B)=1$ iff for all $k_{d} \in \mathcal{L}, v_{1 \omega}\left(B\left[k_{d} / x\right]\right)=1$ iff for all $k_{d} \epsilon$ $\mathcal{L}, v_{2 \omega}\left(B\left[k_{d} / x\right]\right)=1$ [by the $\mathrm{IH}$, and the fact that $v_{1 \omega}\left(k_{d}\right)=v_{2 \omega}\left(k_{d}\right)=d$ ] iff $v_{2 \omega}(\Pi x B)=1$.

The particular quantifier. Similar.

Lemma 7. (Denotation): Let $\mathcal{M}=\langle D, W, \mathfrak{R}, \mathfrak{A}, \mathfrak{D}, v\rangle$ be a model and let $A$ be any formula of $\mathcal{L}(\mathcal{M})$ with at most one free variable $x$. Furthermore, let $a$ and $b$ be any two constants such that $v(a)=v(b)$. Then, $v_{\omega}(A[a / x])=v_{\omega}(A[b / x])$, for any $\omega \in W$.

Proof. Atomic formulas. Suppose that the formula has one occurrence of ' $a$ ' distinct from each $a_{i}$. Then, $v_{\omega}\left(P a_{1} \ldots a \ldots a_{n}\right)=1$ iff $\left\langle v\left(a_{1}\right), \ldots, v(a), \ldots, v\left(a_{n}\right)\right\rangle \epsilon$ $v_{\omega}(P)$ iff $\left\langle v\left(a_{1}\right), \ldots, v(b), \ldots, v\left(a_{n}\right)\right\rangle \in v_{\omega}(P)$ iff $v_{\omega}\left(P a_{1} \ldots b \ldots a_{n}\right)=1$. Other cases. Similar.

Suppose $v_{\omega}\left(R a_{m}\right)=0$, that $M$ is a matrix where $x_{m}$ is the first free variable in $M$ and that $a_{m}$ is the constant in $M\left[a_{1}, \ldots, a, \ldots, a_{n} / \vec{x}\right]\left(M\left[a_{1}, \ldots, b, \ldots, a_{n} / \vec{x}\right]\right)$ that replaces $x_{m}$. (To illustrate, we assume that the formula has one occurrence of ' $a$ ' distinct from each $a_{i}$ and that $a_{m}$ is not $a(b)$.) Then: $v_{\omega}\left(M\left[a_{1}, \ldots, a, \ldots, a_{n} / \vec{x}\right]\right)=$ 1 iff $\left\langle v\left(a_{1}\right), \ldots, v(a), \ldots, v\left(a_{n}\right)\right\rangle \in v_{\omega}(M)$ iff $\left\langle v\left(a_{1}\right), \ldots, v(b), \ldots, v\left(a_{n}\right)\right\rangle \in v_{\omega}(M)$ iff $v_{\omega}\left(M\left[a_{1}, \ldots, b, \ldots, a_{n} \mid \vec{x}\right]\right)=1$.

Truth-functional connectives. Straightforward.

(口). $v_{\omega}(\square B[a / x])=1$ iff for all $\omega^{\prime}$ such that $\mathfrak{R} \omega \omega^{\prime}, v_{\omega^{\prime}}(B[a / x])=1$ iff for all $\omega^{\prime}$ such that $\Re \omega \omega^{\prime}, v_{\omega^{\prime}}(B[b / x])=1[$ the $\mathrm{IH}]$ iff $v_{\omega}(\square B[b / x])=1$.

Other alethic operators. Similar.

$\left(\mathcal{B}_{t}\right) . A$ is of the form $\mathcal{B}_{t} C$. Either $v_{\omega}(R t)=1$ or $v_{\omega}(R t)=0$. We have already shown that the result holds if $v_{\omega}(R t)=0$. Accordingly, suppose that $v_{\omega}(R t)=1$. Since $x$ is the only free variable, $t$ cannot be a variable distinct from $x$. So, $t$ is either 
$x$ or a constant. Suppose $t$ is $x$. Then $v_{\omega}\left(\mathcal{B}_{x} C[a / x]\right)=1$ iff $v_{\omega}\left(\mathcal{B}_{a} C[a / x]\right)=1$ iff for all $\omega^{\prime}$ such that $\mathfrak{D} v(a) \omega \omega^{\prime}, v_{\omega^{\prime}}(C[a / x])=1$ iff for all $\omega^{\prime}$ such that $\mathfrak{D} v(b) \omega \omega^{\prime}$, $v_{\omega^{\prime}}(C[b / x])=1$ [by the fact that $v(a)=v(b)$ and the IH] iff $v_{\omega}\left(\mathcal{B}_{b} C[b / x]\right)=1$ iff $v_{\omega}\left(\mathcal{B}_{x} C[b / x]\right)=1$. Suppose $t$ is a constant, say $c$. Then $v_{\omega}\left(\mathcal{B}_{c} C[a / x]\right)=1 \mathrm{iff}$ for all $\omega^{\prime}$ such that $\mathfrak{D} v(c) \omega \omega^{\prime}, v_{\omega^{\prime}}(C[a / x])=1$ iff for all $\omega^{\prime}$ such that $\mathfrak{D} v(c) \omega \omega^{\prime}$, $v_{\omega^{\prime}}(C[b / x])=1$ [by the $\left.\mathrm{IH}\right]$ iff $v_{\omega}\left(\mathcal{B}_{c} C[b / x]\right)=1$.

Other boulesic and doxastic operators. Similar.

(П). If $x=y$, the result is trivial, for then $A[a / x]=A[b / x]=A$. So, suppose that $x$ and $y$ are distinct. Then, $(\Pi y B)[b / x]=\Pi y(B[b / x])$ and $(B[b / x])[a / y]=$ $(B[a / y])[b / x] . \quad v_{\omega}((\Pi y B)[a / x])=1$ iff $v_{\omega}(\Pi y(B[a / x]))=1$ iff for all $k_{d} \epsilon$ $\mathcal{L}(\mathcal{M}), v_{\omega}\left((B[a / x])\left[k_{d} / y\right]\right)=1$ iff for all $k_{d} \in \mathcal{L}(\mathcal{M}), v_{\omega}\left(\left(B\left[k_{d} / y\right]\right)[a / x]\right)=1$ iff for all $k_{d} \in \mathcal{L}(\mathcal{M}), v_{\omega}\left(\left(B\left[k_{d} / y\right]\right)[b / x]\right)=1$ [the IH] iff for all $k_{d} \in \mathcal{L}(\mathcal{M})$, $v_{\omega}\left((B[b / x])\left[k_{d} / y\right]\right)=1$ iff $v_{\omega}(\Pi y(B[b / x]))=1$ iff $v_{\omega}((\Pi y B)[b / x])=1$.

The particular quantifier. Similar.

\subsection{Soundness theorem}

Let $\mathcal{M}=\langle D, W, \mathfrak{R}, \mathfrak{A}, \mathfrak{D}, v\rangle$ be a model and let $\mathcal{B}$ be a branch in a tableau. Then $\mathcal{B}$ is satisfiable in $\mathcal{M}$ iff there is a function $f$ from $0,1,2, \ldots$ to $W$ such that

(i) $A$ is true in $f(i)$ in $\mathcal{M}$, for every node $A, i$ on $\mathcal{B}$,

(ii) if $i r j$ is on $\mathcal{B}$, then $\mathfrak{R} f(i) f(j)$ in $\mathcal{M}$,

(iii) if $i A c j$ is on $\mathcal{B}$, then $\mathfrak{A} v(c) f(i) f(j)$ in $\mathcal{M}$.

(iv) if $i D c j$ is on $\mathcal{B}$, then $\mathfrak{D} v(c) f(i) f(j)$ in $\mathcal{M}$.

(v) if $i=j$ is on $\mathcal{B}$, then $f(i)$ is $f(j)$.

We shall say that $f$ shows that $\mathcal{B}$ is satisfiable in $\mathcal{M}$ if these conditions are fulfilled.

Lemma 8. (Soundness Lemma): Let $\mathcal{B}$ be any branch of a tableau and let $\mathcal{M}$ be any model. If $\mathcal{B}$ is satisfiable in $\mathcal{M}$ and a tableau rule is applied to it, then there is a model $\mathcal{M}^{\prime}$ and an extension of $\mathcal{B}, \mathcal{B}^{\prime}$, such that $\mathcal{B}^{\prime}$ is satisfiable in $\mathcal{M}^{\prime}$.

Proof. Let $f$ be a function that shows that the branch $\mathcal{B}$ is satisfiable in $\mathcal{M}$.

Connectives and modal operators. Straightforward.

$(\mathcal{W})$. Suppose that $R c, i, \mathcal{W}_{c} C, i$, and $i A c j$ are on $\mathcal{B}$, and that we apply the $\mathcal{W}$ rule. Then we get an extension of $\mathcal{B}$ that includes $C, j$. Since $\mathcal{B}$ is satisfiable in $\mathcal{M}$, $\mathcal{W}_{c} C$ is true in $f(i)$ and $R c$ is true in $f(i)$. Moreover, for any $i$ and $j$ such that $i A c j$ is on $\mathcal{B}, \mathfrak{A} v(c) f(i) f(j)$. Thus by the truth conditions for $\mathcal{W}_{c} C, C$ is true in $f(j)$.

$(\mathcal{C})$. Suppose that $R c, i$ and $\mathcal{C}_{c} B, i$ are on $\mathcal{B}$ and that we apply the $\mathcal{C}$-rule. Then we get an extension of $\mathcal{B}$ that includes nodes of the form $i D c j$ and $B, j$. Since $\mathcal{B}$ is satisfiable in $\mathcal{M}$, both $\mathcal{C}_{c} B$ and $R c$ are true in $f(i)$. Accordingly, for some $\omega$ in $W, \mathfrak{D} v(c) f(i) \omega$ and $B$ is true in $\omega$ [by the truth conditions for $\mathcal{C}_{c} B$ and the fact that 
$R c$ is true in $f(i)$ ]. Let $f^{\prime}$ be the same as $f$ except that $f^{\prime}(j)=\omega$. Since $f$ and $f^{\prime}$ differ only at $j, f^{\prime}$ shows that $\mathcal{B}$ is satisfiable in $\mathcal{M}$. Moreover, by definition $\mathfrak{D} v(c) f^{\prime}(i) f^{\prime}(j)$, and $B$ is true in $f^{\prime}(j)$.

Other boulesic and doxastic cases. Similar.

(П). Suppose that $\Pi x A, i$ is on $\mathcal{B}$ and that we apply the $\Pi$-rule. Then we get an extension of $\mathcal{B}$ that includes a node of the form $A[a / x], i$. Since $\mathcal{B}$ is satisfiable in $\mathcal{M}, \mathcal{M}$ makes $\Pi x A$ true in $f(i)$. Accordingly, $A\left[k_{d} / x\right]$ is true in $f(i)$ in $\mathcal{M}$, for all $k_{d} \in \mathcal{L}(\mathcal{M})$. Let $d$ be such that $v(a)=v\left(k_{d}\right)$. By the Denotation Lemma, $A[a / x]$ is true in $f(i)$ in $\mathcal{M}$. Hence, we can take $\mathcal{M}^{\prime}$ to be $\mathcal{M}$.

$(\Sigma)$. Suppose that $\Sigma x A, i$ is on $\mathcal{B}$ and that we apply the $\Sigma$-rule to get an extension of $\mathcal{B}$ that includes a node of the form $A[c / x], i$ (where $c$ is new). $\Sigma x A$ is true in $f(i)$ in $\mathcal{M}$, for $\mathcal{B}$ is satisfiable in $\mathcal{M}$. Accordingly, there is some $k_{d} \in \mathcal{L}(\mathcal{M})$ such that $\mathcal{M}$ makes $A\left[k_{d} / x\right]$ true in $f(i)$. Let $\mathcal{M}^{\prime}=\left\langle D, W, \mathfrak{R}, \mathfrak{A}, \mathfrak{D}, v^{\prime}\right\rangle$ be the same as $\mathcal{M}$ except that $v^{\prime}(c)=d$. $A\left[k_{d} / x\right]$ is true in $f(i)$ in $\mathcal{M}^{\prime}$ since $c$ does not occur in $A\left[k_{d} / x\right]$, by the Locality Lemma. By the Denotation Lemma and the fact that $v^{\prime}(c)=d=v^{\prime}\left(k_{d}\right), A[c / x]$ is true in $f(i)$ in $\mathcal{M}^{\prime}$. Furthermore, $\mathcal{M}^{\prime}$ makes all other formulas on the branch true at their respective worlds as well, by the Locality Lemma, since $c$ does not occur in any other formula on the branch.

$(\neg \Pi)$ and $(\neg \Sigma)$. Straightforward.

Accessibility rules. Here is one example.

$(T-W N I)$. Suppose that $i A c j$ and $j r k$ are on $\mathcal{B}$, and that we apply $(T-$ $W N I$ ) to give an extended branch containing $j D c k$. Since $\mathcal{B}$ is satisfiable in $\mathcal{M}$, $\mathfrak{A} v(c) f(i) f(j)$ and $\mathfrak{R} f(j) f(k)$. Hence, $\mathfrak{D} v(c) f(j) f(k)$ since $\mathcal{M}$ satisfies condition $C-W N I$. Consequently, the extension of $\mathcal{B}$ is satisfiable in $\mathcal{M}$.

Other cases. Similar.

Theorem 9. (Soundness Theorem): Every system $S$ in this paper is strongly sound with respect to its semantics.

Proof. Let $\mathbf{M}$ be the class of models that corresponds to $S$. We show the 'contraposition'. So, suppose that $B$ does not follow from $\Gamma$ in $\mathbf{M}$. Then the premises in $\Gamma$ are true and the conclusion $B$ false in some world $\omega$ in $\mathbf{M}$. Consider an $S$-tableau whose initial list consists of $A, 0$ for every $A \in \Gamma$ and $\neg B, 0$, where ' 0 ' refers to $\omega$. Then the initial list is satisfiable in $\mathbf{M}$. If we apply a rule to this list, it produces at least one extension that is satisfiable in $\mathbf{M}$ (by the Soundness Lemma). Hence, we can find a whole branch such that every initial section of this branch is satisfiable in M. Suppose this branch is closed. Then some sentence is be both true and false in some possible world in M. Still, this is impossible. Consequently, the tableau is open. In conclusion, $B$ is not derivable from $\Gamma$ in $S$. 


\subsection{Completeness theorem}

Definition 10. (Induced Model): Let $\mathcal{B}$ be an open complete branch of a tableau, let $i, j, k$, etc. be numbers on $\mathcal{B}$, and let I be the set of numbers on $\mathcal{B}$. We shall say that $i \rightleftharpoons j$ just in case $i=j$, or ' $i=j$ ' or ' $j=i$ ' occurs on $\mathcal{B}$. $\rightleftharpoons$ is an equivalence relation and $[i]$ is the equivalence class of $i$. Moreover, let $C$ be the set of all constants on $\mathcal{B}$. Define $a \sim b$ to mean that $a=b, 0$ is on the branch. $a \sim b$ is obviously an equivalence relation. Let $[a]$ be the equivalence class of $a$ under $\sim$. The model $\mathcal{M}=\langle D, W, \mathfrak{R}, \mathfrak{A}, \mathfrak{D}, v\rangle$ induced by $\mathcal{B}$ is defined as follows. $D=\{[a]: a \in C\}$ (or, if $C=\varnothing, D=\{o\}$ for an arbitrary $o$ ). (o is not in the extension of anything.) $W=\left\{\omega_{[i]}\right.$ : i occurs on $\left.\mathcal{B}\right\}, \mathfrak{R} \omega_{[i]} \omega_{[j]}$ iff irj occurs on $\mathcal{B}, \mathfrak{A} v(a) \omega_{[i]} \omega_{[j]}$ iff iAaj and $\mathfrak{D} v(a) \omega_{[i]} \omega_{[j]}$ iff iDaj occurs on $\mathcal{B} . v(a)=[a]$, and $\left\langle\left[a_{1}\right], \ldots,\left[a_{n}\right]\right\rangle \in v_{\omega_{[i]}}(P)$ iff $P a_{1} \ldots a_{n}, i$ is on $\mathcal{B}$, given that $P$ is any n-place predicate other than identity. If $\neg R a_{m}, i$ occurs on $\mathcal{B}$ and $M$ is an n-place matrix with instantiations on the branch (where $x_{m}$ is the first free variable in $M$ and $a_{m}$ is the constant in $M\left[a_{1}, \ldots, a_{n} / \vec{x}\right]$ that replaces $\left.x_{m}\right)$, then $\left\langle\left[a_{1}\right], \ldots,\left[a_{n}\right]\right\rangle \in v_{\omega_{[i]}}(M)$ iff $M\left[a_{1}, \ldots, a_{n} / \vec{x}\right]$, i occurs on $\mathcal{B}$. (Due to the identity rules this is well defined.) When we have $a=b, 0, b=c, 0$, etc. we choose one single object for all constants to denote.

If a tableau system does not include $T-d O$ or $T-\mathcal{W} O, \rightleftharpoons$ is reduced to identity and $[i]=\{i\}$. Hence, in such systems, we may take $W$ to be $\left\{\omega_{i}:\right.$ i occurs on $\left.\mathcal{B}\right\}$ and dispense with the equivalence classes.

Lemma 11. (Completeness Lemma): Let $\mathcal{B}$ be an open branch in a complete tableau and let $\mathcal{M}$ be a model induced by $\mathcal{B}$. Then, for every formula $A$ :

(i) if $A$, $i$ is on $\mathcal{B}$, then $v_{\omega_{[i]}}(A)=1$, and

(ii) if $\neg A$, $i$ is on $\mathcal{B}$, then $v_{\omega_{[i]}}(A)=0$.

Proof. Atomic formulas. $P a_{1} \ldots a_{n}, i$ is on $\mathcal{B} \Rightarrow\left\langle\left[a_{1}\right], \ldots,\left[a_{n}\right]\right\rangle \in v_{\omega_{[i]}}(P) \Rightarrow$ $\left\langle v\left(a_{1}\right), \ldots, v\left(a_{n}\right)\right\rangle \in v_{\omega_{[i]}}(P) \Rightarrow v_{\omega_{[i]}}\left(P a_{1} \ldots a_{n}\right)=1$.

$\neg P a_{1} \ldots a_{n}, i$ is on $\mathcal{B} \Rightarrow P a_{1} \ldots a_{n}, i$ is not on $\mathcal{B}(\mathcal{B}$ open $) \Rightarrow\left\langle\left[a_{1}\right], \ldots,\left[a_{n}\right]\right\rangle \notin$ $v_{\omega_{[i]}}(P) \Rightarrow\left\langle v\left(a_{1}\right), \ldots, v\left(a_{n}\right)\right\rangle \notin v_{\omega_{[i]}}(P) \Rightarrow v_{\omega_{[i]}}\left(P a_{1} \ldots a_{n}\right)=0$.

Suppose that $M$ is a matrix where $x_{m}$ is the first free variable and $a_{m}$ is the constant in $M\left[a_{1}, \ldots, a_{n} / \vec{x}\right]$ that replaces $x_{m}$ and that $v_{\omega_{[i]}}\left(R a_{m}\right)=0$. Then: $M\left[a_{1}, \ldots, a_{n} / \vec{x}\right], i$ occurs on $\mathcal{B} \Rightarrow\left\langle\left[a_{1}\right], \ldots,\left[a_{n}\right]\right\rangle \in v_{\omega_{[i]}}(M) \Rightarrow\left\langle v\left(a_{1}\right), \ldots, v\left(a_{n}\right)\right\rangle$ $\in v_{\omega_{[i]}}(M) \Rightarrow v_{\omega_{[i]}}\left(M\left[a_{1}, \ldots, a_{n} / \vec{x}\right]\right)=1$. Furthermore, $\neg M\left[a_{1}, \ldots, a_{n} / \vec{x}\right], i$ occurs on $\mathcal{B} \Rightarrow M\left[a_{1}, \ldots, a_{n} / \vec{x}\right], i$ is not on $\mathcal{B}(\mathcal{B}$ open $) \Rightarrow\left\langle\left[a_{1}\right], \ldots,\left[a_{n}\right]\right\rangle \notin v_{\omega_{[i]}}(M)$ $\Rightarrow\left\langle v\left(a_{1}\right), \ldots, v\left(a_{n}\right)\right\rangle \notin v_{\omega_{[i]}}(M) \Rightarrow v_{\omega_{[i]}}\left(M\left[a_{1}, \ldots, a_{n} / \vec{x}\right]\right)=0$. 
1.

$a=b, i$ is on $\mathcal{B} \Rightarrow a \sim b(T-N=) \Rightarrow[a]=[b] \Rightarrow v(a)=v(b) \Rightarrow v_{\omega_{[i]}}(a=b)=$

$\neg a=b, i$ is on $\mathcal{B} \Rightarrow a=b, 0$ is not on $\mathcal{B}(\mathcal{B}$ open $) \Rightarrow$ it is not the case that $a \sim b$ $\Rightarrow[a] \neq[b] \Rightarrow v(a) \neq v(b) \Rightarrow v_{\omega_{[i]}}(a=b)=0$.

Other truth-functional connectives and modal operators. Straightforward.

Boulesic and doxastic operators. $(\neg \mathcal{B})$. Suppose $\neg \mathcal{B}_{c} D, i$ is on $\mathcal{B}$. Furthermore, suppose that $R c, i$ is not on $\mathcal{B}$. Then $\neg R c, i$ is on $\mathcal{B}$ [by $C U T$ ]. Hence, $\mathcal{B}_{c} D$ is false in $\omega_{[i]}$ by definition and previous steps. Suppose $R c, i$ is on $\mathcal{B}$. Then the $\neg \mathcal{B}$-rule has been applied to $\neg \mathcal{B}_{c} D, i$ and we have $\mathcal{C}_{c} \neg D, i$ on $\mathcal{B}$. For the branch is complete. Then the $\mathcal{C}$-rule has been applied to $\mathcal{C}_{c} \neg D, i$, since the branch is complete. Hence, for some new $j, i D c j$ and $\neg D, j$ occur on $\mathcal{B}$. By the induction hypothesis, $\mathfrak{D} v(c) \omega_{[i]} \omega_{[j]}$, and $D$ is false in $\omega_{[j]}$. Since $R c, i$ is on $\mathcal{B}, v(c)$ is perfectly rational in $\omega_{[i]}$. Consequently, $\mathcal{B}_{c} D$ is false in $\omega_{[i]}$, as required.

Other boulesic and doxastic operators. Similar.

Quantifiers. $(\Sigma)$. Suppose that $\Sigma x D, i$ is on the branch. The tableau is complete; so, $(\Sigma)$ has been applied. Hence, for some $c, D[c / x], i$ is on the branch. Accordingly, $v_{\omega_{[i]}}(D[c / x])=1$, by $(\mathrm{IH})$. For some $k_{d} \in \mathcal{L}(\mathcal{M}), v(c)=d$, and $v\left(k_{d}\right)=d$. Therefore, $v_{\omega_{[i]}}\left(D\left[k_{d} / x\right]\right)=1$, by the Denotation Lemma. In conclusion, $v_{\omega_{[i]}}(\Sigma x D)=1$. Suppose that $\neg \Sigma x D, i$ is on the branch. The tableau is complete; so, $(\neg \Sigma)$ has been applied. Hence, $\Pi x \neg D, i$ is on the branch. Again, since the tableau is complete ( $\Pi$ ) has been applied. Accordingly, for all $c \in C$, $\neg D[c / x], i$ is on the branch. It follows that $v_{\omega_{[i]}}(D[c / x])=0$ for all $c \in C$ [by the induction hypothesis]. If $k_{d} \in \mathcal{L}(\mathcal{M})$, then for some $c \in C, v(c)=v\left(k_{d}\right)$. By the Denotation Lemma, for all $k_{d} \in \mathcal{L}(\mathcal{M}), v_{\omega_{[i]}}\left(D\left[k_{d} / x\right]\right)=0$. In conclusion, $v_{\omega_{[i]}}(\Sigma x D)=0$.

The case for $\Pi$ is similar.

Theorem 12. (Completeness Theorem): Every system in this paper is strongly complete with respect to its semantics.

Proof. First we establish that the theorem holds for the weakest system $\mathcal{B D}$. Then we show how it can be extended to stronger systems. Let $\mathbf{M}$ be the class of models that corresponds to $\mathcal{B D}$.

We prove the 'contraposition'. Suppose that $B$ is not derivable from $\Gamma$ in $\mathcal{B D}$. Then it is not the case that there is a closed $\mathcal{B D}$-tableau whose initial list comprises $A, 0$ for every $A$ in $\Gamma$ and $\neg B, 0$. Let $t$ be a complete $\mathcal{B D}$-tableau whose initial list comprises $A, 0$ for every $A$ in $\Gamma$ and $\neg B, 0$. Then $t$ must be open. Accordingly, there is at least one open branch in $t$, say $\mathcal{B}$. The model induced by $\mathcal{B}$ makes all the premises in $\Gamma$ true and $B$ false in $\omega_{[0]}$. Consequently, it is not the case that $B$ follows from $\Gamma$ in $\mathbf{M}$. 
To show that all extensions of $\mathcal{B D}$ are complete with respect to their semantics, we have to check that the model induced by the open branch in each case is of the right kind. To do this we first go through every single semantic condition, then we combine each of the individual arguments. Here is one step in the proof to illustrate the method.

$C-W B P$. Suppose that $\mathfrak{A} v(c) \omega_{[i]} \omega_{[j]}$. Then $i A c j$ occurs on $\mathcal{B}$ [by the definition of an induced model]. Since $\mathcal{B}$ is complete $(T-W B P)$ has been applied. Hence, for some $k, j D c k$ and $j r k$ are on $\mathcal{B}$. Accordingly, for some $\omega_{[k]}, \mathfrak{D} v(c) \omega_{[j]} \omega_{[k]}$ and $\mathfrak{R} \omega_{[j]} \omega_{[k]}$, as required [by the definition of an induced model].

Other cases. Similar.

\section{Some examples}

In this section, I will prove some examples that were mentioned in the introduction. First, I will show that the following instance of (ii) in Example 2 is a theorem in every system that includes $T-M \mathcal{B}, T-\mathcal{W} P$ and $T-a b 4$ : if $x$ is perfectly rational, then $x$ wants to pass right through this stonewall only if $x$ believes that it is possible to pass right through this stonewall. This instance of (ii) can be symbolised in the following way in our systems: $\Pi x\left(R x \rightarrow\left(\mathcal{W}_{x} P x \rightarrow \mathcal{B}_{x} \diamond P x\right)\right)$. The following tableau establishes this result:

$$
\begin{gathered}
(1) \neg \prod x\left(R x \rightarrow\left(\mathcal{W}_{x} P x \rightarrow \mathcal{B}_{x} \diamond P x\right)\right), 0 \\
(2) \Sigma x \neg\left(R x \rightarrow\left(\mathcal{W}_{x} P x \rightarrow \mathcal{B}_{x} \diamond P x\right)\right), 0[1, \neg \Pi] \\
(3) \neg\left(R c \rightarrow\left(\mathcal{W}_{c} P c \rightarrow \mathcal{B}_{c} \diamond P c\right)\right), 0[2, \Sigma] \\
(4) R c, 0[3, \neg \rightarrow] \\
(5) \neg\left(\mathcal{W}_{c} P c \rightarrow \mathcal{B}_{c} \diamond P c\right), 0[3, \neg \rightarrow] \\
(6) \mathcal{W}_{c} P c, 0[5, \neg \rightarrow] \\
(7) \neg \mathcal{B}_{c} \diamond P c, 0[5, \neg \rightarrow] \\
\text { (8) } \mathcal{C}_{c} \neg \diamond P c, 0[4,7, \neg \mathcal{B}] \\
\text { (9) } 0 D c 1[4,8, \mathcal{C}] \\
(10) \neg \diamond P c, 1[4,8, \mathcal{C}] \\
(11) \square \neg P c, 1[10, \neg \diamond] \\
(12) 0 r 1[9, T-M \mathcal{B}] \\
(13) 1 A c 2[T-\mathcal{W} P] \\
(14) 1 r 2[T-\mathcal{W} P] \\
(15) 0 A c 2[12,13, T-a b 4] \\
(16) P c, 2[4,6,15, \mathcal{W}] \\
\text { (17) } \neg P c, 2[11,14, \square] \\
(18) *[16,17]
\end{gathered}
$$


Since the system that includes $T-M \mathcal{B}, T-\mathcal{W} P$ and $T-a b 4$ is valid with respect to the class of all models that satisfy $C-M \mathcal{B}, C-\mathcal{W} P$ and $C-a b 4$ (by the soundness theorems in Section 6), it follows that $\Pi x\left(R x \rightarrow\left(\mathcal{W}_{x} P x \rightarrow \mathcal{B}_{x} \diamond P x\right)\right)$ is valid in the class of all models that satisfy these conditions. ${ }^{14}$ Q.E.D.

Second, we will turn to The Doctoral Student Argument (see Example 3). Recall that this argument includes the following premises and conclusions:

(P1) Every student in the room wants to become a doctor some time in the future. (P2) Carl is a student in the room. So, (C3) Carl wants to become a doctor some time in the future. (P4) Carl believes that it is (historically) necessary that he will become a doctor some time in the future only if he studies hard. Hence, (C5) if Carl is perfectly rational (wise), Carl wants to study hard.

This argument can be symbolised in the following way: $\left(\mathrm{P}^{\prime}\right) \Pi x\left(S x \rightarrow \mathcal{W}_{x} D x\right)$ (Every student in the room wants to become a doctor some time in the future), where ' $S x$ ' stands for ' $x$ is a student in the room' and ' $D x$ ' for ' $x$ will become a doctor some time in the future'. ( $\mathrm{P}^{\prime}$ ) $S c$ (Carl is a student in the room), where ' $c$ ' refers to Carl. $\left(\mathrm{C}^{\prime}\right) \mathcal{W}_{c} D c$ (Carl wants to become a doctor some time in the future). ( $\left.4^{\prime}\right) \mathcal{B}_{c} \square(D c \rightarrow H c)$ (Carl believes that it is (historically) necessary that he will become a doctor some time in the future only if he studies hard), where ' $H c$ ' stands for ' $c$ (Carl) studies hard'. (C5') $R c \rightarrow \mathcal{W}_{c} H c$ (If Carl is perfectly rational (wise), Carl wants to study hard). (C3') follows from $\left(\mathrm{P}^{\prime}\right)$ and $\left(\mathrm{P} 2^{\prime}\right)$ in every system in this paper. $\left(\mathrm{C}^{\prime}\right)$ follows from $\left(\mathrm{C}^{\prime}\right)$ and $\left(\mathrm{P}^{\prime}\right)$ in every system that includes the tableau rules $T-d T$ and $T-M \mathcal{W}$. Let us now show this. (' $M P$ ' is an abbreviation of Modus Ponens, which is a rule that is derivable in our systems.) (3) is the 'negation' of $\left(\mathrm{C}^{\prime}\right)$.

$$
\begin{gathered}
\text { (1) } \Pi x\left(S x \rightarrow \mathcal{W}_{x} D x\right), 0\left[\mathrm{P}^{\prime}\right] \\
\text { (2) } S c, 0\left[\mathrm{P} 2^{\prime}\right] \\
\text { (3) } \neg \mathcal{W}_{c} D c, 0\left[T h e \text { 'negation' of } \mathrm{C}^{\prime}\right] \\
\text { (4) } S c \rightarrow \mathcal{W}_{c} D c, 0[1, \Pi] \\
\text { (5) } \mathcal{W}_{c} D c, 0[2,4, M P] \\
(6) *[3,5]
\end{gathered}
$$

Now we use the conclusion $\left(\mathrm{C}^{\prime}\right)$ as a premise in a new argument for $\left(\mathrm{C}^{\prime}\right)$. (3) below is the 'negation' of $\left(\mathrm{C}^{\prime}\right)$.

\footnotetext{
${ }^{14}$ Note that $\Pi x\left(R x \rightarrow\left(\mathcal{W}_{x} P x \rightarrow \mathcal{B}_{x} \diamond P x\right)\right)$ also can be proved in many other systems in this paper, for example, in every system that includes $T-\mathcal{W} P$ and $T-d b 4$. Similar remarks apply to many other theorems that are mentioned in this paper.
} 
(1) $\mathcal{W}_{c} D c, 0\left[\mathrm{C}^{\prime}\right]$

(2) $\mathcal{B}_{c} \square(D c \rightarrow H c), 0\left[\mathrm{P}^{\prime}\right]$

(3) $\neg\left(R c \rightarrow \mathcal{W}_{c} H c\right), 0$ [The 'negation' of $\mathrm{C}^{\prime}$ ]

(4) $R c, 0[3, \neg \rightarrow]$

(5) $\neg \mathcal{W}_{c} H c, 0[3, \neg \rightarrow]$

(6) $\mathcal{A}_{c} \neg H c, 0[4,5, \neg \mathcal{W}]$

(7) $0 A c 1[4,6, \mathcal{A}]$

(8) $\neg H c, 1[4,6, \mathcal{A}]$

(9) $D c, 1[4,1,7, \mathcal{W}]$

(10) $0 D c 0[T-d T]$

(11) $\square(D c \rightarrow H c), 0[4,2,10, \mathcal{B}]$

(12) $0 r 1[7, T-M \mathcal{W}]$

(13) $D c \rightarrow H c, 1[11,12, \square]$

(14) $H c, 1[9,13, M P]$

$(15) *[8,14]$

The tableaux above show that both subarguments in The Doctoral Student Argument are 'syntactically' valid. In the first argument, we have not used any special tableau rules. Hence, the conclusion is derivable from the premises in every system in this paper. By the soundness results (see Section 6) it follows that the argument is valid in the class of all models. In the second subargument, we have used the tableau rules $T-M \mathcal{W}$ and $T-d T$. Hence, the conclusion is derivable from the premises in every system that includes these rules. By the soundness results in Section 6 it follows that the argument is valid in the class of all models that satisfy $C-M \mathcal{W}$ and $C-d T$. It follows that The Doctoral Student Argument is valid in the class of all models that satisfy $C-M \mathcal{W}$ and $C-d T$. Q.E.D.

Third, we will now show that the conclusion in The Doctoral Student Argument II (see Example 4) is not derivable from the premises in our weakest system $\mathcal{B D}$. Recall that this argument consists of the following premises and conclusion: (P1) Every student in the room wants to become a doctor some time in the future. (P2) Carl is a student in the room. (P3) Carl believes that it is (historically) necessary that he will become a doctor some time in the future only if he studies hard. Hence, (C4) Carl wants to study hard. These sentences can be symbolised in the following way: $\left(\mathrm{P}^{\prime}\right) \Pi x\left(S x \rightarrow \mathcal{W}_{x} D x\right),\left(\mathrm{P} 2^{\prime}\right) S c,\left(\mathrm{P}^{\prime}\right) \mathcal{B}_{c} \square(D x \rightarrow H x)$ and $\left(\mathrm{C}^{\prime}\right) \mathcal{W}_{c} H c$, where the predicates are interpreted as in The Doctoral Student Argument. To show this, we construct a semantic tableau that starts with the premises and the negation of the conclusion. More precisely, our tableau will begin with the following nodes: $\left(\mathrm{P} 1^{\prime}\right), 0,\left(\mathrm{P} 2^{\prime}\right), 0,\left(\mathrm{P} 3^{\prime}\right), 0$ and $\neg\left(\mathrm{C} 4^{\prime}\right), 0$. 


$$
\begin{aligned}
& \text { (1) } \Pi x\left(S x \rightarrow \mathcal{W}_{x} D x\right), 0\left[\mathrm{P}^{\prime}\right] \\
& \text { (2) } S c, 0\left[\mathrm{P}^{\prime}\right] \\
& \text { (3) } \mathcal{B}_{c} \square(D c \rightarrow H c), 0\left[\mathrm{P}^{\prime}\right] \\
& \text { (4) } \neg \mathcal{W}_{c} H c, 0 \text { [The 'negation' of } \mathrm{C} 4 \text { '] } \\
& \text { (5) } S c \rightarrow \mathcal{W}_{c} D c, 0[1, \Pi] \\
& \text { (6) } \neg S c, 0[5, \rightarrow] \\
& (8) *[2,6] \\
& \text { (7) } \mathcal{W}_{c} D c, 0[5, \rightarrow]
\end{aligned}
$$

The branch in the middle can be extended further. However, the branch to the right is open and complete. Hence, we can use this branch to read off a countermodel. This countermodel can then be used to verify that the argument is not valid in the class of all models.

The matrix of $\mathcal{B}_{c} \square(D c \rightarrow H c)$ is $\mathcal{B}_{x_{1}} \square\left(D x_{2} \rightarrow H x_{3}\right)$; the matrix of $\mathcal{W}_{c} H c$ is $\mathcal{W}_{x_{1}} H x_{2}$; and the matrix of $\mathcal{W}_{c} D c$ is $\mathcal{W}_{x_{1}} D x_{2}$.

$W=\left\{\omega_{0}\right\}, D=\{[c]\}, v(c)=[c]$, and the extensions of $D$ and $H$ are empty in $\omega_{0} . \mathfrak{R}, \mathfrak{A}$ and $\mathfrak{D}$ are empty. $v_{\omega_{0}}\left(\mathcal{B}_{x_{1}} \square\left(D x_{2} \rightarrow H x_{3}\right)\right)$ is the extension of $\mathcal{B}_{x_{1}} \square\left(D x_{2} \rightarrow H x_{3}\right)$ in $\omega_{0}, v_{\omega_{0}}\left(\mathcal{W}_{x_{1}} H x_{2}\right)$ is the extension of $\mathcal{W}_{x_{1}} H x_{2}$ in $\omega_{0}$, and $v_{\omega_{0}}\left(\mathcal{W}_{x_{1}} D x_{2}\right)$ is the extension of $\mathcal{W}_{x_{1}} D x_{2}$ in $\omega_{0}$.

$S c, 0$ is on the branch. Accordingly, $[c]$ is in the extension of $S$ in $\omega_{0}$ in $\mathcal{M}$. Hence, $S c$ is true in $\omega_{0}$ in $\mathcal{M} . \neg R c, 0$ is on the branch. Therefore, $[c]$ is not in the extension of $R$ in $\omega_{0}$ in $\mathcal{M}$, and so, $R c$ is false in $\omega_{0}$ in $\mathcal{M}$.

If $\neg R a_{m}, i$ is on the open branch $\mathcal{B}$ and $M$ is an n-place matrix with instantiations on $\mathcal{B}$ (where $x_{m}$ is the first free variable in $M$ and $a_{m}$ is the constant in $M\left[a_{1}, \ldots, a_{n} / x_{1}, \ldots, x_{n}\right]$ that replaces $\left.x_{m}\right)$, then $\left\langle\left[a_{1}\right], \ldots,\left[a_{n}\right]\right\rangle \in v_{\omega_{i}}(M)$ iff $M\left[a_{1}, \ldots, a_{n} / x_{1}, \ldots, x_{n}\right], i$ occurs on $\mathcal{B}$.

$\mathcal{B}_{x_{1}}\left(D x_{2} \rightarrow H x_{3}\right)\left[c, c, c / x_{1}, x_{2}, x_{3}\right], 0\left(=\mathcal{B}_{c}(D c \rightarrow H c), 0\right)$ is on the branch. $c$ is the constant in $\mathcal{B}_{x_{1}}\left(D x_{2} \rightarrow H x_{3}\right)\left[c, c, c / x_{1}, x_{2}, x_{3}\right]$ that replaces $x_{1}$ and $x_{1}$ is the first free variable in $\mathcal{B}_{x_{1}}\left(D x_{2} \rightarrow H x_{3}\right)$. Therefore, $\langle[c],[c],[c]\rangle$ is an element in $v_{\omega_{0}}\left(\mathcal{B}_{x_{1}}\left(D x_{2} \rightarrow H x_{3}\right)\right)$. If $R c$ is false in $\omega_{0}$ in $\mathcal{M}$, then $\mathcal{B}_{x_{1}}\left(D x_{2} \rightarrow\right.$ $\left.H x_{3}\right)\left[c, c, c / x_{1}, x_{2}, x_{3}\right]$ is true in $\omega_{0}$ in $\mathcal{M}$ iff $\langle v(c), v(c), v(c)\rangle$ is in $v_{\omega_{0}}\left(\mathcal{B}_{x_{1}}\left(D x_{2} \rightarrow\right.\right.$ $\left.\left.H x_{3}\right)\right)$. It follows that $\mathcal{B}_{x_{1}}\left(D x_{2} \rightarrow H x_{3}\right)\left[c, c, c / x_{1}, x_{2}, x_{3}\right]$ is true in $\omega_{0}$ in $\mathcal{M}$ iff $\langle v(c), v(c), v(c)\rangle$ is in $v_{\omega_{0}}\left(\mathcal{B}_{x_{1}}\left(D x_{2} \rightarrow H x_{3}\right)\right) . \quad\langle v(c), v(c), v(c)\rangle$ is in $v_{\omega_{0}}\left(\mathcal{B}_{x_{1}}\left(D x_{2} \rightarrow H x_{3}\right)\right)$. So, we conclude that $\mathcal{B}_{x_{1}}\left(D x_{2} \rightarrow H x_{3}\right)\left[c, c, c / x_{1}, x_{2}, x_{3}\right]$ is true in $\omega_{0}$ in $\mathcal{M}$. $\mathcal{B}_{x_{1}}\left(D x_{2} \rightarrow H x_{3}\right)\left[c, c, c / x_{1}, x_{2}, x_{3}\right]=\mathcal{B}_{c}(D c \rightarrow H c)$. It follows that $\mathcal{B}_{c}(D c \rightarrow H c)$ is true in $\omega_{0}$ in $\mathcal{M}$.

If $R c$ is false in $\omega_{0}$ in $\mathcal{M}$, then $\mathcal{W}_{x_{1}} H x_{2}\left[c, c / x_{1}, x_{2}\right]$ is true in $\omega_{0}$ in $\mathcal{M}$ iff $\langle v(c), v(c)\rangle$ is an element in $v_{\omega_{0}}\left(\mathcal{W}_{x_{1}} H x_{2}\right)$. Consequently, $\mathcal{W}_{x_{1}} H x_{2}\left[c, c / x_{1}, x_{2}\right]$ 
is true in $\omega_{0}$ in $\mathcal{M}$ iff $\langle v(c), v(c)\rangle \in v_{\omega_{0}}\left(\mathcal{W}_{x_{1}} H x_{2}\right)$. $\mathcal{W}_{x_{1}} H x_{2}\left[c, c / x_{1}, x_{2}\right], 0(=$ $\left.\mathcal{W}_{c} H c, 0\right)$ is not on the branch. $c$ is the constant in $\mathcal{W}_{x_{1}} H x_{2}\left[c, c / x_{1}, x_{2}\right]$ that replaces the first free variable in $\mathcal{W}_{x_{1}} H x_{2}$, namely $x_{1}$. Hence, $\langle[c],[c]\rangle$ is not an element in $v_{\omega_{0}}\left(\mathcal{W}_{x_{1}} D x_{2}\right)\left(v_{\omega_{0}}\left(\mathcal{W}_{x_{1}} D x_{2}\right)\right.$ is empty). Therefore, $\langle v(c), v(c)\rangle$ is not in $v_{\omega_{0}}\left(\mathcal{W}_{x_{1}} H x_{2}\right)$. So, it is not the case that $\mathcal{W}_{x_{1}} H x_{2}\left[c, c / x_{1}, x_{2}\right]$ is true in $\omega_{0}$ in $\mathcal{M}$. It follows that it is not the case that $\mathcal{W}_{c} H c$ is true in $\omega_{0}$ in $\mathcal{M}$, that is, $\mathcal{W}_{c} H c$ is false in $\omega_{0}$ in $\mathcal{M}$. For $\mathcal{W}_{x_{1}} H x_{2}\left[c, c / x_{1}, x_{2}\right]=\mathcal{W}_{c} H c$.

$\mathcal{W}_{x_{1}} D x_{2}\left[c, c / x_{1}, x_{2}\right], 0$ (that is, $\mathcal{W}_{c} D c, 0$ ) is on the branch. The first free variable in $\mathcal{W}_{x_{1}} D x_{2}$ is $x_{1}$ and $c$ is the constant in $\mathcal{W}_{x_{1}} D x_{2}\left[c, c / x_{1}, x_{2}\right]$ that replaces $x_{1}$. So, $\langle[c],[c]\rangle$ is an element in $v_{\omega_{0}}\left(\mathcal{W}_{x_{1}} D x_{2}\right)$. If $R c$ is false in $\omega_{0}$ in $\mathcal{M}$, then $\mathcal{W}_{x_{1}} D x_{2}\left[c, c / x_{1}, x_{2}\right]$ is true in $\omega_{0}$ in $\mathcal{M}$ iff $\langle v(c), v(c)\rangle \in v_{\omega_{0}}\left(\mathcal{W}_{x_{1}} D x_{2}\right)$. Hence, $\mathcal{W}_{x_{1}} D x_{2}\left[c, c / x_{1}, x_{2}\right]$ is true in $\omega_{0}$ in $\mathcal{M}$ iff $\langle v(c), v(c)\rangle \in v_{\omega_{0}}\left(\mathcal{W}_{x_{1}} D x_{2}\right) .\langle v(c), v(c)\rangle$ $\in v_{\omega_{0}}\left(\mathcal{W}_{x_{1}} D x_{2}\right)$. Consequently, $\mathcal{W}_{x_{1}} D x_{2}\left[c, c / x_{1}, x_{2}\right]$ is true in $\omega_{0}$ in $\mathcal{M}$. $\mathcal{W}_{x_{1}} D x_{2}\left[c, c / x_{1}, x_{2}\right]=\mathcal{W}_{c} D c$. In conclusion, $\mathcal{W}_{c} D c$ is true in $\omega_{0}$ in $\mathcal{M}$.

We have shown that $S c$ is true in $\omega_{0}$ in $\mathcal{M}$ and that $\mathcal{W}_{c} D c$ is true in $\omega_{0}$ in $\mathcal{M}$. Hence, $S c \rightarrow \mathcal{W}_{c} D c$ is true in $\omega_{0}$ in $\mathcal{M}$. Since $[c]$ is the only object in our domain, it follows that $\Pi x\left(S x \rightarrow \mathcal{W}_{x} D x\right)$ is true in $\omega_{0}$ in $\mathcal{M}$.

It follows that all the premises in The Doctoral Student Argument II are true in $\omega_{0}$ in $\mathcal{M}$, while the conclusion in this argument is false in $\omega_{0}$ in $\mathcal{M}$. Hence, the argument is invalid in the class of all models. Q.E.D.

Acknowledgements. The first version of this paper was finished in 2017. I would like to thank everyone who has read and commented on the text since then. Thanks also to Edwin Mares and an anonymous reviewer for The Australasian Journal of Logic for some short but helpful comments.

\section{References}

[1] Barcan (Marcus), R. C. (1946). A functional calculus of first order based on strict implication. Journal of Symbolic Logic 11, pp. 1-16.

[2] Bedke, M.S. (2009). The Iffiest Oughts: A Guise of Reasons Account of End-Given Conditionals. Ethics, Vol. 119, No. 4, pp. 672-698.

[3] Blackburn, P., de Rijke, M. and Venema, Y. (2001). Modal Logic. Cambridge University Press.

[4] Blackburn, P., van Benthem, J. and Wolter, F. (eds.). (2007). Handbook of Modal Logic. Elsevier.

[5] Broersen, J. M. (2011). Making a Start with the stit Logic Analysis of Intentional Action. Journal of Philosophical Logic, Vol. 40, No. 4, pp. 499-530.

[6] Broersen, J. M., Dastani, M. and van der Torre L. (2001). Resolving Conflicts between Beliefs, Obligations, Intentions, and Desires. In Salem Benferhat, Philippe Besnard (eds.) (2001). Symbolic and Quantitative Approaches to Reasoning with Uncertainty, Springer, pp. 568-579.

[7] Broome, J. (1999). Normative Requirements. Ratio (new series) XII 4, pp. 398-419. 
[8] Brunero, J. (2010). Self-Governance, Means-Ends Coherence, and Unalterable Ends. Ethics, Vol. 120, No. 3, pp. 579-591.

[9] Bull, R. A. (1969). On Modal Logic with Propositional Quantifiers. Journal of Symbolic Logic, Vol. 34, No. 2, pp. 257-263.

[10] Carnap, R. (1946). Modalities and Quantification. Journal of Symbolic Logic 11, 2, pp. 33-64.

[11] Chellas, B. F. (1980). Modal Logic: An Introduction. Cambridge: Cambridge University Press.

[12] Cohen, P. R. and Levesque, H. J. (1990). Intention is choice with commitment. Artificial Intelligence, 42, pp. 213-261.

[13] D’Agostino, M., Gabbay, D. M., Hähnle, R. and Posegga, J. (eds.) (1999). Handbook of Tableau Methods. Dordrecht, Kluwer Academic Publishers.

[14] Downie, R. S. (1984). The Hypothetical Imperative. Mind, New Series, Vol. 93, No. 372, pp. 481-490.

[15] Fagin, R. and Halpern, J. Y. (1988). Belief, Awareness and Limited Reasoning. Artificial Intelligence 34, pp. 39-76.

[16] Fagin, R., Halpern, J. Y., Moses, Y. and Vardi, M. Y. (1995). Reasoning About Knowledge. Cambridge, Mass., London, England: The MIT Press.

[17] Feldman, F. (1986). Doing the Best We Can: An Essay in Informal Deontic Logic. Dordrecht: D. Reidel Publishing Company.

[18] Feldman, F. (2004). Pleasure and the Good Life. Oxford/New York: Oxford University Press.

[19] Fine, K. (1970). Propositional quantifiers in modal logic. Theoria 36, pp. 336-346.

[20] Fine, K. (1980). First-Order Modal Theories. Studia Logica, Vol. 39, No. 2/3, pp. 159-202.

[21] Fitting, M. and Mendelsohn, R. L. (1998). First-Order Modal Logic. Kluwer Academic Publishers.

[22] Foot, P. (1972). Morality as a System of Hypothetical Imperatives. The Philosophical Review, Vol. 81, No. 3, pp. 305-316.

[23] Gabbay, D. M. (1976). Investigations in Modal and Tense Logics with Applications to Problems in Philosophy and Linguistics. Reidel, Dordrecht.

[24] Gallin, D. (1975). Intensional and Higher-Order Modal Logic: With Applications to Montague Semantics. Amsterdam: North-Holland.

[25] Garson, J. W. (1984). Quantification in Modal Logic. In D. M. Gabbay and F. Guenthner, (eds.) Handbook of Philosophical Logic 2, 1984, (2nd edition 3, 2001).

[26] Garson, J. W. (2006). Modal Logic for Philosophers. New York, Cambridge University Press.

[27] Gensler, H. J. (1985). Ethical Consistency Principles. The Philosophical Quarterly, Vol. 35, No. 139 , pp. $156-170$.

[28] Gensler, H. J. (2002). Introduction to Logic. London and New York: Routledge.

[29] Girle, R. (1998). Logical fiction: Real vs. ideal. In Hing-Yan Lee, Hiroshi Motoda (eds.) PRICAI98: Topics in Artificial Intelligence, pp. 542-552.

[30] Greenspan. P. S. (1975). Conditional Oughts and Hypothetical Imperatives. The Journal of Philosophy, Vol. 72, No. 10, pp. 259-276.

[31] Gochet, P. and Gillet, E. (1991). On Professor Weingartner's Contribution to Epistemic Logic. In G. Schurz and G. J. W. Dorn (eds.), Advances in Scientific Philosophy, Rodopi, pp. 97-115.

Australasian Journal of Logic (16:3) 2019, Article no. 3 
[32] Gochet, P. and Gribomont, P. (2006). Epistemic logic. In Dov M. Gabbay and John Woods, (eds.), Handbook of the History of Logic, vol. 7, North-Holland, pp. 99-195.

[33] Grover, D. L. (1972). Propositional Quantifiers. Journal of Philosophical Logic, Vol. 1, No. 2, pp. 111-136.

[34] Harsanyi, J. C. (1958). Ethics in Terms of Hypothetical Imperatives. Mind, Vol. 67, No. 267, pp. 305-316.

[35] Hill, Jr. T. E. (1973). The Hypothetical Imperative. The Philosophical Review, Vol. 82, No. 4, pp. $429-450$.

[36] Hill, Jr. T. E. (1989). Kant's Theory of Practical Reason. The Monist, Vol. 72, No. 3, Kant's Practical Philosophy, pp. 363-383.

[37] Hintikka, J. (1962). Knowledge and Belief: An Introduction to the Logic of the Two Notions. Cornell University Press. Ithaca (N. Y.). Reprinted 2005, King's College London Publications.

[38] Hocutt, M. (1972). Is epistemic logic possible? Notre Dame Journal of Formal Logic, XII, I (4), pp. 433-453.

[39] Hughes, G. E. and Cresswell, M. J. (1968). An Introduction to Modal Logic. London, Routledge.

[40] Jaspars, J. O. M. (1991). Fused Modal Logic and Inconsistent Belief. In M. De Glas and D. Gabbay (eds.), Proceedings of the 1st World Conference on the Fundamentals of Artificial Intelligence (WOCFAI'91), Paris, pp. 267-275.

[41] Jeffrey, R. C. (1967). Formal Logic: Its Scope and Limits. McGraw-Hill, New York.

[42] Kant, I. (1785). Grundlegung zur Metaphysik der Sitten. English translation in Paton (1948).

[43] Kaplan, D. (1970). S5 with quantifiable propositional variables. Journal of Symbolic Logic 35, p. 355.

[44] Korsgaard, C. M. (2008). The Normativity of Instrumental Reason. In Korsgaard (2008), The Constitution of Agency: Essays on Practical Reason and Moral Psychology. Oxford/New York: Oxford University Press.

[45] Kracht, M. (1999). Tools and Techniques in Modal Logic. Number 142 in Studies in Logic. Amsterdam, Elsevier.

[46] Kripke, S. A. (1959). A Completeness Theorem in Modal Logic. Journal of Symbolic Logic 24, pp. 1-14.

[47] Levesque, H. J. (1984). A Logic of Implicit and Explicit Belief. In Proceedings of the National Conference on Artificial Intelligence, pp. 198-202.

[48] Lewis C. I. and Langford, C. H. (1932). Symbolic Logic. New York, The Century Company.

[49] Lorini, E. and Herzig, A. (2008). A Logic of Intention and Attempt. Synthese, Vol. 163, No. 1, Knowledge, Rationality and Action, pp. 45-77.

[50] Marra, A. and Klein, D. (2015). Logic and Ethics: An Integrated Model for Norms, Intentions and Actions. In Wiebe van der Hoek, Wesley H. Holliday, Wen-fang Wang (eds.) (2015). International Workshop on Logic, Rationality and Interaction. Berlin-Heidelberg: Springer, pp. 268-281.

[51] Marshall, J. (1982). Hypothetical Imperatives. American Philosophical Quarterly, Vol. 19, No. 1, pp. 105-114.

[52] McLane, E. (1979). On the possibility of epistemic logic. Notre Dame Journal of Formal Logic, XX(3), pp. 559-574.

Australasian Journal of Logic (16:3) 2019, Article no. 3 
[53] Mele, A. R. (ed.). (2004). The Oxford Handbook of Rationality. Oxford: Oxford University Press.

[54] Meyer, J.-J. Ch. and van der Hoek, W. (1995). Epistemic Logic for AI and Computer Science. Cambridge University Press.

[55] Paton, H. J. (1948). The Moral Law: Kant's Groundwork of the Metaphysics of Morals. Translated and analysed by H. J. Paton. London and New York: Routledge (Reprinted 1991).

[56] Priest, G. (2005). Towards Non-Being. Oxford, Oxford University Press.

[57] Priest, G. (2008). An Introduction to Non-Classical Logic. Cambridge, Cambridge University Press.

[58] Rantala, V. (1982). Impossible World Semantics and Logical Omniscience. Acta Philosophica Fennica 35, pp. 106-115.

[59] Schroeder, M. (2004). The Scope of Instrumental Reason. Philosophical Perspectives, Vol. 18, Ethics, pp. 337-364.

[60] Schroeder, M. (2005). The Hypothetical Imperative? Australasian Journal of Philosophy 83, pp. 357-372.

[61] Schroeder, M. (2009). Means-End Coherence, Stringency, and Subjective Reasons. Philosophical Studies, Vol. 143, No. 2, pp. 223-248.

[62] Schroeder, M. (2015). Hypothetical Imperatives. In Mark Timmons and Robert N. Johnson (eds.). Reason, Value, and Respect: Kantian Themes from the Philosophy of Thomas E. Hill, $J r$., Chapter 4, Oxford University Press.

[63] Semmling, C., Wansing, H. (2008). From BDI and stit to bdi-stit logic. Logic and Logical Philosophy 17, 185-207.

[64] Shaver, R. (2006). Korsgaard on Hypothetical Imperatives. Philosophical Studies, Vol. 129, No. 2, pp. 335-347.

[65] Sim, K. M. (1997). Epistemic logic and logical omniscience: A survey. International Journal of Intelligent Systems, 12(1), pp. 57-81.

[66] Sim, K. M. (2000). Epistemic Logic and Logical Omniscience II: A Unifying Framework. International Journal of Intelligent Systems, Vol. 15, pp. 129-152.

[67] Smullyan, R. M. (1968). First-Order Logic. Heidelberg, Springer-Verlag.

[68] Sumner, L. W. (1996). Welfare, Happiness, and Ethics. Oxford: Clarendon Press.

[69] Sumner, L. W. (2000). Something in Between. In Roger Crisp and Brad Hooker (eds.), WellBeing and Morality: Essays in Honour of James Griffin, Oxford: Clarendon Press, pp. 1-19.

[70] Thijsse, E. (1992). Partial Logic and Knowledge Representation. PhD Thesis, Tilburg University.

[71] van Ditmarsch, H., van der Hoek, W., Kooi, B. (2008). Dynamic Epistemic Logic. Springer.

[72] van der Hoek, W. and Meyer, J.-J. Ch. (1989). Possible Logics for Belief. Logique et Analyse, 127-128, pp. 177-194.

[73] Wallace, R. J. (2001). Normativity, Commitment, and Instrumental Reason. Philosophers' Imprint, Volume 1, No. 3. pp. 1-26.

[74] Way, J. (2010). Defending the Wide-Scope Approach to Instrumental Reason. Philosophical Studies, Vol. 147, No. 2, pp. 213-233.

[75] Yap, A. (2014). Idealization, epistemic logic, and epistemology. Synthese, 191, pp. 3351-3366.

Australasian Journal of Logic (16:3) 2019, Article no. 3 